Portland State University

PDXScholar

Spring 1-1-2012

\title{
Coping with Interpersonal Conflicts at Work: An Examination of the Goodness of Fit Hypothesis Among Nurses
}

Robert Randon Wright

Portland State University

Follow this and additional works at: https://pdxscholar.library.pdx.edu/open_access_etds

Part of the Nursing Commons, Personality and Social Contexts Commons, and the Work, Economy and Organizations Commons

Let us know how access to this document benefits you.

\section{Recommended Citation}

Wright, Robert Randon, "Coping with Interpersonal Conflicts at Work: An Examination of the Goodness of Fit Hypothesis Among Nurses" (2012). Dissertations and Theses. Paper 610.

https://doi.org/10.15760/etd.610

This Dissertation is brought to you for free and open access. It has been accepted for inclusion in Dissertations and Theses by an authorized administrator of PDXScholar. Please contact us if we can make this document more accessible: pdxscholar@pdx.edu. 
Coping with Interpersonal Conflicts at Work: An Examination of the Goodness of Fit Hypothesis Among Nurses

by

Robert Randon Wright

A dissertation submitted in partial fulfillment

of the requirements for the degree of

Doctor of Philosophy

in

Applied Psychology

Dissertation Committee:

Cynthia D. Mohr, Chair

Leslie Hammer

Liu-Qin Yang

Robert Sinclair

Alan Cabelly

Portland State University

(C)2012 


\begin{abstract}
Increasingly, evidence indicates that workplace interpersonal conflicts (WIC) are the most upsetting/troublesome daily work stressors (Sulsky \& Smith, 2007), and within the context of nursing, WIC is a problem of high prevalence and intensity (Baltimore, 2006; Farrell, 1999). In relation to coping with stressors such as WIC, Lazarus and Folkman (1984) established the transactional model of stress and coping, where cognitive appraisals of the stressor (e.g., perceived control) are central to coping and classified all coping behaviors as either problem-focused or emotion-focused. They also proposed the "goodness of fit hypothesis", which predicts that problem-focused coping efforts used to cope with stressors of high appraised control and emotion-focused coping paired with stressors of low appraised control will produce the most effective outcomes. Contrary to these predictions, the general literature has produced inconsistent results, suggesting that context, research method, and individual difference variables (i.e., occupational tenure) should be considered when testing this hypothesis, particularly in novel contexts such as the nursing workplace.

This research was part of a larger study to identify key factors in the retention of nurses in the workforce, including a weekly survey spanning 12 weeks. Across the 12 week study period, 148 nurse participants completed an online survey, which included questions regarding the most negative interpersonal conflict at work for that week, the appraised controllability of the event, how the participant coped across 8 coping strategies, and how effective the coping efforts were. I used hierarchical linear modeling to test the goodness of fit hypothesis with these data, where the interaction terms between
\end{abstract}


coping frequency and control represented the key predictions of goodness of fit. Results revealed no support for the goodness of fit hypothesis, as the interactions were not significant. Consistent with goodness of fit, however, perceived control positively predicted problem-focused coping and negatively predicted emotion-focused coping for some nurses. This suggests that despite no improvement in coping outcomes, the underlying mechanisms for goodness of fit (i.e., matching perceived control with coping type) were in operation. Results also demonstrated no support of occupational tenure as a variable influential on the coping process. However, supplemental analyses revealed that as organizational tenure increased, nurses varied their coping strategies more, which then, in turn, produced more effective coping outcomes.

As the first effort to examine goodness of fit within the workplace to the best of my knowledge, these results suggest that the goodness of fit hypothesis may only have limited applicability to nursing, but should be examined in other nursing contexts and workplace conditions. Moreover, the length of time a nurse spends with an organization seems to influence one's coping style and the ability to match coping efforts with situational characteristics, producing more effective coping with interpersonal conflicts at work. These findings also imply that providing nurses with training about organizationspecifics may improve efforts to cope with interpersonal conflicts that arise in the workplace. 


\section{Dedication}

I would like to dedicate this dissertation to some very special people in my life. I would like to thank my parents, who have supported me through these 10 long years of post-high school education. Through it all, you have believed in me and helped me believe in myself. Although too young to know what he has done, I am grateful to my son, who was born in the midst of completing this project - he gave me another reason to finish. Finally, I would also like to thank my wife, Laura for standing beside me throughout this endeavor. Without your support, love and understanding, I could never have come this far; without you, this would have never happened. 


\section{Acknowledgements}

I would like to thank my committee for their assistance in this project. I would like to specifically thank Dr. Sinclair for giving me the opportunity to assist in his nursing research these past several years and his willingness to help me develop as a researcher in countless ways. I also would like to express my gratitude to Dr. Hammer, who has consistently been willing to serve on my committees and be my exemplar of what Occupational Health Psychology is all about. And, a special thanks to Dr. Yang, who has always extended insightful ideas on how to improve my research regardless of any circumstance or situation. I also acknowledge my indebtedness to Dr. Cabelly, whose wise discerning perspective has provided valuable insight into the process of completing a dissertation and becoming a doctorate. Finally, I want to extend my heartfelt thanks to my advisor, Dr. Cynthia Mohr. I am truly grateful for the many lessons you have taught me from both your words and your example. Even after all the years of my graduate experience, I still do not know how you balance everything and rise above to set the standard. Anyone who has you as a mentor is certainly one step ahead of everyone else.

This project was made possible through a grant [The Oregon Nurse Retention Project] from the Northwest Health Foundation and a grant from the National Institute of Occupational Safety and Health. I am grateful to the Oregon Nurse Retention Project team and to the Oregon Nurses Association (especially Dr. Sue Davidson), who played key roles in collecting these data. Finally, I would like to thank Dr. Ryan Olson and Dr. Brad Wipfli for believing I could finish this project and letting me work with them. 


\section{Table of Contents}

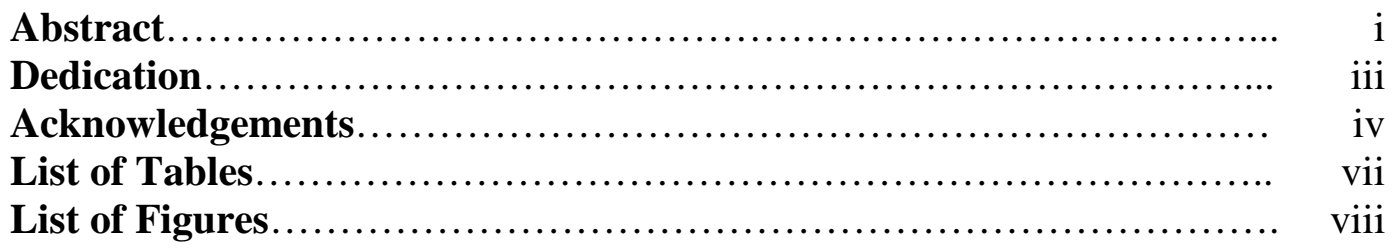

Chapter 1: Introduction.......................................... 1

Chapter 2: Workplace Interpersonal Conflict....................... 12

Definition of Workplace Interpersonal Conflict......................... 13

Outcomes of Workplace Interpersonal Conflict........................... 19

Chapter 3: The Transactional Model of Stress and Coping............ 27

Overview of Prominent Work Stress Models in the Literature............. 27

The Transactional Model of Stress and Coping........................ 33

Cognitive Appraisal............................................... 35

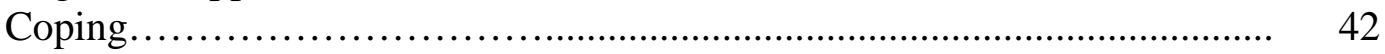

Chapter 4: The Goodness of fit Hypothesis........................ 61

An Appropriate Approach to Examining the Goodness of Fit Hypothesis... 63

Occupational Tenure.............................................. 72

Chapter 5: Study Purpose and Hypotheses............................ 82

Goodness of fit Hypothesis ........................................ 83

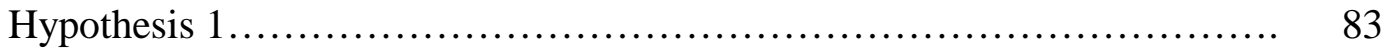

Occupational Tenure as Moderator of Goodness of fit Hypothesis.......... 84

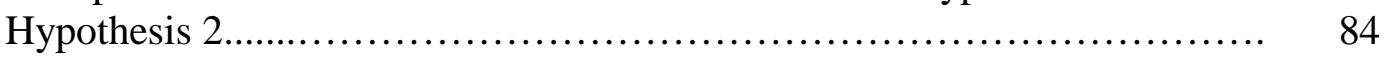

Occupational Tenure Main Effect on Coping Frequency................. 84

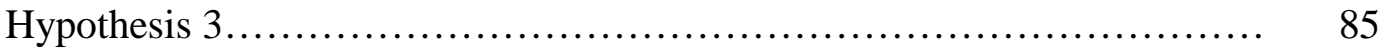

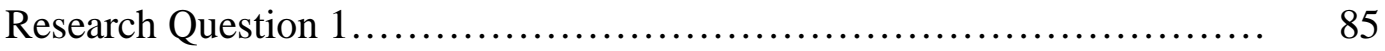

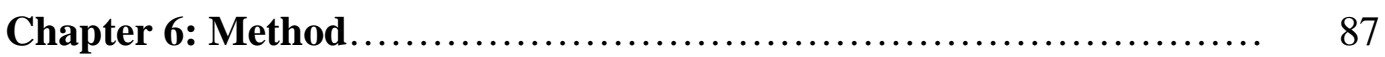

Participants.................................................... 89

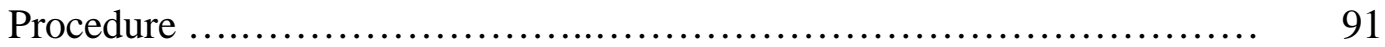

Measures.......................................................... 93

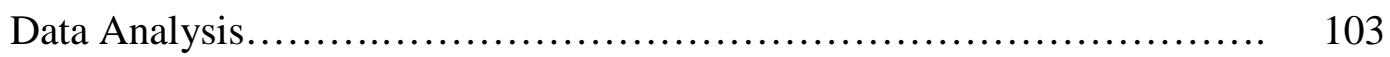

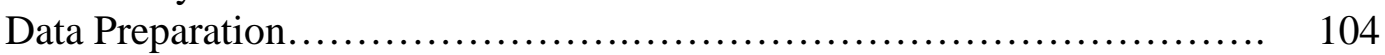

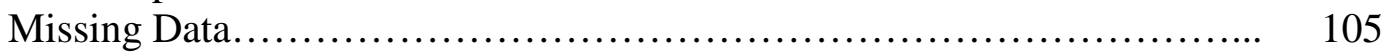

Hypothesis Testing Analyses....................................... 108

Research Question Analysis.......................................... 111 
Chapter 7: Results............................................. 112

Descriptive Information.............................................. 112

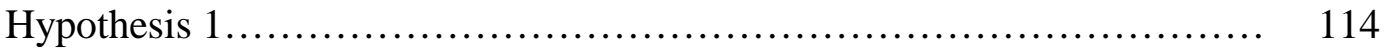

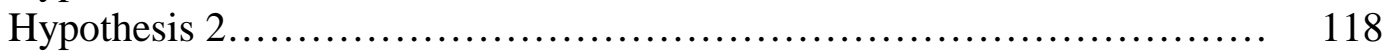

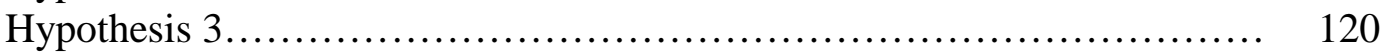

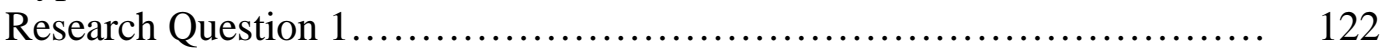

Supplemental Analyses............................................ 124

Chapter 8: Discussion................................................... 131

The Goodness of Fit Hypothesis...................................... 132

Occupational Tenure and Coping..................................... 139

Coping Frequency Variation ............................................... 143

Other Findings....................................................... 148

Theoretical Implications......................................... 150

Practical Implications............................................ 152

Potential Limitations.................................................. 156

Strengths and Future Research...................................... 160

Conclusion ......................................................... 165

References.............................................................. 181

Appendix A: Glossary of Acronyms................................. 200

Appendix B: The Oregon Nurse Retention Project Demographics and

Weekly Survey .................................................... 201 


\section{List of Tables}

Table 1: Comparison of Model Fit across 2-factor and 3-factor Model

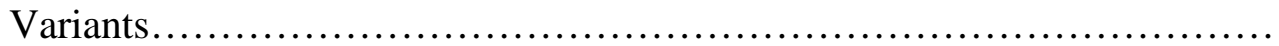

Table 2: Means and Standard Deviations of the Individual Weekly Coping

Frequency and Coping Effectiveness Variables

Table 3: Complete Correlation Matrix for Demographic and Study

Variables across Level 1 and Level $2 \ldots \ldots \ldots \ldots \ldots . .168$

Table 4: Hypothesis 1 Multilevel Regression Results Predicting Weekly

Coping Effectiveness from Weekly Control and Coping........................

Table 5: Hypothesis 2 Multilevel Regression Results Predicting Weekly

Coping Effectiveness from Control, Coping, and Occupational Tenure ......

Table 6: Hypothesis 3 Multilevel Regression Results of Variables

Predicting Problem- and Emotion-Focused Coping Frequency as the Only

Predictor in the Model...........................................................

Table 7: Research Question 1, Univariate Regression Results for Occupational and Organizational Tenure Predicting Person Coping Variation 


\section{List of Figures}

Figure 1: A Model of Workplace Interpersonal Conflict................. 173

Figure 2: Venn Diagram of the Interpersonal Conflict Construct............ 174

Figure 3: Transactional Model of Stress and Coping.................... 175

Figure 4: The Goodness of Fit Hypothesis (Lazarus \& Folkman, 1984)..... 176

Figure 5: The Goodness of fit Hypothesis Testing Model.................. 177

Figure 6: The Goodness of fit Hypothesis Testing Model Moderated by

Occupational Tenure............................................ 178

Figure 7: Occupational Tenure Main Effects on Coping Frequency......... 179

Figure 8: The Oregon Nurse Retention Project Research Design........... 180 


\section{Chapter 1: Introduction}

Each year, work stress is responsible for an estimated $\$ 150$ to $\$ 300$ billion loss within organizations across the nation (Ball, 2004; de Carteret, 1994; Matteson \& Ivancevich, 1987). Contributing to this alarming statistic, there are numerous detrimental organizational outcomes associated with high work stress such as employee turnover (e.g., Quick, Quick, Nelson, \& Hurrell, 1997; Shirom, 2003), lower productivity (e.g., Colligan \& Higgins, 2005; Tetrick \& Quick, 2011), excessive absenteeism (e.g., Hackett \& Bycio, 1996; Hardy, Woods, \& Wall, 2003), and increased workplace violence (e.g., Barling, Dupre \& Kelloway, 2009). Moreover, work stress is a substantial contributor to increases in employee health problems including cardiovascular disease (e.g., Kivimaki et al., 2006; Schwartz, Pickering, \& Landsbergis, 1996), musculoskeletal disorders and injuries (e.g., Johnston, 2007), as well as immunosuppression (e.g., Cohen, Miller, \& Rabin, 2001). In fact, some have estimated that nearly $80 \%$ of all illness is stress-related, making work stress a serious health concern (Pretrus \& Kleiner, 2003).

Increasingly, evidence indicates that interpersonal conflicts, in particular, are substantially contributing to this problem as they are frequently the most upsetting and troublesome of daily stressors (e.g., Beach, Martin, Blum, \& Roman, 1993; Bolger, DeLongis, Kessler, \& Schilling, 1989; Farrell, 1999; Keenan \& Newton, 1985; Leiter, 2005; Smith \& Sulsky, 1995). Similarly, employees consistently rank interpersonal conflict as the most stressful and troubling events at work (e.g., Beach et al., 1993; Farrell, 1999; Keenan \& Newton, 1985; Leiter, 2005; Smith \& Sulsky, 1995). Indeed, in a nationally representative survey of the Canadian workforce, interpersonal conflicts at 
work were the third most frequently cited source of work-related stress (Williams, 2003). Other recent estimates suggest that interpersonal conflicts at work occur on at least $50 \%$ of work days (Hahn, 2000) and negatively impact more than $26 \%$ of the entire worker population (de Raeve, Jansen, van den Brandt, Vasse, \& Kant, 2009). Furthermore, workplace interpersonal conflict (WIC) has been specifically identified as a significant precursor to the development of severe social problems such as workplace aggression and violence (Barling et al., 2009; Goldstein, 1994; Pearson, Andersson, \& Wegner, 2001), and depression and withdrawal (Frone, 2000; Spector \& Jex, 1998). In fact, interpersonal conflict may be the most important workplace stressor for organizations to address because some estimates suggest that this stressor represents one of the largest reducible costs to any organization (Dana, 1999; Keenan \& Newton, 1985).

Building off of the social psychological and organizational literatures, Barki and Hartwick (2004) defined WIC as a dynamic process between interdependent parties that involves perceived disagreements, interference with others' goal-oriented behavior, and negative emotion. Under this definition, WIC encompasses a broad range of behaviors from daily disagreements between individuals to more intense behaviors such as sabotage (e.g., Dunn, 2003) or even verbal and physical intimidation (Spector \& Jex, 1998). Outcomes associated with WIC are similarly diverse, often categorized as outcomes that either primarily affect the organization (e.g., employee turnover, productivity) or the employee/individual (e.g., physical health, mental health). Within the specific context of nursing, WIC has become a substantial problem as conflict can develop among multiple sources (e.g., nurse managers, physicians, coworkers, patients/families), contributing to 
an observed overall high incidence, prevalence and intensity of WIC among nurses (e.g., Baltimore, 2006; Cheuk, Wong, Swearse, \& Rosen, 1997; Dewe, 1987; Dunn, 2003; Farrell, 1999; 2001; Leiper, 2005). This is an important issue in healthcare, as nurses are becoming increasingly more reliant upon teams of nurses and other health professionals to deliver quality patient care, making interpersonal interactions key to successful patient recovery and rehabilitation (Havens et al., 2010; Makinen et al., 2003). Indeed, nurse WIC remains a significant problem that affects both nurses and the work they do, requiring further empirical attention (Almost, 2006; Dunn, 2003).

Due to the pervasive nature of WIC, most, if not all, employees will be faced with the dilemma of coping with stressful conflicts at work at one point or another. Although many work stress models and theories could be used to explain causes, outcomes or the progression of WIC (e.g., Bakker \& Demerouti, 2007; Karasek, 1979; Siegrist, 1996), the transactional model of stress and coping (Lazarus \& Folkman, 1984) seems to offer the most promise in addressing coping with WIC. Indeed, unlike other work stress models, the transactional model offers the primary advantage of explaining the entire stress and coping process according to subjective evaluations of the individual. Moreover, the transactional model provides a unique comprehensive framework of coping responses that are predicted to be most effective under certain circumstances (Lazarus, 1993; Lazarus \& Folkman, 1984). Therefore, as the transactional model offers the best approach to examining coping with WIC, I used this model to examine the effectiveness of different coping strategies in relation to WIC among nurses. 
According to the transactional model, coping is depicted as an outcome of subjective evaluations of the stressor known as cognitive appraisal. Cognitive appraisal unfolds in three interrelated processes known as primary appraisal, secondary appraisal, and reappraisal (Lazarus \& Folkman, 1984). Specifically, during primary appraisal, the individual determines whether the encounter is benign/positive, irrelevant, or potentially threatening or harmful to the individual. Then, under secondary appraisal, the individual proceeds to evaluate the amount of control the individual possesses over the stressor and related outcomes as well as consider potential coping efforts. Finally, based upon additional information from the environment (e.g., stressor has changed, coping efforts were not effective), the individual may engage in reappraisal, which may alter the original primary or secondary appraisal. As such, when an individual is exposed to a potential stressor, such as WIC, the individual must determine if the conflict is stressful, the amount of control the individual has over the conflict and related outcomes, and, if applicable, consider altering the original appraisal of the conflict before engaging a coping strategy.

Under the transactional model, coping is considered to be constantly changing cognitive or behavioral efforts employed by an individual to manage specific demands that are appraised as stressful (Lazarus \& Folkman, 1984). This process approach to coping uses situational characteristics and cognitive appraisals to explain both betweenand within-person fluctuations in coping, thus offering the ability to account for changes in coping beyond what average levels of coping would indicate (e.g., Tennen \& Affleck, 1996). Moreover, under this definition, virtually any behavior used to deal with stress 
could be considered coping (Lazaurs \& Folkman, 1984), making it essential to have a classification of coping behaviors. Although many models and classifications of coping behaviors have been proposed (e.g., control/escape coping, Latack, 1986; task/emotion/avoidance coping, Endler \& Parker, 1990), the transactional model's dichotomy of problem-focused and emotion-focused coping remains the most comprehensive and well-supported in the literature (e.g., Zakowski, Hall, Klein, \& Baum, 2001). Whereas problem-focused coping is primarily concerned with altering or managing the stressor or problem itself, emotion-focused coping is behavior directed towards emotional regulation, including avoidance behaviors (Lazarus \& Folkman, 1984). This distinction has been investigated and supported across numerous studies (e.g., Folkman \& Lazarus, 1985; Tennen, Affleck, Armeli, \& Carney, 2000; Zeidner \& Saklofske, 1996), including in studies of nurses (Lambert, Lambert \& Ito, 2004; Xianyu \& Lambert, 2006).

Of additional importance, the literature defines effective or adaptive coping as coping behavior that produces beneficial outcomes for the individual in relation to the stressor (Zeidner \& Saklofske, 1996). On the other hand, ineffective or maladaptive coping is considered to have the opposite effect, eliciting detrimental or non-beneficial outcomes for the individual. Moreover, several studies have suggested that coping effectiveness may best be evaluated from the perspective of the individual (e.g., Aldwin \& Revenson, 1987; Dewe, 1989). Consistent with the transactional model, this type of evaluation permits any type of coping to be considered for potential effectiveness. However, consistent with the literature, substance use coping is not effective, which has 
been identified as maladaptive coping (e.g., Frone, 2008; Hyman \& Sinha, 2008; Mohr et al., 2001), including among nurses (Trinkoff, Zhou, Storr, \& Soeken, 2000).

Based upon the transactional model, optimal coping effectiveness should occur when there is an appropriate match between control appraisals (i.e., high, low) and coping efforts (i.e., problem-focused, emotion-focused). Known as the "goodness of fit" hypothesis (Lazarus \& Folkman, 1984), problem-focused coping efforts used to cope with stressors of high appraised control and emotion-focused coping matched to stressors of low appraised control are predicted to produce the most effective coping outcomes. For example, in a WIC where a nurse makes a high control appraisal, a problem-focused coping strategy (e.g., direct confrontation) would likely be more effective at resolving the conflict (i.e., adaptive outcome); whereas, in a WIC with a low control appraisal, an emotion-focused coping strategy (e.g., emotional social support) should reduce the negative emotional response (i.e., adaptive outcome). Hence, this hypothesis assumes that the match between control appraisal and coping strategy ultimately determines coping effectiveness.

Despite the intuitiveness of these predictions, the general literature investigating this hypothesis has produced inconsistent results, highlighting the important role both context and research method play in providing an appropriate test of this hypothesis. Although the goodness of fit hypothesis has not been tested within the workplace or among nurses, there is some evidence supportive of these predictions within this context (e.g., Hahn, 2000; Portello \& Long, 2001). Moreover, the literature suggests two specific methodological characteristics that may improve the ability to conduct an appropriate test 
of goodness of fit. First, many of the studies failing to support the goodness of fit hypothesis have focused on physical health stressors, suffering from little variation in perceptions of control (e.g., Kendall \& Terry, 2008); whereas those studies finding support have focused on stressors which are more likely to fluctuate in perceived control both within- and between-persons (e.g., social stressors; e.g., Compas, Malcarne, \& Fondacaro, 1988; Forsythe \& Compas, 1987). Second, rather than relying solely upon averages across the entire sample (between-persons), studies finding the most support for the goodness of fit hypothesis have examined multiple stressors (per person) over time using a within-person approach (Park, Armeli, \& Tennen, 2004; Park, Folkman, \& Bostrom, 2001). Therefore, consistent with this literature, I evaluated longitudinal coping effectiveness data in relation to nurse WIC using a within-persons approach and thus expected to find confirming evidence of the goodness of fit hypothesis.

Results from these studies employing a within-person approach, also suggest that individual differences may influence the predictions of the goodness of fit hypothesis (Park et al., 2004). Within the nursing workplace, occupational tenure may be one of these variables. Indeed, the literature suggests that as tenure increases, employees are often more attentive to particular stressor cues in the environment, thus likely amplifying the effect of the match between control appraisal and coping effort for those with greater tenure (Bradley, 2007; Cortina \& Wasti, 2005; Moser \& Galais, 2007). Additionally, the literature has uncovered an association between occupational tenure and coping frequency, in that tenure is generally positively associated with problem-focused and negatively related to emotion-focused coping (e.g., Havlovic \& Keenan, 1995; Lambert, 
Lambert \& Yamase, 2003). Virtually all of the limited literature examining occupational tenure and coping, however, has relied upon cross-sectional designs employing a between-persons approach. Indeed, the literature has yet to examine the effect of occupational tenure as a moderator of goodness of fit or coping frequency using a withinpersons perspective among nurses, which could substantially contribute to the literature on tenure and coping. Consistent with the literature, I expected to find evidence of a moderating effect of occupational tenure on the goodness of fit hypothesis as well as a main effect of occupational tenure on coping frequency.

In sum, I examined the goodness of fit hypothesis among a nursing sample where coping effectiveness is modeled as a function of fit between problem-focused/emotionfocused coping and perceived control. Consistent with the literature's suggestions for an appropriate test of goodness of fit, I examined longitudinal data of coping with multiple social stressors (i.e., WIC) using a within-persons approach. Furthermore, I investigated occupational tenure as a potential moderating variable of this relationship, and as a predictor of coping frequency. Thereby, this dissertation was an in-depth investigation of the goodness of fit hypothesis and the relationship between occupational tenure and coping for nurses who are dealing with WIC.

\section{Contributions of the Present Study}

Although the goodness of fit hypothesis has been examined within several contexts, the literature continues to lack a test of the goodness of fit hypothesis within the workplace context and, more specifically, among nurses. As such, the goal of this dissertation was to apply the theoretical propositions of the goodness of fit hypothesis 
within the nursing workplace context in relation to workplace interpersonal conflict using a within-persons approach. As far as I am aware, this dissertation offers the unique contribution of providing the first examination of the goodness of fit hypothesis in the nursing workplace context relative to significant social stressors. Moreover, this dissertation also contributes to the limited literature on occupational tenure and coping by providing a within-persons investigation of occupational tenure as a potential moderator of the goodness of fit hypothesis and as a predictor of coping frequency among a nursing sample. Therefore, this dissertation presents unique contributions to the literature by investigating the goodness of fit hypothesis in the nursing workplace and the role that occupational tenure plays in effectively coping with WIC using a within-persons approach.

This dissertation presents additional practical contributions. Perhaps most importantly, nurses are likely to benefit considerably from the results of this study as the literature has identified WIC as an area of critical importance to nursing (Almost, 2007; Dunn, 2003). Indeed, despite the likely effectiveness of prevention strategies implemented to decrease interpersonal conflict (e.g., policies, sanctions), it is virtually certain that nurses (and other employees) will be exposed to interpersonal conflicts at some point while at work. Organizations can promote more effective coping by offering specific training to managers that encourage more effective coping and implement policies such as more diversity in nursing teams to improve coping outcomes. Although organizational interventions can be effectively implemented to address and prevent WIC, potential conflict is universal within all organizations, especially for those with frequent 
interactions with other employees and/or the public. Therefore, in anticipation that preventative efforts cannot avert all conflict, organizations can potentially positively affect every nurse by empowering nurses, who interact with both employees and the public, with knowledge of how to effectively cope with WIC (see Practical Implications in Chapter 8). Moreover, this information can be disseminated through training sessions in nursing and in occupations prone to high rates of WIC. Thus, this study offers strong contributions in both theoretically- and practically-important dimensions.

\section{Present Study}

This research was part of a larger project, the Oregon Nurse Retention Project, which was conducted collaboratively between Portland State University and the Oregon Nurses Association to identify factors that lead to retention of nurses in their current employment and in the nursing workforce. This project was supported by funding from the Northwest Health Foundation to Robert Sinclair, Ph.D. and Cynthia Mohr, Ph.D as well as a grant from the National Institute of Occupational Safety and Health. I have been involved in all aspects of the project from designing the study to data analysis and dissemination (see Chapter 6). The 148 eligible participants in this study agreed to take Internet-based surveys once a week for up to 12 weeks, providing details regarding their most negative/stressful interpersonal conflict for that week, appraisals of control, how they coped with it, and their perception of how effective each coping strategy was. Moreover, weekly data were hierarchically structured, with each participant potentially reporting 12 weekly interpersonal conflicts and related coping behaviors. As such, this type of data permitted a within-persons examination and required sophisticated analytic 
techniques due to possible missing observations (i.e., data are unbalanced). Therefore, I used Hierarchical Linear Modeling (HLM, v6.0; Raundenbush, Brik, Cheong, \& Congdon, 2000), which can uniquely examine between- and within-persons effects while handling missing data.

In the following chapters, I will briefly present a model of workplace interpersonal conflict while reviewing the definition of workplace interpersonal conflict and highlighting many of the outcomes associated with WIC, paying particular attention to the effects of WIC in nursing. Next, I will elaborate the transactional model of stress and coping (Lazarus \& Folkman, 1984) and briefly detail the nursing literature in this area. Subsequently, I will examine the evidence supporting the goodness of fit hypothesis and ways in which occupational tenure may be influential in the coping process for nurses. Following which, I will discuss the purpose of this dissertation and present my hypotheses. Then, I will describe my research methods and results of my analyses. Finally, I will conclude with a discussion of the results and associated contributions and limitations of this study. 


\section{Chapter 2: Interpersonal Conflict in the Workplace}

A large body of research demonstrates that negative interpersonal interactions have a pervasive detrimental effect on well-being and social functioning (e.g., Bolger et al., 1989; Mohr et al., 2003; Newsom, Nishishiba, Morgan, \& Rook, 2003; Nezlek \& Plesko, 2003; Ruehlman \& Karoly, 1991), especially in the workplace context (e.g., Bruk-Lee \& Spector, 2006; Cortina, Magley, Williams, \& Langhout, 2001; Frone, 2000). Interpersonal stressors such as conflicts, are often associated with such negative outcomes as depression and withdrawal (e.g., Bruk-Lee \& Spector, 2006; Frone, 2000; Rook, 2001; Ruehlman \& Karoly, 1991), negative affect and mood (e.g., Beach et al., 1993; Bolger et al., 1989; Mohr et al., 2003), poor physical health (e.g., de Raeve et al., 2009), and are even predictive of future work disability (Appelberg, Romanov, Heikkila, Honkasalo, \& Koskenvuo, 1996) and psychiatric problems (Romanov, Appelberg, Honkasalo, \& Koskenvuo, 1996). Even more disconcerting, interpersonal conflicts are a common issue for people, spanning cultures, age, and contexts (Larson \& Richards, 1994; Valsiner \& Cairns, 1992). Indeed, exposure to conflict begins as early as childhood and adolescence (e.g., Jensen-Campbell \& Graziano, 2000) and can become a frequent stressor for adults in the workplace (e.g., Frone, 2000; Hahn, 2000; Spector \& Jex, 1998).

Across occupations, interpersonal conflicts in the workplace are consistently cited as the most stressful events for employees (e.g., Beach et al., 1993; Farrell, 1999; Leiter, 2005; Smith \& Sulsky, 1995). Moreover, the workplace is a unique context wherein interpersonal conflict can produce negative outcomes for both employee and organization, raising concern for the health and well-being of both (e.g., Bennett, Cook, 
\& Pelletier, 2003). Additionally, interpersonal conflicts at work occur regularly (de Raeve et al., 2009; Hahn, 2000); recent estimates suggest that conflicts, especially within the nursing profession, are escalating in both rate of occurrence and intensity (e.g., Almost, 2006; Baltimore, 2006; Farrell, 1999).

Effectively handling or coping with WIC is another concern, as those who do not effectively cope with WIC report increased levels of distress (e.g., Portello \& Long, 2001), high levels of subjective anger (Hahn, 2000), and often become victims of workplace bullying or sexual harassment (e.g., Cortina \& Wasti, 2005; Zapf \& Gross, 2001). This is a chronic issue for nurses, as the collaborative health care context requires the nurse to deal or cope with conflicts that arise and, if handled ineffectively, such outcomes as patient care or team productivity may suffer (e.g., Duddle \& Boughton, 2007; Farrell, 1999). As this dissertation is focused on how nurses cope with WIC, I discuss a model of WIC (see Figure 1), including the definition of WIC and the detrimental outcomes associated with WIC, and conclude with a brief outline of how WIC affects nursing.

\section{Definition of Workplace Interpersonal Conflict}

The social psychological literature contains several influential theories that have guided the development of a formal definition of WIC. Within this literature, interpersonal conflict has been conceptualized as being composed of certain properties. Specifically, cognitions, situational interdependence, conflicting behavior and negative emotion have been proposed as properties of interpersonal conflict (and later for WIC), each of which I discuss briefly below. 
Cognitions. As one of the first properties to be considered, cognition, usually in the form of disagreement, was often theorized to be central to conflict. Social Comparison Theory (Festinger, 1954), for example, postulates that interpersonal conflict naturally occurs as individuals have differing opinions that are realized through comparing one's own cognitions to that of others within the social context. For example, a conflict may arise between two nurses as they realize they disagree regarding how much time should be spent focused on the patient versus administrative issues. As such, this theory introduces cognitive comparisons as a key mechanism for interpersonal conflict. As a logical extension to this, Kelley and Thibaut (1978) developed Social Exchange Theory, where social interactions are depicted as a function of each party's desired outcome, similar to an economic exchange model. Integral to this theory, individuals cognitively assess situations and interactions for their likely rewards and costs. Therefore, using the above example, one nurse may be inclined to alter his/her own opinion rather than continue in the disagreement based upon the likely minimal reward and high cost of the interaction. Therefore, these theories advocate the important role cognitions have in social interactions and suggest that interpersonal conflicts are often comprised of disagreement.

Situational interdependence. Social Exchange Theory and other research highlight the important role of situational interdependence between parties in conflict. Indeed, interdependence, or the reliance on others for important personal outcomes, is considered an important predictor of behavior in Social Exchange Theory (Kelley \& Thiabut, 1978), where strong interdependence elicits more cooperation and weak 
interdependence elicits less. For example, when people are dependent upon each other for important outcomes (e.g., providing quality patient care), consideration of long-term consequences (e.g., future interactions, patient's health) rather than just short-term rewards in conflict may become increasingly important (Clark \& Grote, 1998; Walster, Walster, \& Berscheid, 1978). In a similar line of research, Deutsch (1949) dichotomized interdependence as "positive" (i.e., cooperation) or "negative" (i.e., competition) and found that interpersonal conflict was more likely to occur when parties were pitted against one another for some recognition/honor, resource, or reward (e.g., winning a game, obtaining a prize). Consequently, interpersonal conflict can be understood as a function of competition between individuals who depend upon one another for some desired outcome. As such, interpersonal conflict should occur under conditions of situational interdependence.

Conflicting behavior. Conflicting behaviors of parties involved in interpersonal conflict have been proposed as a third property. Spearheading this proposition, Deutsch (1962) demonstrated that conflicts between parties were often comprised of behaviors that impeded the others' goal-directed behavior. Indeed, in later publications, Deutsch used behavioral goal interference as the defining aspect of interpersonal conflict (Deutsch, 1973; 1990) and subsequent research has noted how having one's goals blocked or impeded can produce conflict (e.g., Ruehlman \& Wolchick, 1988). Along a similar note, Muzafer Sherif conducted a series of studies regarding group conflict and cooperation in the formulation of another theoretical perspective, Realistic Conflict Theory (Sherif, Harvey, White, Hood, \& Sherif, 1961). According to this theory, conflict 
erupts between groups or individuals as they compete for limited resources that are highly valued by each party. Conflict is thus conceptualized as the natural outcome of the inability of all individuals to gain important or even vital limited resources to achieve their goals. For example, a nurse on a prior shift may intentionally or unintentionally not complete important paperwork necessary for the next nurse on shift to provide adequate patient care and thus prevent this nurse from providing this care. As such, behavior that interferes with the goals of others is another property of interpersonal conflict.

Negative emotion. Finally, the literature supports negative emotion as an additional property of interpersonal conflict. Indeed, proposed as an explanation for aggressive behavior under the Stimulus-Response behavioral paradigm (Jones, 1998), the frustration-aggression hypothesis (Dollard, Doob, Mowrer, Miller, \& Sears, 1939) depicts frustration leading to aggression and aggression to frustration in a spiral of negative emotion and behavior. Moreover, in connection with the frustration-aggression hypothesis, negative affect (e.g., frustration, anger), in particular, has been proposed as a major component of aggression and conflict (e.g., Berkowitz, 1988; Zillman, 1994). Based upon these theoretical advances, later empirical investigation has demonstrated negative emotions (e.g., fear, jealousy, anxiety, frustration) are present during most interpersonal conflicts, especially those that are the most important and the most stressful to individuals (e.g., Jehn, 1995; 1997). The view that emotion is not merely an outcome of conflict, but part of conflict itself is further supported by Zajonc (1980), who argued that cognitions typically incorporate affective states and should be regarded as an important independent system (e.g., emotions do not solely rely upon cognitions). For 
example, a conflict may ensue between a nurse and physician, simply based upon the nurse's fear or anxiety regarding the attitude or demeanor of that physician. Therefore, the literature also supports the inclusion of negative emotion as a property of interpersonal conflict.

WIC defined. The organizational literature has produced substantial support for each of these properties of interpersonal conflict within the specific context of the workplace (Pondy, 1967; Putnam \& Poole, 1987; Thomas, 1992). In a comprehensive review of this literature, Barki and Hartwick (2004) developed a definition of interpersonal conflict. Specifically, they defined interpersonal conflict in organizational workplace settings as "a dynamic process that occurs between interdependent parties as they experience negative emotional reactions to perceived disagreements and interference with the attainment of their goals" (p. 234). This definition allows for dynamic interplay between disagreement, interference, and negative emotions such that one property can, but does not always, lead to another or vice versa in a continuous interactive cycle between the properties (Barki \& Hartwick, 2004). Therefore, the cognition of disagreement, the behavior of interference with goals, and negative emotion are all considered properties of interpersonal conflict. As depicted in the Venn diagram in Figure 2, interpersonal conflict can be defined as the presence of one or a combination of any of these properties. It is important to note that Barki and Hartwick (2004) describe this as a general definition of interpersonal conflict; however, their definition was developed according to a literature review consisting exclusively of studies using workplace samples. Furthermore, this definition is consistent with, and incorporates, 
other definitions of WIC including the occupational health psychology literature (e.g., Lee, 2007; Spector \& Jex, 1998) and recent research within the organizational literature (e.g., de Raeve et al., 2009; Schieman \& Reid, 2008). Therefore, Barki and Hartwick's (2004) definition is representative of WIC and is used in the model of WIC (see Figure 1) for this dissertation.

One modification made to the WIC definition in comparison to that of general interpersonal conflict, however, is the unique function Barki and Hartwick (2004) assign to interdependence. Based off of the findings of an earlier workplace study on conflict (Barki \& Hartwick, 2001), interdependence is conceptualized not as a specific component of interpersonal conflict per se, but as a structural antecedent, setting the stage for conflict to potentially occur. Although this conclusion seems contrary to the social psychological literature (e.g., Kelley \& Thibaut, 1978), Barki and Hartwick (2004) still maintain that interdependence plays a critical role in WIC as a necessary antecedent, not as an actual property of the conflict itself. In other words, without a certain amount of interdependence between parties at work, WIC could not develop. To be consistent with the definition of WIC provided by Barki and Hartwick (2004), I considered interdependence as a structural precursor of conflict at work in this dissertation and assume a certain degree of interdependence in any instance of WIC.

Finally, it is important to note that the definition of WIC provided by Barki and Hartwick (2004) highlight the subjective nature of WIC. Indeed, Barki and Hartwick (2004) acknowledge that both the presence and the intensity of any given instance of WIC will be determined by an individual's subjective perception of the three properties. 
Indeed, the individual's perception is vital in order for conflict to exist, regardless of the objective characteristics of the situation (see Figure 1). Therefore, interpersonal conflict, as defined by Barki and Hartwick (2004), varies substantially in occurrence and intensity between individuals and even across situations (e.g., within individuals). For the purpose of coping with WIC stressors, it is important to note that this is consistent with the subjective nature of coping, making WIC an appropriate stressor to examine under the transactional model of stress and coping. I will return to this subject in Chapter 3.

Therefore, according to this definition, WIC encompasses a large range of workplace behaviors and a corresponding diverse set of outcomes are also associated with WIC. As such, I now briefly discuss two categories of WIC outcomes and how these relate to nursing.

\section{Outcomes of Workplace Interpersonal Conflict}

As a social stressor, WIC has been linked to several deleterious outcomes. The literature has grouped these outcomes into two broad categories: those that primarily affect the organization and those that primarily affect the employee (e.g., Bruk-Lee \& Spector, 2006; Frone, 2000; Wall \& Callister, 1995). Therefore, using this dichotomy as a framework, I now discuss the outcomes of WIC that have been identified within the literature (see Figure 1).

Organizational outcomes. As organizations increasingly rely more upon work teams (e.g., Sparrowe \& Liden, 1997; 2005; Tse, Dasborough, \& Ashkanasy, 2008) and

focus on work productivity (Spector \& Jex, 1998; Tjosvold, 1998), WIC presents a substantial obstacle for members of the organization to work together and meet 
organizational goals. Indeed, from this perspective, conflict can be viewed as a performance constraint, inhibiting people from cooperating with one another on the job (Spector \& Jex, 1998) or being able to complete a work task (e.g., Jehn, 1995). Thus, conflicts in the workplace may be especially difficult due to the fact that interactions with 'difficult' people at work may be unavoidable, particularly within work teams or groups (e.g., Jehn, 1995) and especially within the human service job sector (e.g., nursing, customer service; e.g., Barling et al., 2009; Farrell, 1999; Schat, Frone, \& Kelloway, 2006). Therefore, as a work performance constraint that is often unavoidable, WIC is a formidable source of work stress that can profoundly affect organizations.

Moreover, from an organizational perspective, WIC may be the single most important workplace stressor to address as it represents one of the largest reducible organizational costs (Dana, 1999; Keenan \& Newton, 1985). That is, organizations can substantially reduce costs by directly instituting conflict management and prevention policies among employees. For instance, WIC has been documented as directly predicting lower levels of work productivity and performance (Jehn, 1997; Pearson \& Porath, 2005; Tjosvold, 1991) as well as substantially contributing to increased work absenteeism (Tjosvold, 1991). Perhaps of most interest to organizations, WIC is a direct predictor of intentions to quit and employee turnover (Cortina et al., 2001; Frone, 2000; Spector \& Jex, 1998). Moreover, employee turnover is also indirectly affected by other WIC-related outcomes, including job dissatisfaction (Frone, 2000; Harvey, Blouin, \& Stout, 2006; Jehn, 1997), job burnout (Frone, 2000; Harvey et al., 2006; Leiter, 1991) and decreased motivation (Bergmann \& Volkema, 1989). Closely associated with employee 
turnover and additional costs to the organization, work disability is also affected by WIC. In a longitudinal examination of more than 15,000 employees, Appleberg et al., (1996) discovered frequent WIC, especially for women, was a significant risk factor for development of future work disability. Therefore, evidence suggests that WIC is producing high costs for organizations.

WIC can also spawn more severe social problems within the organization, which can further affect work productivity and organizational costs. In fact, conflict between individuals at work can directly lead to costly counter-productive work behavior (CWB; Bruk-Lee \& Spector, 2006), a form of retaliatory behavior that is contrary to organizational goals and can be directed either toward the instigator (e.g., supervisor, coworker) or toward the organization itself (Bennett \& Robinson, 2000). These behaviors produce numerous costs to the organization, such as work opportunity costs through lower work productivity and decreased employee morale. Of most concern, however, workplace violence and aggression are potential outcomes of WIC, particularly for those conflicts which are prolonged, unresolved, or handled poorly (Barling et al., 2009; Goldstein, 1994; Nolan, Shope, Citrome, \& Volavka, 2009; Pearson et al., 2001). Acts of physical workplace violence and aggression are particularly concerning due to their intense, physical nature and because they produce significant safety concerns for the employees (Schat et al., 2006; Herschcovis \& Barling, 2006). Additionally, workplace violence and aggression often produce psychological anxiety in those who witness the aggressive behavior, usually decreasing their own work productivity (Herschcovis \& 
Barling, 2006; Salin, 2003). Thus, severe social problems amongst employees are additional WIC outcomes of importance to organizations.

Employee or personal outcomes. As briefly outlined earlier, employees often rate WIC very negatively, indicating that WIC negatively affects them in profound ways. Indeed, interpersonal conflicts may be particularly distressing as many work relationships are central to the lives of employees, particularly in regards to their roles and social identities (e.g., Katz \& Kahn, 1978; Tajfel \& Turner, 1987). For instance, many employees indicate that coworkers provide essential social support (e.g., AbuAlRub, 2004; 2006; Beach et al., 1993) and these colleague relationships are a frequently cited reason to remain in their job after their first year of employment (Robinson, Murrells, \& Smith, 2005). Similarly, supportive relationships with supervisors have additional numerous positive benefits such as improved safety outcomes (e.g., Parker, Axtel, \& Turner, 2001), increased job satisfaction (e.g., Thomas \& Ganster, 1995), and lower levels of work stress (e.g., Kelloway, Sivanathan, Francis, \& Barling, 2005). Even relationships with those outside of the organization (e.g., customers, patients) can be important as a substantial reason to remain in the job, especially for nurses (e.g., Huey \& Hartley, 1988). Thus, WIC may be a particularly challenging source of personal stress due to the loss of a potentially positive relationship and the associated benefits (e.g., social support, satisfaction).

Moreover, WIC affects the health and well-being of employees. Depression and social withdrawal are of particular concern, as individuals exposed to frequent WIC often score higher on measures of depression and seek to withdraw from social interactions 
(Bruk-Lee \& Spector, 2006; Frone, 2000; Inoue \& Kawakami, 2010). WIC often engenders depressive thoughts that increase employee tendencies to avoid others in the social environment (Bergmann \& Volkema, 1989; 1994; Cosier, Dalton \& Taylor, 1991; de Dreu \& Beersma, 2005; Frone, 2000). Moreover, other psychiatric problems such as suicidal behavior and psychosis have also been identified as psychological outcomes of WIC. In fact, a prospective study investigating the long-term effects of frequent exposure to WIC by Romanov et al. (1996), found that WIC predicted increases in doctordiagnosed psychiatric morbidity one year later. As such, these studies suggest that WIC can have profound effects on the psychological well-being of employees.

Of additional concern, WIC has also been linked to marked decreases in physical health and social functioning. For example, in their meta-analysis of 18 separate samples, Spector and Jex (1998) found a significant positive association between health symptoms (e.g., flu, cold, headache) and greater frequency of experiencing WIC. More recently, de Raeve et al. (2009) found increased WIC frequency to significantly predict self-reported increases in fatigue, need for recovery, and decreased general physical health one year later in a sample of over 12,000 employees across 45 different companies in the Netherlands. Concerning social functioning, WIC leads to more negative interpersonal attitudes (Pruitt \& Rubin, 1986) and spawns further conflict and strife through confrontation, threats, physical force and aggression (Morrill \& Thomas, 1992; Sternberg \& Dobson, 1987). In fact, there is substantial evidence that conflicts can escalate according to a spiral, where each interaction between the parties becomes increasingly more negative or intense as each party reacts more negatively to each interaction. As the 
spiral continues, the conflict becomes more severe, eventually progressing to aggressive or violent behaviors (e.g., Andersson \& Pearson, 1999; Wall \& Callister, 1995). As such, WIC has the potential to markedly impact employee physical and social functioning in the workplace.

In summary, interpersonal conflicts at work are substantial stressors that have many negative effects on both organizations and employees. Organizational outcomes are disconcerting due to the sheer cost associated with increased turnover, lower productivity, and severe social problems. Employee outcomes of WIC related to psychological, physical and social functioning are no less disturbing. Taken together, these WIC outcomes highlight the importance of examining WIC and ways to reduce these negative effects. Next, I will discuss why it is important to examine WIC in the nursing context.

Workplace interpersonal conflict in nursing. Nurses are a population of workers where WIC outcomes can be particularly detrimental. The role of a nurse involves a high level of chronic work stress, which is associated with such negative outcomes as high turnover (e.g., Chang, Hancock, Johnson, Daly, \& Jackson, 2005), increases in medical errors and poor patient care (e.g., Elfering, Semmer, \& Grebner, 2006; Lambert et al., 2003), and development of various mental and physical health effects (e.g., burnout; Bakker, Kilmer, Siegrist, \& Schaufeli, 2000; Leiter, 2005). In fact, in a study of health records of 22,000 workers in over 130 occupations, the National Institute of Occupational Safety and Health (NIOSH) found health care personnel (e.g., RNs, nurse aides) showed higher than average incidence of health care utilization and 
medical visits (Smith, Colligan, \& Hurrell, 1977; Sulsky \& Smith, 2007). For this reason alone, investigation of nursing stress warrants attention. However, in addition to high levels of nurse work stress, the healthcare workplace itself presents multiple sources from which interpersonal conflict stressors can develop (e.g., nurse managers, physicians, coworkers, patients/families), leading to an overall high incidence and prevalence of WIC among nurses (e.g., Baltimore, 2006; Cheuk et al., 1997; Dewe, 1987; Dunn, 2003; Farrell, 1999; 2001; Leiper, 2005; McMahan, Hoffman, \& McGee, 1994; Wright, 2009).

Recent research has indicated nursing WIC is on the rise due to increased reliance upon teams to provide quality health care and high work demands associated with nursing (e.g., Chang et al., 2005). For instance, conflicts between nurses themselves are rapidly escalating in terms of both frequency and severity, due to many factors such as frustrations with work load, shortages of nurses and feeling unfairly treated by fellow nurses (e.g., Baltimore, 2006; Dunn, 2003; Farrell, 2001; Thobaben, 2007; Wright, 2009). Conflicts with nurse managers, physicians, and patients or their families are also of particular concern, as these often have serious potential consequences for quality of work and patient care (Baggs \& Ryan, 1990; McMahan et al., 1994; Tabak \& Koprak, 2007). Some studies have estimated that nurse coworkers are the most prevalent source of conflict (e.g., Dunn, 2003; Farrell, 1999; 2001) whereas others have suggested patient and family conflicts occur more often than reported, especially verbal maltreatments (e.g., Adip, Al-Shatti, Kamal, El-Gerges, \& Al-Raquem, 2002; Billeter-Koponen \& Freden, 2005). However, the literature agrees that nursing WIC is a significant problem adversely affecting nurses and is an area that needs further empirical and theoretical 
attention (Almost, 2006; Dunn, 2003). As such, this dissertation focused on coping with nursing WIC to identify mechanisms by which nurses can deal with these conflicts effectively.

In summary, workplace interpersonal conflict may best be defined as a multidimensional construct comprised of cognitions (e.g., disagreement), behaviors (e.g., interference), and/or negative emotion (e.g., frustration) within situations of interdependence between persons. WIC is associated with many negative organizational and employee outcomes, making it a stressor of particular concern, especially for nurses. As WIC presents a formidable stressor that produces a wide variety of negative outcomes, employees are faced with the difficult decision of how to deal or cope with these stressful conflicts. As such, in the next chapter I will discuss workplace stress and coping theory, which provided the theoretical framework for examining nursing WIC. 


\section{Chapter 3: The Transactional Model of Stress and Coping}

Stress, as a phenomenon often associated with negative outcomes, has garnered much attention within the organizational literature. In fact, a recent estimate has placed stress as the dominate subject of research inquiry across all journals in occupational health psychology for the past several decades (Macik-Frey, Quick, \& Nelson, 2007). Moreover, in attempts to investigate stress, several models and theories have been developed regarding causes, outcomes, and the resulting strain of prolonged or chronic stress, especially within the workplace context (e.g., Bakker, Demerouti, De Boer, \& Schaufeli, 2003; Karasek, 1979; Siegrist, 1996). Although many stress models exist, perhaps none have been more influential than the transactional model of stress and coping as put forth by Lazarus and Folkman (1984). In this chapter, I detail the transactional model of stress and coping, which forms the theoretical foundation for this dissertation. Before I elaborate this model, I want to direct the reader to Appendix A for a glossary of acronyms used in this dissertation since many of these arise in this chapter. Below, I briefly discuss prominent work stress models and explain why the transactional model provides the optimal perspective when examining nurse coping with WIC.

\section{Overview of Prominent Work Stress Models in the Literature}

As work stress began to be increasingly recognized as important in the workplace, the Job Demands-Control (JDC) model was developed depicting job strain as the result of an interaction between job demands and job control (i.e., job decision latitude; Karasek, 1979). More specifically, the most adverse strain outcomes were theorized to be produced under conditions of high job demands and low perceived control (Karasek, 1979; Karasek 
\& Theorell, 1990). Using this model, empirical investigations have focused on two main hypotheses in predicting job strain: a main effect of job demands on strain and a buffering effect of control on this relationship (van der Doef \& Maes, 1999). Throughout the literature, there has been mixed support for this model. For instance, in a review of 51 studies, van der Doef and Maes (1999) found substantial support for the main effect of high job demands on such job strain indicators as cardiovascular problems and negative pregnancy outcomes, but only limited or inconsistent support for gastrointestinal disease, psychosomatic complaints, and musculoskeletal symptoms. Moreover, van der Doef and Maes (1999) only found partial support for the buffering hypothesis and no support for either hypothesis in the longitudinal designs they examined.

In a more recent review of this literature, de Lange, Taris, Kompier, Houtman, and Bongers (2003) reviewed articles examining the JDC model that were considered high-quality studies. Each of these studies were selected based upon five methodological criteria: 1) panel design (enabling examination of standard, reversed, and reciprocal causal relationships), 2) theoretical justification for time lags used, 3) high quality measures, 4) high quality analytic techniques (i.e., multilevel regression, structural equation modeling), and 5) missing data analysis. Despite using these "high quality" studies, de Lange et al. (2003) reported similar results as earlier reviews with only modest support for the JDC Model. Therefore, although the JDC model has been incredibly influential in work stress theory and empirical investigation, the literature support is modest at best, suggesting that this model gives a partial, but likely not complete, depiction of work stress and strain. 
In an attempt to examine work stress and strain using variables other than job demands and control, Siegrist (1996) proposed the Effort-Reward Imbalance (ERI) model. Accordingly, job stress and strain is modeled as a function of an imbalance between the efforts an employee exerts at work and the reward or compensation the individual receives for these work-related efforts. The ERI model contains three main propositions including 1) the extrinsic ERI hypothesis: high efforts with low rewards produce work strain (i.e., poor health), 2) the intrinsic overcommitment hypothesis: high levels of overcommitment can increase poor health, and 3) the interaction hypothesis: employees with extrinsic ERI and high overcommitment are even more susceptible to work strain. Empirical investigations have demonstrated that the ERI model predicts work strain outcomes including physical health, behaviors, and psychological well-being (van Vegchel, de Jonge, Bosma \& Schaufeli, 2005). Throughout the literature, however, there has been mixed support for this model. In fact, in their review of 45 prior studies examining the ERI model, van Vegchel et al. (2005) found considerable support for the extrinsic hypothesis, but very little or inconsistent support for the overcommitment and interaction hypotheses.

Moreover, the ERI model has suffered from other difficulties. First, the literature has often failed to produce any findings supporting a stressful reaction to an imbalance of low efforts and high rewards, suggesting that an imbalance between effort and reward may not be the principle mechanism of work strain (van Vegchel et al., 2005). In connection with this, the inconsistent support (at best) for the overcommitment hypothesis suggests that this model may not be appropriately depicting the work stress- 
strain relationship. Second, the ERI model inherently reduces the work stress-strain relationship to only a few variables, potentially oversimplifying the work stress process. Although limited efforts have been made to incorporate aspects of other models (e.g., Rydstedt, Devereux, \& Sverke, 2007), the ERI model generally fails to acknowledge the important role that other variables (e.g., job autonomy, resources) may have in the stressstrain relationship. As such, despite the literature support for the extrinsic ERI hypothesis, the ERI model also provides only a partial explanation for work stress and strain.

In efforts to build upon the static character of both the JDC and the ERI models, Bakker et al. (2003) proposed the Job Demands-Resources (JDR) model. Whereas the JDC and ERI models focus on specific factors such as job control and rewards, the JDR takes a broader approach, encompassing a wide-array of variables representing resources which can be used to meet job demands. According to this model, job demands and job resources operate in a dual process where job demands have a negative main effect on strain and job resources have a positive main effect on work motivation. Moreover, job resources are depicted as buffering the negative effects of job demands on strain. Indeed, consistent with Conservation of Resources Theory (COR, Hobfoll, 1989; Hobfoll \& Lilly, 1993), the JDR proposes that any resource available to the employee, especially those already gained, are essential components in the work stress-strain relationship. In their review of several studies across diverse occupations, Bakker and Demerouti (2007) found support for the JDR model's dual process predictions and partial support for the 
JDR model's buffering hypothesis of resources on job demands. Indeed, the JDR model, for many researchers, represents the most advanced work stress model available.

Although the JDR is the most recent model proposed within the literature, it is not without its limitations. Perhaps most importantly, the JDR struggles to explain how individual differences in responses to work stressors can occur under objectively similar job demands and resources. For instance, in a recent study of HIV/AIDS caregiving volunteers, Cox, Pakenham and Cole (2010) found only partial support for the JDR model. Specifically, in support of the model, they found that job demands were predictive of volunteer burnout (health outcome) and job resources were related to satisfaction (motivational outcome). However, these effects were not independent of one another, as job demands were related to both health and motivational outcomes and job resources were also related to both, which does not support the JDR model. As such, these results suggest that individuals may approach each job demand and resource with different psychological attitudes. For instance, two employees who have the same resources available to them at work (e.g., social, managerial) may respond very differently when faced with a challenging task deadline. Whereas one may thrive under the pressure of the deadline and improve in job performance, the other may become distressed and develop health problems. Hence, external characteristics such as job demands cannot be assumed to always have negative effects and, similarly, job resources to have positive effects. This further suggests that a subjective process may operate beyond the objective conditions of job demands and resources. 
Moreover, the JDR focuses almost exclusively on characteristics of the external context, paying little attention to internal processes such as cognition or emotion. Such variables can profoundly affect responses to stressful situations (e.g., Lazarus, 2006). For instance, negative appraisals of job demands may exacerbate the detrimental effects of these job demands and negate any positive benefits that could come from available job resources. Similarly, positive appraisals of job demands may mitigate the harmful effects of job demands and enhance the benefits of job resources. This is supported by a few recent studies examining the JDR. For example, van den Broeck, de Cuyper, de Witte, and Vansteenkiste (2010) reported that job demands appraised as either job hindrances or job challenges were differentially supportive of the JDR model. Specifically, those job demands considered a hindrance (e.g., work is emotionally demanding) supported the JDR model, whereas job demands considered a challenge (e.g., work is fast-paced) did not provide support. These findings suggest that the JDR model's explanatory power may be couched within subjective cognitive evaluations of job demands. That is, an individual must cognitively consider a job demand to be taxing or stressful in order for it to have a detrimental effect on the individual. However, the JDR model does not include subjective evaluations of job demands/resources and lacks a cognitive or affective component in the stress process. Therefore, although the JDR provides a work stress model that improves upon the JDC and ERI models, the JDR model struggles to explain individual differences in stress outcomes. 


\section{The Transactional Model of Stress and Coping}

In their seminal work on stress and coping, Lazarus and Folkman (1984) introduced cognitive appraisal as the subjective process explaining individual differences in stress and strain. Taking a different approach from other models, Lazarus and Folkman (1984) argue that the individual's cognitive appraisal or subjective evaluation of stressor characteristics occupies the critical role of determining perceived stress levels and, ultimately, the individual's behavioral response to the stressor (see Figure 3). As an individual is exposed to a particular encounter, the individual undergoes cognitive appraisal to determine if it is stressful or not and, if it is, how best to handle or cope with the event. Hence, any event could potentially be considered a stressor, depending upon the individual's subjective appraisal of the situation. The transactional model has received a great deal of empirical support in the literature (e.g., Folkman \& Lazarus, 1985; DeLongis \& Holtzman, 2005; Zakowski et al., 2001), including within the workplace context (e.g., Lazarus, 1991; Peeters, Buunk, \& Schaufeli, 1995; Spell \& Arnold, 2007). Moreover, other theoretical perspectives developed after the transactional model (e.g., COR Theory, Hobfoll, 1989), have been criticized as merely restating the transactional model in different terms (Lazarus, 2001), suggesting that the transactional model provides a comprehensive model and depicts the chief underlying mechanism for work stress and strain.

First, the primary advantage of the transactional model is its ability to examine stress and coping as a subjective process, explaining both between- and within-person differences. Indeed, whereas the JDC, ERI and JDR models implicitly assume that job 
demands will affect the individual in similar ways throughout time, the subjective process of the transactional model captures both between-persons differences (similar to these other models) and change over time as the individual adapts to the stressor and environment. For example, although the demands of a short deadline in which to accomplish a specific work task may be stressful at one point for an individual, a similar short deadline may not always be stressful, even for that same individual. Individual variation such as this has frequently been a challenge to explain using these other work stress models. Therefore, using the transactional model, one can explain these individual differences in the stress-strain process beyond external job demands by accounting for the complex internal cognitive appraisal process that differ between- and within-persons over time. Moreover, whereas the JDC, ERI and JDR models assign the individual a more passive role in responding to stressors, the transactional model depicts the individual as cognitively processing information and then interacting with the environment to attain beneficial outcomes. As such, the transactional model offers an optimal model by depicting work stress as a subjective process that both differs between-persons as well as within-persons over time.

Second, the transactional model contains a comprehensive framework of responses (i.e., coping) to stressors, constituting another major advantage over these other work stress models. Whereas the JDC, ERI, and JDR models propose mechanisms that explain work stress development, they stop short of expounding upon individual responses to stress. These models do not contain explanations or predictions of how an individual will cope with work stress once it has been produced. In other words, the end 
point for these models is work strain (e.g., burnout, turnover). The transactional model, on the other hand, provides a model explaining the development of work stress, a comprehensive classification of coping behaviors the individual can engage in, and specific predictions regarding the effectiveness of these coping behaviors (Lazarus \& Folkman, 1984; Zakowski et al., 2001). Therefore, the transactional model provides a more comprehensive approach to examining the stress process than the other models. As coping is the focus of this dissertation, I will return to this topic again in more detail later in this chapter.

Hence, by taking a subjective process-oriented perspective and considering the entire stress process including coping, the transactional model improves upon most of the primary limitations of these other work stress models. In fact, these strengths make the transactional model a logical choice when examining stressors which are particularly prone to subjective differences and elicit a coping response, such as WIC. As I used this model in my dissertation, I discuss the specific tenets of cognitive appraisal and coping below.

\section{Cognitive Appraisal}

According to the transactional model, a stressor is any potentially threatening or demanding event or encounter appraised as stressful that requires an adaptive response on the part of the individual. Therefore, the individual undergoes cognitive appraisal of potentially stressful events to determine whether the event is threatening or demanding before responding to the stressor. Specifically, cognitive appraisal includes three distinct

processes that may not necessarily occur in this order: primary appraisal, secondary 
appraisal, and reappraisal (see Figure 3; Folkman \& Lazarus, 1985; Lazarus \& Folkman, 1984). I discuss each of these below.

Primary appraisal. Generally considered as the first step in the appraisal process, primary appraisal occurs as an individual assesses whether an event or encounter is directly applicable to the individual (Lazarus \& Folkman, 1984). This embodies the three potential outcomes for the individual by interpreting the event as 1) irrelevant (not pertinent), 2) positive or benign (not threatening), or 3) stressful (harmful, threatening or challenging). In the first case, if an event is considered to be irrelevant, the individual will have no need to respond to the potential stressor, as it does not directly apply to the individual. Likewise, if the encounter is appraised as positive or benign, a coping response is not warranted, as it does not pose a threat or challenge to the individual's well-being. In the case of a stressful event, however, the individual decides the event has already caused personal harm, the event represents a threat of future personal harm, or the event is considered a challenge (see Figure 3). For example, a nurse may witness another nurse abusing internet privileges by looking up vacation pictures while in the operating room when they should be working. As such, the nurse may decide this event is personally relevant and represents a threat of future harm (likely need to perform additional work to make up for coworker negligence), thereby resulting in a primary stress appraisal for this WIC.

Lazarus and Folkman (1984) further differentiate between the specific primary stress appraisals of threat and challenge. Although both of these appraisals call for the mobilization of coping efforts, challenge appraisals focus on the potential benefits or 
growth inherent in the stressful encounter and are characterized by pleasurable emotions such as exhilaration and excitement. On the other hand, threat appraisals center on the potential harm to the individual with negative emotions such as anger or fear. Threat and challenge appraisals, though clearly different in terms of cognitions (i.e., potential harm versus gain) and affect (i.e., positive versus negative emotions), are not mutually exclusive, particularly as a stressful encounter unfolds over time. Continuing with the prior example, the nurse may feel challenged to perform the additional job tasks of the negligent coworker, which would be accompanied by feelings of excitement and thoughts about the potential growth from this opportunity. More likely, however, the same nurse may make a threat appraisal of the same conflict as the unprofessional and distracting behavior of the coworker is creating more work for the nurse, which brings about negative feelings such as anger or anxiety and thoughts about the potential harm that could come from this conflict (e.g., losing job because of coworker). As such, the difference between threat and challenge appraisals partly explains individual differences observed in responding to objectively similar stressors. Upon making a harm, threat or challenge appraisal, the individual next engages in a complex evaluative process called secondary appraisal in order to cope with the stressful event.

Secondary appraisal. As delineated by Lazarus and Folkman (1984), primary appraisals interact in a complex process with secondary appraisals to determine the level of stress an individual experiences and the strength of the emotional reaction. Secondary appraisal involves the identification of what might and can be done in the stressful situation. As the outcome of any stressful encounter relies upon what, if anything, can be 
done and what is at stake, secondary appraisal represents a crucial aspect of the appraisal process. According to the transactional model, two particular factors affect this appraisal process, including commitments and beliefs (Lazarus \& Folkman, 1984). Specifically, an assessment of the level of commitment or the stakes one has in the stressful encounter (i.e., importance of the stressor and associated outcomes) as well as the individual's belief in the amount of power or control over the stressful situation and outcomes are intimately tied to what might and can be done in the situation.

First, the level of commitment one has to the stressful situation influences the secondary appraisal of the encounter (Lazarus \& Folkman, 1984). When one feels that the stressor or the outcome of the stressful encounter is of great importance or holds particular meaning, the individual has a high level of commitment. If the individual is highly committed, the individual may consider more ways to respond to the stressful situation or be more motivated to find ways to resolve the stressor. The inverse should also be true, so that when the individual is not highly committed, few additional ways to respond to the stressful encounter may be sought or motivation to completely resolve the stressor may be low. Using my example of the nurse observing a colleague abusing internet privileges, the level of commitment or importance of the nurse behavior may affect the appraisal of the conflict. Specifically, if the nurse considers professional nurse conduct an important aspect of nursing (e.g., image, job description), the nurse will likely consider more options or make more efforts to resolve the conflict effectively.

Second, an individual's perceived control in the stressful encounter is a vital contextual factor influencing secondary appraisal. The individual's general perception of 
control concerns the extent to which the individual believes he/she can alter events and outcomes of importance in the situation or change the environment to make it more rewarding or less threatening (Ganster, 1989; Lazarus \& Folkman, 1984). Even though there may be a great deal of variability across individuals in how control is defined, the transactional model is built upon the premise that perceived control should be subjectively defined (Lazarus \& Folkman, 1984). Subjective definitions of control permit within-person comparison of perceived control over time, as perceptions of control across different situations (i.e., situational control appraisals) for the same person can be compared to one another. Therefore, any fluctuations in perceived control can be attributed to actual changes in control rather than differences between people in how control is defined. Indeed, an individual's perception of control across stressful situations is much more likely to fluctuate than an individual's definition of control (Lazarus \& Folkman, 1984), making subjective definitions of control an essential component of the transactional model.

Perceptions of control also influence secondary appraisal through limiting or expanding the perceived ways one might respond or cope with the stressor. Under situations of high appraised control, for instance, one would likely believe more coping options are available, whereas under low control appraisals, fewer available coping options would be perceived. Continuing with the earlier example, the nurse may perceive a low amount of control in the conflict, perhaps due to multiple nurses engaging in the abuse of internet privileges. As such, the nurse may feel fewer options are available to influence several colleagues in order to effectively resolve the conflict. Or, the nurse may 
perceive a high amount of control in the conflict, perhaps due to other colleagues similarly condemning this behavior, leading the nurse to believe more coping responses are available to resolve the conflict. As appraisals of perceived control form the basis of the goodness of fit hypothesis and the focus of this dissertation, I will return again to appraisals of perceived control in my discussion of the goodness of fit hypothesis in Chapter 4.

Reappraisal. As a modification of an original primary or secondary appraisal, reappraisal refers to a reassessment of the stressor based upon new information received from the environment concerning the stressor or the outcomes of coping efforts. Under reappraisal, the relationship with the stressor is reevaluated and further consideration is made regarding continuing or altering the current coping strategy (see Figure 3; Lazarus \& Folkman, 1984). In essence, reappraisal is a continuation of both primary and secondary appraisal processes. As such, reappraisal represents the evaluative process by which individuals may change their coping strategies in dealing with a stressor over time. Thus, the transactional model takes into account individual variation following the initial appraisal and coping process in two ways. First, the reappraisal process describes change in coping efforts over time between-persons, as these changes are contingent upon individual subjective evaluations of the stressors and coping outcomes (DeLongis \& Holtzman, 2005; Peeters et al., 1995). Second, the reappraisal process explains the within-persons variation often evident when examining coping with stressors over time (e.g., Folkman \& Lazarus, 1985; Drumheller, Eicke, \& Scherer, 1991). Thus, coping efforts that change over time simply reflect the results of reappraisals of chronic or 
recurring stressors. In my example, the nurse may initially appraise the conflict as one of low perceived control based upon multiple nurses participating in the unprofessional behavior. However, upon learning that management prohibits this behavior, the nurse may then reappraise the conflict as one where he/she has more control over ways to cope with or resolve the conflict.

In summary, cognitive appraisal is the process whereby individuals evaluate potential stressors and these appraisals then influence the response to the stressful encounter. Specifically, primary appraisal is the process whereby the individual determines that an environmental stimulus is a stressor as it has already caused harm, threatens future harm, or presents a challenge. These primary stress appraisals lead the individual to mobilize coping responses to deal with the stressor and engage in another cognitive evaluation, secondary appraisal. Secondary appraisal involves determining what the individual might and can do to respond to the stressor, which is affected by level of commitment to and perceived control over the stressor and outcome. Finally, based upon additional information regarding the stressor or coping efforts, reappraisal is the process whereby the earlier appraisal is modified. Thus, the individual makes coping efforts based upon cognitive appraisals of the stressor. I proceed to discuss coping below, paying particular attention to the transactional model's classification of coping efforts (i.e., problem-focused, emotion-focused), coping effectiveness, and coping in the nursing context. 


\section{Coping}

As a term that is commonly used both in science and in the common vernacular, coping has come to represent a wide-array of meanings and behaviors over time (Lazarus, Averill, \& Opton, 1974; Parker \& Endler, 1996). For instance, coping originally began as a study of how individuals responded to extreme situations or major life events (e.g., Hamburg, 1974; Henderson \& Bostock, 1975), but has since evolved to include more common situations such as daily stressors and hassles (e.g., Kohn, 1996; Porter \& Stone, 1996; Stone \& Neale, 1984; Tennen \& Affleck, 1996). Moreover, measures are also diverse in both derivation and focus as most are either derived by empirical results (e.g., Ways of Coping Questionnaire, Folkman \& Lazarus, 1988) or from theory (e.g., COPE, Carver, Sheier, \& Weintraub, 1989) and focus on either situation-specific coping (e.g., measure of daily coping; Stone \& Neale, 1984) or dispositional coping (e.g., Coping Inventory for Stressful Situations, Endler \& Parker, 1990). Similarly, many different classifications and typologies of coping behavior have been developed within the literature (e.g., Endler \& Parker, 1990; Latack, 1986; Lazarus \& Folkman, 1984). In this section I begin by defining coping, briefly discuss prominent coping classifications in the literature, and then detail the transactional model's differentiation between problemfocused and emotion-focused coping.

Coping defined. Most researchers have come to consensus that coping represents an individual's response to a stressor or stressful situation (e.g., Parker \& Endler, 1996). Moreover, most researchers also agree that coping is a conscious process whereby the individual negotiates both personal (e.g., morals, beliefs) and environmental (e.g., 
situation) demands to deal with the stressor (Haan, 1992; Latack, 1986). Indeed, within a long line of theoretical and empirical work, Lazarus and colleagues have offered a definition of coping as "constantly changing cognitive and behavioral efforts to manage specific external and/or internal demands that are appraised as taxing or exceeding the resources of the person" (Lazarus \& Folkman, 1984, p. 141). This definition is incredibly versatile, as virtually any type of behavior can be considered coping if enacted to manage demands of the stressor from the perspective of the individual. As this definition offered by Lazarus and Folkman (1984) in the transactional model is widely considered the most comprehensive and accurate definition of coping (Parker \& Endler, 1996; Zakowski et al., 2001; Zeidner \& Saklofske, 1996), this was used throughout this dissertation.

Before leaving this definition, however, it should be noted that the literature does not uniformly agree that coping is constantly changing (see Cohen, 1987; Folkman \& Lazarus, 1981; Shinn \& Krantz, 1981). Indeed, another line of research has taken a traitbased approach, modeling coping as stable, consistent patterns of behavior resistant to change across time and situations and manifested as a person's preference or natural proclivity to cope with stressors in certain ways (Carver et al., 1989; Compas et al., 1988; Pearlin \& Schooler, 1978; Vaillant, 1977). Opposing the view of coping as constantly changing (i.e., state perspective), this trait perspective maintains that coping is a stable set of behavioral patterns and that any variation is due to individual differences. As both perspectives have garnered considerable support during the past several decades (e.g., Compas et al., 1988; Costa, Somerfield \& McCrae, 1996; DeLongis \& Holtzman, 2005; Lu, 1996; O’Brien \& DeLongis, 1996; Schwartz, Neale, Marco, Shiffman, \& Stone, 
1999; Todd, Tennen, Carney, Armeli, \& Affleck 2004; Vailliant, 1977), it seems there is evidence of influence of both personality and situation on coping behavior.

Consistent with the transactional model, considering coping as a process wherein behaviors change continually according to situational characteristics and cognitive appraisals has several advantages (Folkman \& Lazarus, 1985). For example, a primary advantage of this coping state perspective is the ability to account for differences in coping above and beyond what average levels of coping would indicate. Indeed, global retrospective reports of coping (i.e., trait coping, average levels) are often not accurate in predicting what individuals actually do to cope with stressors (Stone et al., 1998; Todd et al., 2004) and often fail to predict the ebb and flow of coping patterns over time (Tennen \& Affleck, 1996). Moreover, although patterns or consistencies in coping may be exhibited within individuals across time (e.g., Lu, 1996; Schwartz et al., 1999), variations within the sequences or combinations of coping strategies may best be explained as a function of specific situational characteristics, such as cognitive appraisal (Lazarus \& Folkman, 1984; Tennen \& Affleck, 1996). As such, by using the transactional model in this dissertation, I was able to examine individual differences in coping between-persons and differences over time within-persons.

Coping classifications. As I highlighted earlier, one of the greatest contributions the transactional model has to offer is a comprehensive framework of coping behaviors. Indeed, the transactional model differentiates coping behaviors based upon the aim of the coping effort as either problem-focused coping (i.e., change or modify the stressor itself) or emotion-focused coping (i.e., regulate emotional response to the stressor). As such, the 
coping classification of problem-focused and emotion-focused represents a wide assortment of cognitive processes and behaviors that could be considered coping (Lazarus \& Folkman, 1984). A substantial literature has examined these coping efforts, particularly in regards to conditions under which they occur (e.g., Bolger, 1990; Ptacek, Smith \& Dodge, 2000), their interrelationship (e.g., Baum, Fleming, \& Singer, 1983; Tennen et al., 2000), and how effective they are in mitigating the effects of the stressor (e.g., Aldwin \& Revenson, 1987; Zeidner \& Saklofske, 1996).

Although the transactional model's differentiation of coping behaviors into problem-focused or emotion-focused coping is often considered the most prominent classification in the literature (e.g., Zakowski et al., 2001), there have been other approaches proposed in the literature. For example, agreeing that appraisal of situations influences coping in the workplace, Latack (1986) proposed that coping with work stress should be classified as either control or escape. Accordingly, control coping consists of both actions and cognitive reappraisals that are proactively aimed at exerting control over the stressful situation and escape coping is defined as consisting of actions and cognitive reappraisals geared towards escaping or avoiding the stressor altogether. In another coping framework, Endler and Parker (1990) suggest that coping be classified under one of three types: task-oriented, emotion-oriented, or avoidance-oriented. This approach, they argue, captures specific dimensions of coping, especially in relation to coping style (trait-based approach).

Two additional, more recent, alternative coping frameworks are also important to note. First, in a review of the coping literature devoted to child and adolescent coping, 
Skinner, Edge, Altman and Sherwood (2003) argue that the higher order categories of action types (e.g., proximity seeking, mastery, accommodation) should be used rather than dimensions or functions. This is because, at least in the context of child development, any type of coping is likely to be multidimensional and potentially serve many functions. Second, developed by Neal and Hammer (2007), another classification depicts coping as emotional, cognitive and behavioral types. This approach has proven particularly applicable when examining coping with stressors within the work-family context (Neal \& Hammer, 2009). Although all of these classifications present alternatives, the classification of problem-focused and emotion-focused coping as advocated by the transactional model, provides a more encompassing and validated approach to coping for a few reasons.

First and foremost, the problem-focused and emotion-focused coping distinction offers the most comprehensive coping classification currently available. Indeed, problemfocused and emotion-focused coping can be used to classify any coping behavior that is used to manage the stressful encounter, regardless of any situational characteristic. The problem/emotion-focused coping distinction is not limited to any one context, representing coping that may occur across multiple contexts (e.g., work, home). Moreover, as opposed to other classifications (e.g., escape), problem/emotion-focused coping does not initially assume any coping effort to uniformly produce undesirable outcomes. As such, any coping behavior can be considered as potentially effective, rather than assuming one type of coping to be universally ineffective. Finally, according to the coping literature, the problem/emotion-focused coping distinction continues to have the 
most support of all coping classifications (e.g., Zeidner \& Saklofske, 1996; Zakowski et al., 2001), strongly suggesting that this classification is the best available. As such, I used the problem-focused/emotion-focused coping classification and briefly outline this literature below.

Problem-focused and emotion-focused coping. According to Lazarus and Folkman (1984), problem-focused coping strategies are coping efforts directed at the problem or stressor itself, usually in attempts to manage or change the stressor. Problemfocused coping represents the individual's efforts to alter, manage, or eliminate the problem or stressor. Moreover, these can include efforts directed at defining the problem or generating alternative solutions. Problem-focused coping embodies more than just problem solving, since problem solving is primarily directed towards the environment and problem-focused coping can be inwardly directed. For example, cognitive reappraisal of a stressor to change motivations or cognitions such as shifting level of aspiration or finding alternative channels of gratification is inwardly directed problem-focused coping (Lazarus \& Folkman, 1984). Although it may appear that fewer problem-focused coping strategies are available compared to emotion-focused coping strategies across the broad domain of potential stressors, many problem-focused coping strategies become apparent when examining specific situations. For example, the nurse dealing with the WIC involving the nurse abusing internet privileges may be able to use one or multiple problem-focused coping techniques such as directly approaching the nurse colleague, making a cognitive reappraisal to reduce the importance of the situation, or speaking with a supervisor regarding the situation. 
Emotion-focused coping, on the other hand, is aimed at regulating the emotional response and distress that the stressor elicits within the individual (Lazarus \& Folkman, 1984). Indeed, emotion-focused coping efforts are directed at helping the individual to maintain hope and optimism despite circumstances. As such, these coping efforts represent a wide range of coping behaviors that do not change the objective situation, such as cognitive reappraisals that seek to find the positive in the situation (e.g., positive comparisons), distancing, selective attention, or minimization (Lazarus \& Folkman, 1984). Moreover, emotion-focused coping includes avoidance behaviors (i.e., avoiding the stressor) as the emotional response to the stressor is regulated, but the stressor itself is not modified or managed. Using the same example of the conflict between the nurse and nurse colleague inappropriately using the internet, the nurse may engage in one or multiple forms of emotion-focused coping to cope with the conflict such as positively reappraising the conflict to focus on positive aspects of it, seek emotional social support from family or friends, or completely avoid future interactions with the other colleague. Thereby, emotion-focused coping represents a diverse range of strategies to reduce negative responses, promote positive reactions, and limit exposure to the stressor.

In addition to the sound theoretical support in the literature (Zeidner \& Saklofske, 1996), the distinction between problem-focused and emotion-focused coping has been examined and supported across numerous empirical studies. For instance, Folkman and Lazarus (1980) originally developed this distinction based upon both empirical data and theoretical reasoning when creating the Ways of Coping Checklist (WCC). Good internal consistencies were reported for both problem-focused and emotion-focused coping, 
suggesting the distinction was appropriate. In another study, Aldwin, Folkman, Shaefer, Coyne, and Lazarus (1980) found further support as their factor analysis of the WCC produced seven interpretable factors: one representing problem-focused and six representing emotion-focused coping.

Using the Ways of Coping Questionnaire (WCQ), a revised scaling of the WCC, Folkman and Lazarus (1985) reported evidence for eight subscales (six emotion-focused, one problem-focused, and one mixture of both) based upon a factor analysis of 324 completed questionnaires of undergraduate students pooled across three observation points. This analysis produced acceptable to good internal consistency reliabilities for all eight subscales, ranging from .56 to .85 . In another study using the WCQ, 750 observations from 150 adults were factor analyzed and examined for internal consistency (Folkman et al., 1986). These analyses produced similar findings to those reported by Folkman and Lazarus (1985) resulting in eight coping subscales with moderate reliability, ranging from .61 to .79 . Therefore, the distinction between problem-focused and emotion-focused coping is supported by both theoretical and empirical evidences in the literature.

Furthermore, notable differences in outcomes between problem-focused and emotion-focused coping provide further support. For instance, most studies generally report negative outcomes (e.g., distress, physical and mental health deficits) associated with greater use of emotion-focused or avoidance coping (e.g., Aldwin \& Revenson, 1987; Bianchi, 2004; Lambert et al., 2004). On the other hand, positive outcomes (e.g., decreased distress, high job satisfaction) are typically related to greater use of problem- 
focused coping (e.g., Compas et al., 1988; Parasuramon \& Hansen, 1987; Xianyu \& Lambert, 2006). It should be noted, however, that most of these studies have not examined specific stressors and appraisals in relation to specific types of coping as the transactional model posits. Indeed, Tennen et al. (2000) suggests these general results may be confounded with measurement, such that emotion-focused coping is associated with negative outcomes because it is used under more negative conditions that are not amenable to change by problem-focused coping. Moreover, several studies have identified conditions under which the general association of negative outcomes with emotion-focused coping and positive outcomes with problem-focused coping may not be true (e.g., Baker \& Berenbaum, 2007; Carver et al., 1993). For example, contrary to traditional expectations, Carver et al. (1993) reported emotion-focused coping such as acceptance and using humor were prospectively predictive of less distress in a sample of women with early stage breast cancer, but problem-focused coping strategies were not.

Other studies have further probed the relationship between problem-focused and emotion-focused coping. For instance, evidence suggests that problem-focused and emotion-focused coping are not mutually exclusive. This may be especially true for chronic or recurring stressors where problem-focused and emotion-focused coping may be used concurrently (Baum et al., 1983; Folkman \& Lazarus, 1985) or consecutively (Holtzman, Newth, \& DeLongis, 2004; Lazarus \& Folkman, 1984; Tennen et al., 2000). For example, in their study of undergraduate students taking a college exam during three stages over two weeks, Drumheller et al. (1991) found that students engaged in a variety of coping strategies. They reported that although the frequency of coping changed 
between stages, the use of both problem-focused and emotion-focused coping was reported at each stage, suggesting that students were using both types of coping simultaneously to deal with the stressor.

In another example, Tennen et al. (2000) examined how individuals suffering chronic pain from rheumatoid arthritis coped with daily pain. Using daily diary methodology, which involves respondents providing daily reports of their pain and coping over several weeks, Tennen et al. (2000) reported that on most days, both problem-focused and emotion-focused coping were used together rather than separately. They also found problem-focused coping often preceded the use of emotion-focused coping, suggesting that emotion-focused coping may follow problem-focused coping that is insufficient or ineffectual in dealing with the pain. Thus, using the earlier nurse example, the nurse may simultaneously use problem-focused and emotion-focused coping by directly confronting the offending nurse coworker to resolve the conflict while also seeking emotional social support from friends and family. Or, the nurse may use problem-focused and emotion-focused coping consecutively by seeking emotional support from family or friends following the failure (or limited success) of directly confronting the nurse colleague. In summary, these empirical outcome differences between problem-focused and emotion-focused coping provide additional support for this coping classification.

Finally, it should also be noted that certain individual differences also affect the use of problem-focused and emotion-focused coping strategies. As this dissertation is focusing on nurses, which is primarily composed of women, gender is particularly 
pertinent. Indeed, gender differences in coping have emerged as women consistently report employing more emotion-focused coping strategies than men and men report using more problem-focused coping strategies than women overall (e.g., Gonzalez-Morales, Peiro, Rodriguez, \& Greenglass, 2006; Krajewski \& Goffin, 2005; Ptacek et al., 1994). For example, in a study examining differences in coping by gender, Ptacek et al., (1994) had 59 women and 55 men undergraduate students give objectively similar lectures in front of research assistants who the participants were led to believe were rating them on their performance. Participants then provided self-reports of how they coped with the experience. Ptacek et al. (1994) reported that women were significantly more likely to rely upon social support and men consistently used problem-focused coping more than women in dealing with the stressor. Thus, consistent with these results, in a population comprised of a majority of women (such as nurses), the reported use of emotion-focused coping may be inflated.

As these studies suggest, coping involves a complex process whereby individuals attempt to address stressors using different types of coping efforts, making the effectiveness of such strategies in mitigating the harmful effects of the stressor a question of increasing importance. As such, I briefly discuss coping effectiveness below.

Coping effectiveness. Within the coping literature, there have been many efforts made to identify effective coping behaviors. Usually referred to as adaptive coping, effective coping refers to coping strategies that produce beneficial outcomes for the individual, such as increased self-esteem or decreased emotional distress (e.g., Lazarus, 1993; Zeidner \& Saklofske, 1996). Such terminology also suggests that individuals vary 
their coping responses to meet or match environmental characteristics or demands (Lazarus, 1993; Lazarus \& Folkman, 1984). Thus, effective coping is widely considered to be coping efforts that yield beneficial outcomes based upon an appropriate match between stressor and coping. Using the earlier example of the nurse in conflict with a nurse colleague regarding personal internet use in the operating room, the nurse may decide (through cognitive appraisal) the best way to handle the conflict is to directly confront the nurse to resolve the conflict. Or, given the characteristics of the situation, the nurse may decide that a more emotion-focused coping strategy such as seeking emotional social support from friends and family will produce better outcomes by reducing negative emotion.

On the other hand, ineffective or maladaptive coping efforts are not considered beneficial to the individual. Indeed, maladaptive coping refers to coping efforts that either do not produce an adaptational outcome or produce a detrimental outcome for the individual such as increased negative affect or loss of sleep (e.g., Kohler, Munz \& Grawitch, 2006; Parasuraman \& Hansen, 1987). For instance, using substances such as alcohol or illicit drugs as a frequent source of tension-reduction in response to stressors may lead to more severe problems in the long-term, such as alcoholism (e.g., Greeley \& Oei, 1999; Rehm, Taylor, \& Room, 2006). Maladaptive or ineffective coping is not restricted to substance use or any one type of coping, however, as any coping effort can fail to produce a beneficial outcome or generate a detrimental outcome. For example, adopting a problem-focused coping strategy by confronting the nurse colleague about the inappropriate internet use could potentially intensify the conflict with more resentment 
and negative emotion. Similarly, an emotion-focused coping approach such as avoidance of the nurse colleague or self-distraction to evade the problem may also exacerbate or prolong the conflict between them. Therefore, coping is considered effective or ineffective depending upon outcomes of the coping efforts for the individual in the specific context of the stressor.

In their review of empirical research on coping effectiveness, Zeidner and Saklofske (1996) identify eight primary criteria used to assess coping effectiveness. First, coping effectiveness can be determined based upon the resolution of the conflict or stressful situation since the coping efforts should be instrumental in removing or resolving the stressful encounter when possible. Reductions in physiological reactions or psychological distress are two indicators for coping effectiveness, as adaptive coping should have both physical and mental benefits for the individual (Pearlin \& Schooler, 1978). As coping should also affect social life aspects, normative social functioning is another criterion whereby coping effectiveness can be determined. Although coping effectiveness is certainly context-specific and a substantial change in daily behavior in relation to major stressors (e.g., loss of a job, death of a loved one) can denote effective coping, a return to pre-stress activities can also indicate coping effectiveness. Related to a few of these other criteria, the well-being of those affected by the stressor, including the self, a spouse, children or coworkers is another indicator of effective coping. Maintaining a positive self-esteem throughout the stressful experience is also commonly considered to be another appropriate gauge of whether the individual is effectively coping with the stressor. 
The final criterion for coping effectiveness put forth by Zeidner and Saklofske (1996) is perceived coping effectiveness. In other words, coping effectiveness is evaluated by directly querying how effective the coping was from the individual's own perspective. This approach allows the individual to report the effectiveness of the coping strategy based on their own evaluation. In many ways, perceived coping effectiveness may be the most appropriate indicator of coping effectiveness. For instance, since coping is profoundly influenced by cognitive appraisals, individual cognitive evaluations of effectiveness may be most consistent with stress and coping theory (Aldwin \& Revenson, 1987; Lazarus \& Folkman, 1984). Moreover, individual evaluations are often more powerful predictors of outcomes than the actual stressors themselves (Dewe, 1989) and truly objective criteria for coping effectiveness are seldom available (e.g., Zapf \& Gross, 2001). Therefore, consistent with the transactional model, if the individual believes or perceives a beneficial effect, it may be argued that the coping efforts were effective, regardless of any observed effect (Lazarus \& Folkman, 1984). As such, coping effectiveness may best be measured by the perceived coping effectiveness ratings of individuals coping with stressors in specific contexts, such as nursing. As this dissertation is focused on nurse coping effectiveness, I now discuss coping and coping effectiveness within nursing.

Coping in the nursing workplace context. As a profession with high levels of chronic work stress and many potential stressors, nursing is a context in which coping is a common occurrence. For instance, interpersonal stressors (e.g., WIC) are often unavoidable as they are frequently tied to the role of a nurse, forcing the nurse to cope 
with the stressful event (e.g., Duddle \& Boughton, 2007; Farrell, 2001; Leiter, 1991). Moreover, the healthcare context represents a workplace in which high job expectations (e.g., patient care) often produce elevated levels of perceived stress and require an adaptive coping response to mitigate the harm that could come from this high stress. As a result, numerous studies have been devoted to examining nurse stress and nurse coping (e.g., Chang et al., 2005; Dewe, 1993; Johnston, Beedie, \& Jones, 2006), particularly in efforts to identify those types of coping associated with good outcomes such as physical or mental health (e.g., Lambert et al., 2004; Xianyu \& Lambert, 2006).

In spite of this extensive literature on the subject of nurse stress and coping, the existing literature contains several conflicting results regarding the effectiveness of specific types of coping. For instance, Cheuk et al. (1997) suggests that problem-focused coping is more effective, since it is associated with improved outcomes in dealing with negative patient interactions. In this study, a sample of 212 nurses from Hong Kong was used to investigate whether recurrent rejection of offered help (spurning) was associated with nurse burnout and what types of coping were able to effectively mitigate this relationship. Mean difference analyses (i.e., two-way ANOVA) revealed that nurses who were more spurned experienced greater burnout than those who were less spurned. Moreover, those employing problem-focused coping experienced less burnout than those who used emotion-focused coping strategies. As such, these results suggest that problemfocused coping may be effective in dealing with nursing interpersonal stressors.

However, other research suggests that emotion-focused coping is effective. For example, using a sample of 59 full-time registered oncology nurses, Florio, Donnelly, and 
Zevon (1998) gathered perceived effectiveness ratings of different coping techniques in dealing with frequent nursing stressors (e.g., interpersonal issues at work). This study revealed coworker social support, positive reappraisal, and taking a growth perspective (forms of emotion-focused coping) were the most effective coping efforts in reducing overall nurse distress. Similarly, using objective records from the organization, an investigation of absenteeism from work as a coping mechanism (another form of emotion-focused coping), found nurse absenteeism to significantly predict lower work stress (Hackett \& Bycio, 1996). Elevated stress levels continued across work days when individuals came in to work, but decreased after taking a day off. This is consistent with literature suggesting that avoidance can be an effective means of dealing with physical and emotional fatigue associated with work in giving the individual a needed break or rest from the stressor (Carver et al., 1993). As such, these two studies represent studies within this literature that argue that emotion-focused coping is effective in dealing with nursing stressors. As such, the nursing literature contains conflicting results regarding effective coping strategies for nurses.

It should be noted that there is increasing evidence of consensus in the literature on one particular point: that substance use is a maladaptive emotion-focused coping strategy, including for nurses. Consistent with the tension-reduction hypothesis (Conger, 1956), alcohol and substance use has been identified as a maladaptive coping strategy, both contributing to and potentially becoming a substantial health problem in many other populations (e.g., Frone, 2008; Hyman \& Sinha, 2008; Mohr et al., 2001). For example, frequent use of substances to cope with stressors can develop into such problems as 
alcoholism or drug addiction, thus producing little to no beneficial outcomes and often becoming stressors themselves. Although there is evidence suggesting that nurse substance use to cope with stressors is not more prevalent than that found in the general population (Hughes, Howard, \& Henry, 2002), other studies have found that nurses are using substances to cope with nursing stressors (Trinkoff \& Storr, 1998; Trinkoff, Zhou, \& Storr, 1999; Trinkoff et al., 2000). For example, in a study examining responses of 3,600 nationally representative working nurses, Trinkoff et al. (2000) found that substance use was related to both workplace access and nurse role strain. As workplace access to substances increased and role strains increased, substance use also increased, suggesting that the nurses were using substances to cope with work stressors. Therefore, as nurses engage in substance use to cope with their work stressors, particularly over time, they are engaging in maladaptive coping behaviors that will likely produce future problems and challenges. Therefore, using substances to cope with nursing WIC was not considered as potentially adaptive in this dissertation.

Through application of the transactional model, many of the conflicting results regarding nurse coping effectiveness might be explained as a function of individual or situational factors such as cognitive appraisal or type of stressor. Accordingly, rather than attempting to identify one superior or most effective coping behavior, both problemfocused and emotion-focused coping could be considered effective forms of coping. As such, the conflicting results regarding nursing coping effectiveness would not be portrayed as inconclusive, but rather supportive of the transactional model's assertion that any type of coping can be considered effective, depending upon situational variables. 
Thus, the transactional model provides a framework that can explain these conflicting results within the nursing literature.

Furthermore, likely contributing to the nursing literature's struggle to identify effective coping techniques, many of these studies suffer from methodological limitations. Consistent with the approach in the general literature, a majority of nurse coping studies focus on identification of coping techniques used most frequently by nurses to cope with nursing stressors and measuring the associated outcomes, usually with cross-sectional designs (e.g., Bianchi, 2004; Lambert et al., 2004; Xianyu \& Lambert, 2006). Unfortunately, these studies can only examine correlations between general nurse stressors, coping frequency, and outcomes of interest (e.g., physical, mental health), precluding identification of coping strategies that are more effective, especially over time. Moreover, this approach does not allow an analysis of the effectiveness of different types of coping with specific types of stressors such as nursing WIC.

An additional methodological shortcoming in this literature is an almost exclusive focus on between-person differences. A between-persons perspective is useful in identifying differences based upon averages across all participants and observations, but is unable to explain important differences that may arise across multiple observations within individuals (Tennen \& Affleck, 1996). In other words, a between-persons approach cannot account for any variation that individual nurses exhibit in their coping across time. A within-persons perspective, however, does not suffer from these drawbacks (in the next chapter, I discuss this approach in more detail). Therefore, a research design based on the transactional model that is longitudinal, examines types of 
coping in relation to specific stressors (e.g., WIC), and incorporates a within-persons approach to investigating coping over time has the ability to address many of the methodological limitations and inconsistencies within the nurse stress and coping literature.

In summary, the transactional model provides a process model of work stress and coping that is comprehensive and well-supported in the literature. By using individual cognitive appraisals, the transactional model takes into account individual variation both between- and within-persons in coping responses to stressors. Moreover, the distinction between problem-focused and emotion-focused coping is both theoretically and empirically supported within the literature and provides an appropriate framework in which to classify coping behaviors. When evaluating the effectiveness of these coping efforts, the literature suggests that perceived coping effectiveness from the perspective of the individual may be the best approach. Finally, the nursing literature has been plagued with inconsistent results and methodological issues in examining coping effectiveness that can be resolved by applying the transactional model in a longitudinal research design using a within-persons approach that is focused on specific stressors. In the following chapter, I discuss the goodness of fit hypothesis as proposed in the transactional model (Lazarus \& Folkman, 1984), which provides specific predictions regarding coping effectiveness and is central to the purpose of this dissertation. 


\section{Chapter 4: The Goodness of Fit Hypothesis}

Based off of the transactional model, the goodness of fit hypothesis specifies coping effectiveness as a function of an appropriate match or "good fit" between cognitive appraisals of control and coping (Lazarus \& Folkman, 1984; see Figure 4). Under this hypothesis, no one type or set of coping strategies is hypothesized to be universally effective or ineffective (with the exception of substance use, as outlined previously), as the fit or match between control appraisals and the type of coping is depicted as the critical mechanism determining coping effectiveness. The goodness of fit hypothesis specifically states that problem-focused coping will be more effective under conditions of high perceived control and emotion-focused coping will be more effective under conditions of low perceived control (Lazarus \& Folkman, 1984). This is due to the theoretical proposition that control allows the individual to effect a change in the problem or stressor itself, but lack of control prevents these efforts, forcing the individual to adopt an emotional regulation approach. Empirical evidence further supports perceived control as a critical variable predicting coping outcomes (e.g., Compas, Banez, Malcarne, \& Worsham, 1991). Hence, a "good fit" between control appraisal and coping strategy is hypothesized to produce increased effectiveness of coping efforts or, in other words, adaptive outcomes.

Even as a successful match between control appraisal and coping is depicted as more effective, a mismatch between control appraisals and coping produce less effective or maladaptive outcomes. As a graphical representation of these hypothesized relationships, Figure 4 depicts effective or adaptive associations by solid lines and the 
ineffective or maladaptive relationships are represented by dotted lines. Starting with the stressor, solid lines lead from stressor to high control or low control appraisals, as neither high nor low control appraisal of its own accord is considered maladaptive. Tracing the first pathway, high control appraisals matched with problem-focused coping produce adaptive coping outcomes. The second pathway, on the other hand, denoted by a dotted line, demonstrates a mismatch of high control appraisals with emotion-focused coping as producing maladaptive outcomes. Similarly, the third pathway illustrates another maladaptive mismatch of low control appraisals with problem-focused coping. Finally, the fourth pathway demonstrates the hypothesized effective coping outcomes for the appropriate match of low control appraisals with emotion-focused coping. Based off of this graphical model, Figure 5 represents the specific hypothesized relationships in my model.

Using the example from the previous chapter regarding improper use of internet privileges for personal use in the operating room, the nurse will assess the amount of control the nurse has over the conflict and associated outcomes. If the nurse perceives high control, the nurse likely has the ability to successfully enact a problem-focused coping strategy such as directly confronting the offending coworker or by seeking the instrumental help of a nurse manager. Thereby, the conflict stressor can be eliminated, managed, or altered effectively, representing pathway 1 in Figure 4. Following the high control appraisal, if the nurse were to employ an emotion-focused coping strategy such as seeking emotional social support from other nurse coworkers, this strategy would likely not be as effective due to needless prolonging of the stressor (pathway 2 in Figure 4). On 
the other hand, if the nurse was to perceive low control in the conflict, the nurse does not likely perceive the ability to directly alter the conflict through direct confrontation or instrumental help of a supervisor, which can then exacerbate the problem (pathway 3 in Figure 4). However, under conditions of low control appraisal, seeking emotional social support from other nurse coworkers would be an effective way to regulate the negative emotional response, representing pathway 4 in Figure 4. In this study, I plan on testing, therefore, the hypothesized effective pathways, by examining pathway 1 and pathway 4 , as specified in Figure 5.

Although many studies have examined the goodness of fit hypothesis, goodness

of fit has not been directly tested in the workplace or among nurses, despite some preliminary evidence consistent with goodness of fit predictions. Moreover, the literature on the goodness of fit hypothesis emphasizes the importance of a proper methodological approach to accurately test goodness of fit predictions. Therefore, while discussing this literature, I focus on features of an appropriate examination of goodness of fit within the nursing workplace context.

\section{An Appropriate Approach to Examining the Goodness of fit Hypothesis}

Despite the intuitiveness of the goodness of fit hypothesis, the general literature has produced inconsistent results, as many studies have produced only partial support. For instance, several studies have only found support for problem-focused coping being more effective than emotion-focused coping under conditions of high appraised control (e.g., Masel, Terry, \& Gribble, 1996; Vitaliano, DeWolfe, Maiuro, Russo, \& Katon, 1990); whereas other studies support emotion-focused coping as more effective than 
problem-focused coping in situations of low appraised control (e.g., Carver et al., 1993; Zakowski et al., 2001). Some studies have suggested that these inconclusive results are due to contextual influences, such as a lack of variance in perceived control (e.g., Kendall \& Terry, 2008; Roberts, 1995). Others have identified methodological limitations of prior studies that may have hampered the ability to appropriately test the goodness of fit hypothesis (e.g., Park et al., 2001; Park et al., 2004). As such, this literature suggests that an accurate test of goodness of fit requires careful consideration of both context and method.

Workplace context. As a context within which a majority of adults spend a considerable amount of time (e.g., Hepburn, Loughlin, \& Barling, 1997) and levels of perceived control over job demands and stressors vary considerably (e.g., Karasek, 1979; Theorell, 2003), the workplace provides a good context for a test of the goodness of fit hypothesis. Although the literature lacks a test of the goodness of fit hypothesis in the workplace, there is some evidence providing tentative support. For instance, in a study on victims of workplace bullying, Zapf and Gross (2001) found problem-focused coping efforts (i.e., direct confrontation) were not an effective means of dealing with workplace bullying. Rather, those who successfully coped with the bullying were those who used more passive strategies such as avoidance of the bully or emotional social support (i.e., emotion-focused coping). Since workplace bullying is defined by a perceived power imbalance where the victim of bullying often perceives little personal control in the interaction (Salin, 2003), these results are consistent with the goodness of fit hypothesis. 
Other studies provide some additional support for the workplace as an appropriate context in which to test the goodness of fit hypothesis. When querying 86 part-time employed students regarding stressful conflicts at work, Hahn (2000) found those who identified with an internal locus of control and used problem-focused coping were able to effectively reduce their distress more than if they used other coping strategies. This is consistent with the goodness of fit hypothesis, though this study examined control as a personality trait that remains relatively constant (i.e., locus of control) rather than as a cognitive appraisal which varies between situations. In another study, Portello and Long (2001) discovered a significant positive relationship between conflicts with low control appraisals and the use of disengagement coping as well as a positive association between conflicts with high control appraisals and engagement coping in a sample of 157 female managers. Therefore, inasmuch as disengagement can be considered emotion-focused and engagement as problem-focused, these findings are consistent with the goodness of fit hypothesis. However, this study did not directly examine the effectiveness of coping in reducing subsequent distress, as the goodness of fit hypothesis specifies. Nevertheless, this study provides additional support for the goodness of fit predictions within the workplace.

The nursing literature also provides some evidence consistent with goodness of fit predictions. For example, in a cross-sectional study on nurse stress and coping, Tyler and Cushway (1992) administered questionnaires to 72 registered full-time nurses. Unexpectedly, they found that although avoidant coping strategies were generally associated with poor mental health outcomes, problem-focused and emotion-focused 
coping (including a few items of avoidance coping) produced both improved and decreased overall health. As an interpretation of these findings, they suggest that both problem-focused and emotion-focused coping may be appropriate under certain situational conditions and that the use of inappropriate types of coping may actually increase nurse stress and strain. Later studies identified perceived control over nursing stressors as a very influential factor in the stress and coping process (e.g., Boey, 1999; Fox, Dwyer, \& Ganster, 1993), suggesting that perceived control may be one of these situational conditions. Therefore, taken together, these studies within the nursing literature suggest that the nursing workplace is an appropriate context in which to test the goodness of fit hypothesis.

Methodological considerations. In order to appropriately test the goodness of fit hypothesis, the literature has demonstrated that, in addition to context, certain methodological characteristics must be taken into consideration. Indeed, although the general literature contains inconsistent results for the goodness of fit hypothesis, certain studies have found full support for both predictions of the hypothesis (e.g., Compas et al., 1988; Forsythe \& Compas, 1987; Park et al., 2001; Park et al., 2004). Improving the ability to test goodness of fit, these studies have two unique methodological characteristics in common: a focus on stressors that vary in perceptions of control and a within-person approach examining multiple stressors.

First, studies fully supporting the goodness of fit hypothesis often focus on social stressors that have substantial variation in perceived control. Whereas many of the studies finding little or no support for goodness of fit have examined physical health stressors 
that may show little fluctuation in control (e.g., Kendall \& Terry, 2008), supporting studies focus on other stressors, such as a recent most distressing negative event or interpersonal stressors (e.g., Compas et al., 1988). For example, in early support of the goodness of fit hypothesis, Forsythe and Compas (1987) used a cross-sectional design to query college students about their coping responses to the most distressing event they had experienced in the recent past. Those who used problem-focused coping for stressors perceived to be controllable reported fewer psychological symptoms (e.g., depression, anxiety) than those who used similar coping efforts for stressors of low control. When emotion-focused coping was used to deal with stressors of low perceived control, fewer psychological symptoms developed as opposed to conditions of high control. Hence, this study provided full support for the goodness of fit hypothesis by examining stressors other than physical health stressors, which is likely a more appropriate test of goodness of fit.

Another study further illustrates the importance of examining stressors other than physical health stressors. Using a cross-sectional design with 130 adolescents, Compas et al. (1988) found that adolescents varied their coping strategies according to the controllability of interpersonal stressors. Those adolescents who successfully matched controllable interpersonal stressors with problem-focused coping developed less subsequent emotional and behavioral problems, as indicated by their parents. However, those who employed emotion-focused coping for controllable interpersonal stressors were more likely to develop emotional and behavioral problems. As suggested by the results of this study, the successful pairing of control appraisal with coping efforts 
according to the goodness of fit hypothesis seems effective for interpersonal stressors. More importantly, the focus on interpersonal stressors likely enhanced the study's ability to garner full support for goodness of fit due to increased variability in control appraisals (Compas et al., 1988). Thus, these studies suggest a focus on social stressors may improve the ability to test the goodness of fit hypothesis, likely due to variation in control appraisals.

Second, most previous studies have produced inconclusive results by examining goodness of fit from a between-person perspective in relation to a single stressor. Those studies taking a within-person perspective using multiple assessments, however, have produced much more supportive evidence. This approach offers strong advantages to testing the goodness of fit hypothesis including assessment of coping effectiveness for each particular coping occasion using coping type and control appraisals within-persons; and goodness of fit style, wherein individuals can be distinguished in how well they follow goodness of fit across coping occasions (Park et al., 2004). Therefore, using this approach allows examination of the usual between-person effects using averages across the entire sample while simultaneously permitting investigation of within-person effects over multiple coping occasions for each individual.

In one such study, Park et al. (2001) found considerable evidence supporting the goodness of fit hypothesis. Using longitudinal data gathered in 12 bimonthly interview occasions from a sample of 314 men, some of whom were caregivers of HIV+ men and others who were HIV+ men, Park et al. (2001) analyzed the data using multilevel regression techniques consistent with a within-person perspective. Specifically, they 
reported planful problem solving (i.e., problem-focused coping) used to cope with stressors of high perceived control as significantly predictive of less depressive mood over time. Additionally, distancing (i.e., emotion-focused coping) used to cope with stressors of low perceived control was marginally significantly predictive of less depressive mood over time. In full support of the predictions of goodness of fit, this study demonstrated that within-person matching of coping and appraisal was related to coping effectiveness. Hence, this study provides some groundbreaking evidence of the enhanced testing ability a within-person approach has to offer in relation to the goodness of fit hypothesis.

In a similar study taking a within-person approach using Internet daily diary methodology, Park et al. (2004) reported results consistent with goodness of fit. Using multilevel modeling techniques to analyze data gathered from a sample of 190 undergraduate students, they investigated the effects of coping and control on daily mood over the course of 28 days. As predicted by the goodness of fit hypothesis, they reported a significant relationship between level of perceived control over stressors and type of coping in predicting coping effectiveness. First, problem-focused coping was more likely to be used under high control appraisals and emotion-focused coping was more likely under low control appraisals. Second, when examining coping outcomes, the association between problem-focused coping and daily positive mood was stronger when dealing with high control stressors opposed to low control stressors, directly confirming the goodness of fit hypothesis. Although the relationship between emotion-focused coping and low control did not significantly predict average improved mood across the entire 
sample (between-persons), a subset of the participants experienced substantially improved mood, providing tentative support for this prediction as well. Or, in other words, goodness of fit style varied across individuals (i.e., variance components or slopes were significant), suggesting that some were "better fitters" and others were "poorer fitters" (within-persons). Therefore, the results of both of these studies fully support the goodness of fit hypothesis and suggest that a within-person perspective is the most appropriate approach to adopt when testing goodness of fit.

Finally, as this study provides the closest template for my own test of the goodness of fit hypothesis, I describe two additional details of the study as they relate to my measures (see Measures section in Chapter 6). First, in order to assess coping, Park et al (2004) used 12 representative items from the subscales of the Brief COPE (Carver, 1997), which is based off the COPE measure created to comprehensively represent coping from theoretical reasoning (Carver et al., 1989). Park et al. (2004) selected the 12 highest loading items on each of the higher order factors of active problem solving, social support/emotion-focused coping, acceptance/restraint, and disengagement/denial from the original COPE measure (Carver et al., 1989). In order to investigate which of these subscales represented problem-focused and emotion-focused coping, they conducted an Exploratory Factor Analysis, which produced three interpretable coping factors: problemfocused, emotion-focused, and avoidance. Specifically, six items represented emotionfocused coping (e.g., getting emotional support from others), three items signified problem-focused coping (e.g., taking action to make the situation better), and three items for avoidance coping (e.g., giving up trying to deal with it). As such, the approach of 
Park et al. (2004) suggests that the Brief COPE can be used to test the goodness of fit hypothesis.

Second, as potential moderators of goodness of fit, Park et al. (2004) investigated two specific personality (person-level) characteristics: neuroticism and optimism. Results showed that neuroticism was a significant moderator of both the emotion-focused coping/control and the avoidance coping/control associations in predicting overall mood. Optimism, on the other hand, was a significant moderator for the problem-focused coping/control association in predicting overall mood. These results provide evidence of the moderating effects of personality characteristics on goodness of fit. As substantial variance between individuals in how well they follow goodness of fit has emerged (i.e., goodness of fit style) alongside evidence of individual differences (i.e., personality characteristics) moderating goodness of fit predictions (Park et al., 2004), other individual difference variables could also play a similar role. Therefore, in the next section, I discuss occupational tenure as a specific individual difference variable that may similarly moderate goodness of fit within the nursing workplace context.

In summary, two important methodological characteristics have been influential in determining full support for the goodness of fit hypothesis. First, whereas many studies have examined the goodness of fit hypothesis in relation to a physical injury or disease (e.g., Carver et al., 1993; Felton \& Revenson, 1984), studies finding the most support for goodness of fit have focused on stressors which are more likely to fluctuate in perceived control, such as social stressors. This is particularly important as little variance in perceived control limits the ability to accurately test goodness of fit (Kendall \& Terry, 
2008). Second, the within-person approach used by Park et al. (2001) and Park et al. (2004) has produced full support for goodness of fit and has important implications for future studies examining goodness of fit. For instance, both used a rigorous hierarchical linear regression technique to examine a longitudinal dataset. By so doing, the researchers examined both the within- and between-person effects of perceived control and coping on mood, enabling an exploration of whether the predictions of the hypothesis were accurate for some individuals (i.e., slopes) rather than relying upon averages across the whole sample. Therefore, by adopting a similar approach of examining coping in relation to stressors (i.e., WIC) that fluctuate in perceived control using a within-persons perspective to examine multiple stressor events, I expected to produce similar confirming evidence of the goodness of fit hypothesis within the workplace context.

\section{Occupational Tenure}

A common limitation of the literature investigating the goodness of fit hypothesis is a general lack of attention to individual differences that may affect control appraisals and the type of coping used. Indeed, Park et al. (2004) discovered that some individuals' observations were more consistent with the predictions of the model than others, suggesting that there are likely individual difference variables that influence the goodness of fit model by moderating the relationship between control appraisal and coping. Within the workplace, and nursing in particular, tenure may be one such variable. Often used interchangeably, two conceptualizations of job tenure exist within the literature, including the amount of time spent within the occupation itself (i.e., occupational tenure) and the length of time employed by a specific organization (i.e., organizational tenure). By 
definition, occupational tenure is a more inclusive variable (i.e., represents time spent within previous organizations in the profession as well as within the current organization) and is used more frequently than organizational tenure within the nursing literature (e.g., Lambert et al., 2004). Therefore, for the purposes of the hypotheses of this dissertation, I focused on occupational tenure. In the next section, I will detail the evidence for occupational tenure as a moderator of the goodness of fit hypothesis followed by a discussion of the literature supporting occupational tenure as a predictor of coping choice. Throughout this section, I focus on the implications of such evidence for nurse stress and coping.

\section{Occupational tenure as a moderator of the goodness of fit hypothesis.}

Occupational tenure, representing one's prior work experience, can profoundly affect one's ability to match perceived control over stressors appropriately with coping type and, thus affect subsequent coping effectiveness as predicted by the goodness of fit hypothesis. Figure 6 illustrates this proposed relationship with occupational tenure modeled as a moderator of the individual's matching of perceived control with coping type. Specifically, this relationship is depicted as four bold arrows, representing the influence of occupational tenure on each of the four potential pathways of goodness of fit at the point of pairing perceived control and coping choice. Hence, occupational tenure influences the individual's ability to select coping efforts based on perceived control and can lead to either adaptive or maladaptive coping outcomes. It is important to note that as an individual difference variable, occupational tenure could potentially affect perceptions of control as well as coping choice. However, to be consistent with the goodness of fit 
hypothesis, occupational tenure is depicted as affecting the ability to appropriately match perceived control with coping type (see Figure 6).

Although no study has directly examined the effect of occupational tenure on the predictions of the goodness of fit hypothesis, there is some evidence to support this model. Specifically, a few studies suggest that as tenure increases, the ability to pay attention to appropriate environmental cues is enhanced, enabling the individual to cope more effectively with stressors (e.g., Moser \& Galais, 2007; Rotondo, 1999). For example, in a recent study, Moser and Galais (2007) provide evidence of a potential attenuating influence of tenure on the relationship between control and coping. In a crosssectional study using a total sample of 175 sales insurance agents, they found selfmonitoring to be positively correlated with job performance for those who have less tenure, but not for those with greater tenure. As high self-monitors are typically more concerned with impression management and behave inconsistently across situations (see Knight, 2002), they may be so preoccupied with making a good impression on others that they miss important environmental cues and are thus unable to cope effectively. Although this study looked at organizational tenure rather than occupational tenure, the results of this study suggest that as tenure increases, the individual is better able to focus on characteristics of stressors, appraise their control more accurately and adapt their coping strategies accordingly.

Similarly, Bradley (2007) found further supportive results when investigating the moderating role of tenure on the relationship between job stressors and strain in the workplace. Using a sample of 422 experienced (greater occupational tenure) and 248 
beginning schoolteachers (less occupational tenure) comprised of 498 (74\%) female teachers, this prospective study examined group differences in managing job demands and outcomes eight months later. At follow-up, a significant difference emerged between the groups in intention to quit and job dissatisfaction, as those with less tenure had poorer outcomes than their tenured counterparts. Moreover, those with less tenure reported more benefits from increases in perceived control than those with more tenure. Although this study did not specifically examine the relationship between perceived control and coping, the results tentatively suggest those with more tenure are able to cope better in situations of varying control. Or in other words, those with more tenure have likely learned to not rely solely upon high control to cope effectively, but discovered ways to vary their coping efforts to match conditions of low control.

Another study focusing exclusively on sexual harassment of women employees in the workplace produced further evidence consistent with the model. Specifically, Cortina and Wasti (2005) found that women with more occupational tenure successfully avoided sexual harassment situations more often than those with less occupational tenure. As a potential interpretation, the researchers suggested that increased occupational tenure was a protective factor for the women employees, as prior knowledge or experience led them to focus on important environmental cues when appraising the situation and successfully match these with the best coping technique. As a workplace stressor, sexual harassment is often considered one where the victim has little to no control in the interaction (Cortina \& Wasti, 2005) and, according to the goodness of fit hypothesis, an emotion-focused coping strategy such as avoiding the situation should produce more effective outcomes. As such, 
this study supports the notion that increased occupational tenure likely enables workers to cope more effectively by appropriately pairing control appraisals with coping efforts.

Within the nursing literature only minimal evidence supportive of this model is available, primarily due to the fact that very few studies have investigated occupational tenure, control and coping. However, two qualitative studies suggest that occupational tenure may help improve coping outcomes. For instance, among 10 nurses Gotay, Crockett, \& West (1985) found those nurses who had less tenure were more stressed than their tenured counterparts, possibly due to challenges in coping effectively with nurse stressors. Moreover, Duddle and Boughton (2007) reported that those nurses who had more occupational tenure were better able to cope with interpersonal conflicts that erupted between nurses. Tenured nurses detected the signs that a nurse colleague was under extreme amounts of stress, recognized their control of the situation and adjusted their coping responses to handle conflicts more effectively than novice nurses. Of course, these qualitative nursing studies are limited in providing much evidence for the model proposed in Figure 6, but they provide initial justification and support for the testing of this model among nurses. That is, that problem-focused coping used in high control situations and emotion-focused coping used in low control situations will be even more effective for those with greater tenure than those with less tenure.

Occupational tenure as a predictor of coping. Within the very limited amount of literature on the subject, the direct relationship between occupational tenure and coping frequency remains to be explored using a within-person approach. Indeed, most of the studies producing evidence of occupational tenure directly affecting coping frequency 
have relied upon cross-sectional designs and use between-person perspectives (e.g., Havlovic \& Keenan, 1995), sometimes producing conflicting evidence (e.g., Decker \& Borgen, 1993). However, this literature generally supports a positive relationship between tenure and problem-focused coping as well as a negative relationship between tenure and emotion-focused coping (e.g., Havlovic \& Keenan, 1995; Welbourne, Eggerth, Hartley, Andrew, \& Sanchez, 2007; Wright \& Bonett, 1993). For instance, in their cross-sectional study examining coping frequency in supervisory level positions, Havlovic and Keenan (1995) reported that occupational tenure was negatively related to help-seeking (i.e., emotion-focused coping). As such, this study tentatively suggests that as tenure increases, employees are more likely to rely upon strategies other than emotionfocused coping such as problem-focused coping efforts.

Other studies provide similar findings. In a cross-sectional study examining the effects of occupational tenure on work stress and coping for career females, Pandey and Srivastava (2003) used a sample of 240 women in teaching, banking, and railway occupations. Occupational tenure was split into two categories with short tenure being less than five years and long occupational tenure being greater than five years to predict the relationship between coping strategies, work stress (i.e., interpersonal) and physical health. Short occupational tenure was positively predictive of increased use of restraint coping and focus on and venting of emotions (both emotion-focused coping), whereas long occupational tenure did not. As such, this study suggests that emotion-focused coping is used more by those with less tenure and problem-focused coping is used more by those with increased tenure. Moreover, in another cross-sectional study, Lu, Kao, Siu, 
and Lu (2010) provide additional evidence of the relationship between problem-focused coping and tenure using a self-report survey of 360 Chinese employees, though this analysis used organizational tenure rather than occupational tenure. Hierarchical linear regression analyses revealed both occupational tenure and active coping strategies (i.e., problem-focused coping) as positive significant predictors of job performance (e.g., quantity of work, quality of work, job knowledge). Therefore, this study suggests that as both quantity and quality of work increases relative to tenure, problem-focused coping becomes more frequent.

Additional evidence supports the connection between problem-focused coping and tenure for nurses as well. In a dissertation examining coping with workplace stressors and organizational outcomes, Sundberg (2003) used a sample of 157 direct care nurses and examined the link between occupational tenure and coping strategies (i.e., proactive, avoidance). Those who had been employed more years as a nurse were less likely to miss days of work, a common avoidance coping strategy (Hardy et al., 2003). Therefore, the emotion-focused coping strategy of avoiding work was used less by those with more occupational tenure. Although this study did not examine problem-focused coping directly, the negative relationship between emotion-focused coping and tenure suggests that other types of coping, such as problem-focused coping, were being used.

Welbourne et al. (2007) found further evidence of a positive relationship between tenure and problem-focused coping frequency. In this study, a total of 190 registered nurses and licensed practical nurses provided self-report responses regarding their coping styles, job satisfaction, and occupational tenure. When regressing job satisfaction on 
coping styles and occupational tenure, both tenure and problem-focused coping emerged as positive significant predictors. Therefore, this study also supports the assertion that problem-focused coping and tenure are positively related. Finally, in a cross-sectional study using 310 full-time nurses in Japan, Lambert et al. (2004) examined occupational tenure in relation to nursing stressors and coping efforts. Problem-focused coping efforts (problem-solving) emerged as significantly positively related to occupational tenure whereas emotion-focused coping (i.e., accepting responsibility, seeking social support) was significantly negatively associated with occupational tenure.

Therefore, depending almost completely on cross-sectional designs and a between-person perspective, the nursing literature and the general literature assert that tenure is positively associated with problem-focused coping frequency and negatively associated with emotion-focused coping frequency. However, this relationship has yet to be examined using a within-person approach, which has been established as the most appropriate way to investigate the stress and coping process (Lazarus, 2006). Moreover, this approach can specifically identify whether this relationship also varies within individuals while addressing the need for additional research in the very limited literature on the effects of occupational tenure in the stress and coping process. Figure 7 depicts my hypothesized relationship between occupational tenure and coping frequency.

Finally, another plausible explanation for the overall association of coping frequency with occupational tenure may be due to an expanding coping repertoire over time (see Figure 7). That is, as an employee gains more experience within an occupation, their available coping mechanisms may expand to include other coping efforts not 
previously available. Similar processes have been noted within children and adolescents, where new coping responses are learned over time and are then used to deal with future stressors (e.g., Compass et al., 1988; Skinner \& Zimmer-Gembeck, 2007). Despite the intuitiveness of this explanation, this has yet to be examined within the workplace and represents another gap within the literature. As such, this is an important research question that can be explored in the current study.

In summary, the goodness of fit hypothesis specifies that problem-focused coping is most effective when used to cope with stressors of high perceived control and emotionfocused coping is most effective when paired with stressors of low perceived control (Lazarus \& Folkman, 1984). Although the general literature has produced mixed results for these predictions, an examination within the nursing workplace using a withinpersons perspective of coping with WIC is an appropriate approach to testing goodness of fit. Moreover, the literature has yet to explore certain contextual variables that may moderate the goodness of fit model, such as occupational tenure. In particular, evidence suggests that as occupational tenure increases, employees' ability to cope effectively with work stressors of differing levels of control is enhanced (e.g., Bradley, 2007; Cortina \& Wasti, 2005). Moreover, consistent with the literature, occupational tenure may also be an important predictor of coping frequency (e.g., Havlovic \& Keenan, 1995), but the examination of this relationship has been primarily restricted to cross-sectional designs employing a between-persons approach. As such, a longitudinal design using a withinperson approach provides both an appropriate approach to testing goodness of fit while 
offering further insight into the effect of occupational tenure on goodness of fit and coping frequency. 


\section{Chapter 5: Study Purpose and Hypotheses}

Although the goodness of fit hypothesis has been examined within several contexts, the literature, to the best of my knowledge, lacks a test of the goodness of fit hypothesis within the workplace context and, more specifically, among nurses. Moreover, the literature clearly demonstrates the necessity of conducting an appropriate test of goodness of fit by examining multiple stressors that vary in perceived control (e.g., social stressors) using a within-persons perspective (e.g., Park et al., 2004). With this in mind, the goal of this dissertation was to apply the theoretical propositions of the goodness of fit hypothesis within the nursing workplace context in relation to workplace interpersonal conflict using a within-persons approach. As such, this dissertation offers the unique contribution of providing the first examination of the goodness of fit hypothesis in the nursing context relative to significant social stressors.

To accomplish this purpose, this dissertation employed multilevel modeling techniques to analyze data comprised of twelve weekly within-persons observations. This methodology has been identified as the appropriate technique whereby to examine the coping process (Lazarus, 2006) as well as test goodness of fit (Park et al., 2004). Since previous research using this methodology has identified significant variation between individuals for goodness of fit (Park et al., 2001; Park et al., 2004), this dissertation also investigated a moderator that may explain this variation. Specifically, as a factor that influences perceptions of the environment and affects the individual's ability to pair perceived control with coping type (e.g., Cortina \& Wasti, 2005; Moser \& Galais, 2007), occupational tenure was examined as a moderator of goodness of fit. Moreover, the 
literature suggests that occupational tenure and coping frequency are related (e.g., Lambert et al., 2004), but this conclusion has been primarily based upon results from cross-sectional designs employing a between-persons approach. Hence, the current study provides further unique contributions by exploring occupational tenure as a moderator of the goodness of fit hypothesis and as a predictor of coping frequency using a longitudinal design from a within-persons perspective among nurses.

\section{Hypotheses}

Goodness of fit hypothesis. As specified under the goodness of fit hypothesis (Lazarus \& Folkman, 1984), two predictions regarding coping effectiveness are explicitly detailed. First, problem-focused coping will be most effective when used to cope with stressors of high appraised control. Second, emotion-focused coping will be most effective when used to cope with stressors of low appraised control. Although support for these propositions in the literature is mixed (e.g., Conway \& Terry, 1992; Zakowski et al., 2001), most of these studies provide limited tests of goodness of fit by taking a between-persons perspective of coping using only one stressor. On the other hand, those studies providing the most support for these predictions have examined more than two stressors and adopted a within-person approach (Park et al., 2001; Park et al., 2004). Therefore, consistent with these latter studies, I included multiple social stressors (i.e., workplace interpersonal conflict) for nurses in a within-person design and expected to find support for both tenets of the goodness of fit hypothesis (see Figure 5).

Hypothesis 1a: Problem-focused coping will be rated as more effective on weeks when perceived control is higher versus weeks when perceived control is lower. 
Hypothesis 1b: Emotion-focused coping will be rated as more effective on weeks when perceived control is lower versus weeks when perceived control is higher.

Occupational tenure as moderator of the goodness of fit hypothesis. Although

this literature has demonstrated support for goodness of fit across individuals, significant individual differences have also emerged, suggesting that goodness of fit may demonstrate stronger associations for certain individuals. As a potential explanation for this, Park et al. (2004) suggest that certain moderating variables may be responsible for these differences. Within the workplace, one such variable may be tenure, as increases in occupational tenure (i.e., occupational) often lead to improvements in coping effectiveness, likely due to experience in coping with work stressors (Cortina \& Wasti, 2005). As such, I predicted that occupational nursing tenure would moderate the hypothesized relationships specified by the goodness of fit hypothesis. Specifically, those nurses with more tenure would exhibit improved effectiveness from matching coping efforts with appraised control than those with less tenure (see Figure 6).

Hypothesis 2a: The problem-focused coping-high perceived control relationship (i.e., Hypothesis 1a) will be stronger for nurses with greater occupational tenure relative to nurses with less occupational tenure.

Hypothesis 2b: The emotion-focused coping-low perceived control relationship (i.e., Hypothesis 1b) will be stronger for nurses with greater occupational tenure relative to nurses with less occupational tenure.

Occupational tenure main effect on coping frequency. Even as tenure likely interacts with the predictions of the goodness of fit hypothesis, the literature suggests that 
occupational tenure has main effects on coping frequency. Specifically, there is evidence suggesting that as tenure increases, problem-focused coping becomes more frequent and emotion-focused coping decreases (Lambert et al., 2004). As such, I predicted as nursing tenure (i.e., occupational) increases, frequency of problem-focused coping with interpersonal conflicts at work would increase. Moreover, I expected emotion-focused coping with interpersonal conflicts at work to decrease as nursing tenure increased. Finally, as a potential alternative explanation, I examined the association between occupational tenure and coping frequency variance (i.e., standard deviation), to explore changes in available coping mechanisms (see Figure 7).

Hypothesis 3a: Nurses with greater occupational tenure will engage in more problem-focused coping across study days in response to workplace interpersonal conflicts compared to nurses with less occupational tenure.

Hypothesis 3b: Nurses with less occupational tenure will engage in more emotion-focused coping across study days in response to workplace interpersonal conflicts compared to nurses with greater occupational tenure.

Research Question 1: Is occupational tenure related to changes in coping frequency variance (i.e., coping frequency standard deviations)?

In summary, I anticipated this dissertation would produce evidence supportive of both predictions of the goodness of fit hypothesis. Based off of the literature's suggestion, I also expected tenure (i.e., occupational) to interact with these predictions, such that as tenure increased, coping effectiveness as predicted by the goodness of fit hypothesis would also increase. Finally, I expected occupational tenure to have a main 
effect on coping frequency, so that greater tenure would be associated with increased problem-focused coping frequency and less tenure would be associated with increased emotion-focused coping frequency. 


\section{Chapter 6: Method}

This dissertation comprises a secondary data analysis of a larger project, the Oregon Nurse Retention Project (ONRP), which was directed by Robert R. Sinclair, Ph.D. (PI) and Cynthia D. Mohr, Ph.D. (co-PI) and supported by grant funding from the Northwest Health Foundation (Grant \# 14180) and from the National Institute for Occupational Safety and Health (T01 OH008435-02). The ONRP was a collaborative effort between Portland State University and the Oregon Nurses Association (ONA) and involved a multi-method approach in efforts to identify key factors in the retention of nurses in their current employment and within the nursing workforce (Sinclair et al., 2009). Data collection involved three distinct phases including a baseline survey assessment, a weekly survey study spanning 12 weeks, and a follow-up survey assessment (see Figure 8). The data for my dissertation comprised the weekly survey data and, given the number of acronyms that come up in this chapter, I once again refer the reader to the glossary of acronyms (see Appendix A).

As a research assistant in the ONRP, I was actively involved in all phases of this project, from the initial planning stages to dissemination of results. Throughout the project, I participated in weekly team research meetings (which eventually became monthly meetings as the project neared completion). At these meetings, I assisted in discussions of research design methodology, developing a conceptual framework to guide data collection, and constructing the survey instruments. Before data collection, I spearheaded the study IRB approval process by preparing documentation and submitting it to the Human Subjects Research Review Committee at Portland State University. I 
conducted a qualitative analysis of archival nurse staffing request documents in developing a measure of nurse staffing for the project and I assisted several nurse focus groups to ensure that our survey content and measures were relevant to nursing. I also participated in direct participant recruitment for the study and maintaining a good relationship with ONA by attending ONA conventions over the course of three years.

I also actively managed administration of the weekly survey and handled the online weekly survey database, where participant data were stored. I managed our participant survey response database, where I kept records of each survey that was completed. When there were missed surveys, I sent out email reminders to participants to encourage them to complete the next weekly survey. I provided additional technical assistance to participants who experienced difficulty with the online submission process. At the end of the weekly data collection, I conducted a raffle drawing and assisted in distributing these incentives and study compensations to participants.

Following the PI's move to Clemson University, I became the lead graduate student for the study at Portland State University, where I took on additional responsibilities. I directly handled participant inquiries regarding the study (by email and by phone) as well as managed important data and documentation issues (e.g., data cleaning, IRB). Once data collection was complete, I was actively involved in disseminating our research findings. First, I assisted in the preparation of the final technical report for the project, which was submitted to the funding agency. Second, I created a report summarizing these technical findings for nurse participants to view online. Third, as a Master's thesis project, I analyzed qualitative data from the weekly 
survey on interpersonal conflict between nurses to identify and characterize themes of these conflicts (Wright, 2009). Fourth, in hopes of benefiting nurses further, I produced a technical report of my Master's thesis findings, which I posted online for nurse participants to review. Finally, I have presented findings from the ONRP at several research conferences, both nationally and internationally ${ }^{1}$. As such, I have been intimately involved in the ONRP, providing instrumental assistance in research design, measure development, data collection, and interaction with community partners and participants.

\section{Participants}

Participation in this study was completely voluntary and participants received monetary compensation for their participation ( $\$ 15$ for baseline survey, $\$ 10$ for follow-up survey). In order to obtain a sample comprised of practicing registered nurses diverse in their occupational tenure and representative of rural, suburban, and urban acute-care facilities across Oregon, sample recruitment was conducted in a multi-step procedure.

${ }^{1}$ I assisted or gave the following presentations on ONRP data:

Sears, L.E., Cadiz, D., Wright, R.R., \& Sinclair, R.R. (2010, April). Sources and frequency of incivility versus support: What matters most? Paper presented at the annual meeting of the Society for Industrial and Organizational Psychology. Atlanta, GA.

Wright, R.R., Mohr, C.D., \& Sinclair, R.R. (2010, January). Evaluation of the interpersonal conflict construct: Implications for measurement. Poster presented at the annual meeting of the Society for Personality and Social Psychology. Las Vegas, NV.

Wright, R.R., Mohr, C.D., \& Sinclair, R.R. (2009, November). Evaluation of the workplace interpersonal conflict construct using a nurse sample. Paper presented at the annual Work, Stress \& Health conference. San Juan, Puerto Rico.

Deese, M.N., Sears, L.E., Sinclair, R.R., Wright, R.R., Cadiz, D. M., Jacobs, L.J., Mohr, C.D., \& Davidson, S.B. (2009, April). Bad vs. good: Do positive work events predict nurses' engagement? Poster presented at the annual meeting of the Society for Industrial Organizational Psychology Conference. New Orleans, LA.

First, researchers from the ONRP directly solicited nurses for their participation by attending a nursing convention provided by their professional organization (ONA). At 
this convention, researchers provided a detailed informational display where nurses were given specific information regarding the basic aims of the project and invited to participate. Second, nurses were solicited to participate through informational advertisements and newsletters circulated by ONA. This included an additional postcard mailing, which contained particular instructions and information such as when the study would begin and how interested nurses could participate. Although the primary recipients were members of ONA (i.e., nearly the entire sample), other nurses expressed interest (e.g., informed by their nurse colleagues) and were able to participate in the study.

Third, throughout the recruitment process, nurses were invited to register on a website constructed specifically for the project. Upon registration, nurses gave their consent to be contacted for participation in the study by the researchers. Finally, the nurse participants who had registered on the website were contacted via email or postal mail, according to their indicated preference, in order to obtain their consent to participate in the study. Out of the 428 nurses who participated in the larger study (baseline assessment survey), a subset of 148 nurses consented to additional participation in the weekly survey encompassing twelve weeks (see Appendix B), comprising the sample of the current study. All of the 148 nurse participants agreed to complete electronic versions of each weekly survey online.

The weekly survey sample was predominately female (93\%) and the average age of participants was 45 years $(S D=10.75)$. These particular sample characteristics were representative of 2008 ONA membership data (the same year the data for the current study was collected), which I obtained directly from ONA researchers for comparison. A 
total of $91 \%$ of the sample identified themselves as White, but this demographic could not be compared to ONA membership since ONA does not collect this information. Average occupational tenure of the sample, however, was 17 years $(S D=12.10)$, whereas the average occupational tenure of the ONA membership is substantially lower $(M=$ 9.92, $N=9,849$ ), suggesting that the sample lacked early career nurses. Sixty-five percent of participants were full-time nurses, $70 \%$ indicated their highest level of education to be a 4-year bachelor's degree or greater, and mean organizational tenure with their current organization was 11 years $(S D=8.76)$. Average time worked per week across the entire sample was about 37 hours $(S D=9.91)$. Preliminary analyses on all of these variables indicated no significant differences between the larger study participants and the weekly survey sample, suggesting there was no systematic participant selection bias in the weekly survey sample. Four participants, who consented to participate, opted out of the study (i.e., requested to discontinue their participation due to situational or scheduling constraints) before the end of the 12-week study period. One participant provided no conflict data and was therefore not included in subsequent analysis $(n=143)$.

\section{Procedure}

Nurse participants who expressed interest in participating in the weekly survey study were contacted via email, whereupon participants were asked to complete the first of twelve weekly assessment measures regarding their current primary work (many participants indicated they had two nurse jobs). This email message contained a link to an electronic copy of the measures, allowing them to access the surveys online. Each Sunday at noon, an automatically generated email reminder was sent to the participants 
containing similar links to each week's survey whereupon participants were only able to complete that week's survey one time. Participants were given a 48-hour time period during which to complete the online survey, which closed on Tuesday at noon. This specific time period was chosen to maximize participation, given the nature of their work (i.e., according to ONA, nurses were typically least busy on these days). Participants were unable to complete missed surveys or access their own previously submitted surveys (i.e., surveys from prior weeks). Subsequent weekly assessments were handled similarly, with an automatically generated email reminder asking participants to complete the web-based instruments each week by Tuesday at noon.

A comprehensive set of instructions on how to complete these measures accompanied each online survey. Additionally, participants were provided researcher contact information in case questions or concerns arose. Incentive for participation included monetary compensation based upon the number of surveys completed (i.e., $\$ 5$ per survey). Further, those participants who completed consecutive surveys for each four week period (e.g., weeks 1 through 4) were eligible for a raffle drawing for cash prizes (i.e., one of four $\$ 50$ prizes). In cases where two or more consecutive weekly surveys were not completed, another email reminder was sent by the researchers, encouraging participants to complete the next week's survey. Missing survey data was minimal, as few surveys were not fully completed by the participants (see Missing Data section in Chapter 6). Appendix B displays the informed consent along with the specific items from the weekly survey I used for this dissertation.

\section{Measures}


Tenure variables. Occupational tenure and organizational tenure were assessed using single items as part of the demographic information collected when participants registered online for the study. Occupational tenure was assessed by asking participants to indicate how long they had been a registered nurse and organizational tenure was obtained by asking how long they had been working in their current organization. Both were in a free-response format, but participants were prompted to respond in number of years. Within the literature, single item measures of self-report data such as occupational tenure are acceptable (e.g., Wanous et al., 1997).

Weekly workplace interpersonal conflict. Each week, participants were asked to "think about the most negative/stressful interpersonal interaction/conflict you had in the past 7 days with someone at your primary job." Participants were then asked to provide a brief description of this event, including causes of the conflict. If more than one conflict occurred during the past seven days, participants only reported the most negative or stressful. Rather than measure WIC as a psychological construct composed of different characteristics (e.g., Schieman \& Reid, 2008; Spector \& Jex, 1998), this approach examines a particular behavioral instance, anchoring all subsequent questions to this event (e.g., Diaz \& McMillan, 1991; Hahn, 2000). Participants provided additional information regarding this particular conflict including with whom this conflict occurred (i.e., nurse manager, physician, nurse peer, patient/family) and on what day of the week it occurred (e.g., Monday, Friday; see Appendix B).

Perceived control. A single item was used to assess perceived control (see Appendix B). Specifically, participants rated how controllable (on their part) the weekly 
conflict they identified was to them on a 5-point Likert-type scale ranging from 0 (not at all) to 4 (very; Stone \& Neale, 1984). Although single item measures have an increased likelihood of unreliability, single item measures of perceived control are commonly used throughout the coping literature (e.g., Carver et al., 1989; Kendall \& Terry, 2008; Stone \& Neale, 1984; Terry \& Hines, 1993), particularly in repeated measures assessments (e.g., Park et al., 2004; Zakowski et al., 2001). Moreover, it has been suggested that criticisms of single-item indicators, in general, may be overestimated (Wanous \& Hudy, 2001). Indeed, Wanous, Reichers and Hudy (1997) point out that for self-reported factual data (e.g., number of previous jobs, occupational tenure) and psychological constructs that are not complex (e.g., satisfaction, control) single item measures are often psychometrically sufficient. Therefore, since single item measures of perceived control are common in the coping literature and are often considered psychometrically sufficient this is an appropriate approach.

Weekly coping frequency. To assess coping frequency, participants completed an adapted version of the Brief COPE (Carver, 1997), where they responded to the question "How much did you use this response in connection with your stressful negative conflict?" (see Appendix B). The Brief COPE is a shorter version of the original COPE (Carver et al., 1989), containing 28 items with two items per each of the 14 subscales. The COPE was created according to theoretical rationale, as opposed to empirical data, to comprehensively and meaningfully represent how people cope with stressors. Consistent with the coping literature (e.g., Stone \& Neale, 1984) and the procedure established by Park et al. (2004; see An Appropriate Approach section in Chapter 4), I selected one 
representative item from each of eight subscales of the Brief COPE which loaded highest on the higher order factors of active coping, positive reinterpretation/growth, humor, religion, emotional social support, mental disengagement, behavioral disengagement, and instrumental social support in the original article on the COPE (Carver et al., 1989). Example items include "I took action to try to make the situation better" (active coping), "I turned to work or other activities to take my mind off things" (mental disengagement), and "I got help and advice from other people" (instrumental social support). Although participants responded to a ninth item regarding substance use, I did not examine this for effectiveness and did not include it in any analyses (see Coping section in Chapter 3). In response to each item, participants rated their frequency of use of the coping strategy on a 5-point Likert-type scale, ranging from 0 (not at all) to 4 (a lot).

In order to test my hypotheses, I conducted a Confirmatory Factor Analysis (CFA) on the higher order factors of problem-focused and emotion-focused coping. I used data from the baseline survey assessment of the larger ONRP project to conduct my CFA. Specifically, the baseline survey was completed by 406 nurses who provided a onetime response to all of the coping items in the weekly survey used for the current study (see Figure 8), which provides a valid assessment of the coping structure. Consistent with Lazarus and Folkman's (1984) differentiation, active coping (actively doing something about the stressor) represents behavioral efforts, and positive reinterpretation and growth (changing one's cognitive appraisal of the stressor to be more positive or helpful to the individual) represents cognitive efforts in problem-focused coping. The remaining scales 
should represent emotion-focused coping. Specifically, humor coping (laughing about the situation), religious coping (finding comfort in one's religious beliefs), emotional social support (emotional support from others), mental disengagement (turning to other activities to take mind off things), behavioral disengagement (giving up dealing with it), and instrumental social support (getting help and advice from other people) were loaded on the emotion-focused coping latent factor.

Although this uneven number of items between the two factors ( 2 for problemfocused and 6 for emotion-focused) creates potential concern that results may not be representative, this approach is common in the literature as the number of problemfocused and emotion-focused coping strategies are often unbalanced, primarily because there are numerous ways to regulate emotional responses (e.g., Folkman \& Lazarus, 1980; Folkman \& Lazarus, 1985; Park et al., 2004). Also, within the CFA, I allowed the latent factors (e.g., problem-focused coping, emotion-focused coping) to be correlated in the model, as these coping factors are likely related. Indeed, intercorrelations between factors can account for measures that cross factors in the model, thus improving parameter estimation (Bollen \& Lennox, 1991; Maruyama, 1998). Finally, as one item, behavioral disengagement, originally loaded negatively on the emotion-focused coping latent factor, I reverse coded it to be consistent with the other coping items. This is acceptable considering that increases in behavioral disengagement coping (I gave up trying to deal with it) is really withdrawal or the reduction of behavior, which is contrary to increases in the other coping items. In other words, increases in behavioral 
disengagement constitute reductions in coping behavior, whereas increases in all other coping items represent increases in coping behavior.

Model fit indices suggested the 2-factor model with all items retained was a poor fit for the data (see Table 1). Since there are numerous model fit indices within the literature (cf. Hu \& Bentler, 1999), I selected a variety of fit indices that were absolute fit, relative fit, and noncentrality-based. First, the Chi-square test, the most conventional absolute fit index, was significant $\left(\chi^{2}=87.45, p<.001\right)$, suggesting poor model fit. However, the Chi-square test is not the best indicator of fit in practice as it is strongly impacted by sample size, with a bias against large samples. Another absolute fit index, Hoelter's CN, also suggested the model fit was poor $(\mathrm{G}=178)$, as $\mathrm{G}=200$ is the usual cut-off (Bollen \& Liang, 1988). Second, the relative fit index of NFI was .74 and the IFI were slightly better at .78 , but the cutoff for these indices is at least .90 (Hu \& Bentler, 1999), suggesting that this 2 -factor model was not a good fit for the data. The RMSEA, a popular noncentrality-based index, was .09 , suggesting marginal fit, since values at or below .06 are considered a good fit and values above .10 are a poor fit (Hu \& Bentler, 1999).

As this model did not fit the data well, I conducted other CFA's to test for an acceptable fit (Noar, 2003; see Table 1 for model fit comparison data). I began by examining three different 2-factor models where I removed items that had low factor loadings in the original model. First, I removed the lowest loading item (.11), religious coping, and tested this model. Chi-square change was nonsignificant $\left(\chi^{2}=2.45, p>.05\right)$ and most model fit indices only modestly improved, indicating this nested model did not 
fit well and was not better than the original model. Second, I tested another nested model with only humor coping removed, the next lowest loading item (.20). Chi-square change was significant $\left(\chi^{2}=7.22, p<.01\right)$, suggesting this model was a significant improvement over the original 2-factor model. Moreover, model fit indices improved immensely so that the model had an adequate fit with the data. Third, I tested the 2-factor model with both religious coping and humor coping omitted from the model. Although Chi-square change was significant, it was only at the .05 level $\left(\chi^{2}=5.85, p<.05\right)$ and most model fit indices only slightly improved, suggesting that this model did not fit better than the model with only humor coping removed. The poor fit of humor coping with the other coping items may be due to the fact that the original COPE measure did not have humor coping as one of the subscales (Carver et al., 1989). Rather, humor coping was introduced as a subscale in the Brief COPE and loaded on its own unique factor (Carver, 1997), supporting the omission of humor coping from the emotion-focused coping factor in the current model.

As a 3-factor coping structure could potentially have a good model fit (Park et al., 2004), I tested a 3-factor CFA with humor coping removed (Maruyama, 1998; see Table 1). I loaded mental disengagement and behavioral disengagement on a third factor representing avoidance coping. Although the model fit indices slightly improved, Chisquare change from the original 2-factor model was nonsignificant $\left(\chi^{2}=3.55, p>.05\right)$. Therefore, since the 3-factor model was not a significant improvement, I concluded that the 2-factor model with humor coping omitted was the best and most appropriate model for the current study. This is supported by Lazarus and Folkman's (1984) original 2- 
factor coping structure and the argument for the most parsimonious model, as these are often desirable over more complex models (cf. Mulaik et al., 1989).

Therefore, of these models tested, the 2-factor model with humor coping removed was the best model for a number of reasons. This is particularly important as there is not a clear standard for what qualifies as good fit; rather, the goal should be to satisfy a number of fit index criteria to establish good model fit (Kline, 1998). First, the significant Chi-square change from the original 2-factor model suggests a significant improvement of model fit. The other absolute fit index of Hoelter's CN was greater than 200 and AIC decreased by nearly a third, supporting substantial improved fit over the original model. Second, among the relative fit indices with a cutoff at .90, this model satisfied the IFI, but fell just short for the NFI. Third, noncentrality-based indices also provided support for a good fit of the model. Specifically, the RMSEA was acceptable at .06 and the CFI was also acceptable at .90 . Finally, all factor loadings, with the exception of religious coping (.11), ranged from .17 to .78 . The latent factors of problem-focused and emotion-focused coping were correlated at .64, suggesting a substantial relationship between both forms of coping, which is consistent with the general coping literature (e.g., Park et al., 2004; Tennen et al., 2000).

Related to this model, there is evidence suggesting that social support coping has elements of both problem- and emotion-focused coping (e.g., Carver et al., 1989; Cutrona \& Russell, 1990; Folkman \& Lazarus, 1985), making it debatable whether emotional and instrumental social support should both be considered emotion-focused coping. However, in a recent study examining how nurses cope, Welbourne et al. (2007) found that both 
emotional and instrumental social support items from the Brief COPE strongly loaded on the same factor, a support-seeking factor. Furthermore, in a multilevel factor analysis of Brief COPE data, Roesch et al. (2010) found that both social support items loaded on the same social support factor for between- and within-person analyses, further suggesting that separation of these items is not warranted. Therefore, as emotional social support is clearly emotion-focused coping and the instrumental social support item in this study ("I got help and advice from other people") is ambiguous as to whether the effort is directed towards the problem itself (problem-focused coping) or emotional regulation (emotion-focused coping), these should be modeled as emotion-focused coping. However, as this is debatable, I examined correlations and reliabilities between these items and other emotion-focused items to verify this is an appropriate approach (see Table 2). As expected, the strongest correlation between any of the coping frequency items was between these two social support items $(r=.62)$ further supporting their mutual loading on the same factor. Correlations with other emotion-focused coping strategies ranged from .14 to .23 , which, although not strong, were often greater than correlations with problem-focused coping items, supporting their mutual association as emotion-focused coping strategies.

Finally, prior studies have affirmed the Brief COPE as a reliable measure, with alpha coefficients ranging from .64 to .90 across all eight subscales (Carver, 1997). Furthermore, Park et al. (2004) report improved alpha coefficients when internal consistency of the Brief COPE items were examined under the higher order coping subscales of emotion-focused and problem-focused coping. Using three representative 
time points across their daily study for the analysis, Park et al. (2004) produced estimates suggesting good internal consistency of the Brief COPE items, ranging from .85 to .93 for emotion-focused coping and .82 to .85 for problem-focused coping. Internal consistencies in the current study were estimated across three weeks, each representing a week in the beginning, middle and end of the study period, respectively (weeks 3, 8, and 11). Alpha estimates ranged from .50 to .66 for problem-focused coping and .59 to .64 for emotion-focused coping. Although at the low end of acceptability, these alphas were acceptable when considered in the context of typical coping internal consistency estimates, which are typically derived from more coping items than my measure and are often still low (Carver et al., 1993; Carver et al., 1989; Folkman \& Lazarus, 1985). As Carver and colleagues argue, these low alphas should not be grounds for dismissing the items or measure, as low estimates may arise since many people do not use each coping strategy equally, though theoretically related. For example, an individual may employ active coping very often, but only occasionally use positive reframing, which would produce a low estimate for problem-focused coping. Therefore, these alpha coefficients are likely more indicative of disproportionate use of coping strategies and not necessarily poor internal consistency of the measure.

Weekly perceived coping effectiveness. Using the same eight items of the Brief COPE, participants were also asked "How effective was this response in managing the stress from your negative conflict?" (see Appendix B). Participants then provided ratings of their perceived coping effectiveness in dealing with the weekly WIC for each of the eight coping frequency items on a 3-point Likert-type scale, ranging from 0 (not effective 
at all) to 2 (very effective). In the event that a particular coping strategy was not used (i.e., not at all was selected under coping frequency), participants could select a "not applicable" option. As such, each of the eight coping strategies, if used by the participant, had a unique perceived coping effectiveness rating.

As another check to verify the appropriateness of the problem-focused/emotionfocused coping classification of the coping items as determined by the coping frequency CFA, I examined the reliabilities of the corresponding seven weekly coping effectiveness ratings (humor coping omitted) across the same three weeks (weeks 3, 8, and 11) to see if they mirrored the CFA results. Alpha coefficients were very similar and, in several cases, better than the reliability of the coping frequency items as problem-focused coping ranged from .58 to .76 and emotion-focused coping ranged from .59 to .71 . As such, this provided further support to the 2-factor model obtained earlier and I proceeded to use the seven coping effectiveness items retained after omitting humor coping.

Finally, since each coping frequency item was yoked to a specific coping effectiveness item, responses to coping effectiveness were contingent upon whether or not the coping strategy was used that week. This poses a considerable challenge for analysis of coping effectiveness as an outcome. Therefore, I created one perceived coping effectiveness outcome variable by averaging all seven of the coping effectiveness scores per week for each participant. Having one effectiveness outcome is most consistent with my hypotheses, improves my ability to test the goodness of fit hypothesis given the systematic missing data (i.e., if no coping frequency response, there will be no coping effectiveness response), and makes the results of these analyses more meaningful and 
interpretable. For instance, using an average coping effectiveness score across problemfocused and emotion-focused coping is more representative of coping in real-life, as individuals seldom use only one type (e.g., Tennen et al., 2000). In order to verify that the single-item aggregated coping outcome was appropriate, I conducted three CFAs of a single-factor model of coping effectiveness for three weeks of data (weeks 3, 8, and 11). Although all model fit indices were not satisfactory across all three weeks, RMSEA was between .06 and .07 and Hoelters $\mathrm{CN}$ was above 200 for each week, suggesting an acceptable fit. Factor loadings ranged from .24 to .82 across the three weeks. I computed internal consistency estimates of the seven items for each of the three weeks and these were acceptable, ranging from .71 to .74 .

\section{Data Analysis}

Weekly nurse self-report data in the current study were hierarchically structured, with up to twelve interpersonal conflicts and corresponding coping behaviors nested within persons. These nested data permitted the examination of both within- and between-persons differences in relation to the goodness of fit hypothesis. Furthermore, within-person data are frequently unbalanced in nature, as participants often provide an unequal number of observations. In accordance with this, I employed multilevel regression analysis techniques through Hierarchical Linear Modeling (HLM, v7.0; Raudenbush, Bryk, Cheong, \& Congdon, 2010), which allows for estimation of withinand between-person effects and can handle data with missing observations. Below I describe my data preparation, missing data analysis, and the hierarchical regression analyses that I used to examine my hypothesized relationships. 
Data preparation. Consistent with suggestions in the literature (Enders \& Tofighi, 2007), I person-centered each within-person predictor variable (i.e., perceived control, coping frequency). By so doing, I was able to examine the unit change in a person's rating based on their own average rating rather than zero, making interpretations more meaningful. Moreover, person-centering these variables does not affect the regression coefficient value and is interpreted as the effect on the within-person mean of the outcome variable for each unit increase or decrease in the predictor (Enders \& Tofighi, 2007; Kreft, de Leeuw, \& Aiken, 1995). Thus, when perceived control or coping frequency is centered (or equal to 0), the regression coefficient is interpreted as coping effectiveness on weeks with an average level of perceived control or coping frequency for that person.

The between-person predictor, occupational tenure, was grand mean centered. Grand mean centering the predictor variable provides an intercept estimate that represents the expected value of the outcome variable at the mean level of the predictor variable across persons (Hox, 2002). As such, this approach enabled me to assess the average between-person levels of occupational tenure, which also improves the interpretation of such estimates. Centering both the within- and between-person variables reduces the likelihood of multicollinearity and improves the model estimations when examining main effects and interactions within the same model (Bryk \& Raudenbush, 1992).

Finally, my estimation of interaction effects for Hypothesis 1 and Hypothesis 2 required additional data preparation. To create the interaction variables at Level 1 or the within-person level (Hypothesis 1), I multiplied the person-centered coping frequency 
and perceived control variables, so that the main effects coefficients represent the conditional effects of the predictors at the person's average levels of the other (Aiken \& West, 1991). To create the cross-level interaction terms for Hypothesis 2, I multiplied the person-centered values of the Level 1 interaction terms obtained for Hypothesis 1 (Control x Problem-Focused Coping Frequency, Control x Emotion-Focused Coping Frequency) with the grand mean-centered Level 2 (between-persons level) variable (occupational tenure) to obtain the moderating variables. This approach is consistent with the procedure recommended by Davison, Kwak, Seo and Choi (2002) and Preacher, Curran, and Bauer (2006) when examining cross-level interactions.

Missing data. Within any longitudinal dataset, missing data pose a considerable challenge in analysis. It is important to note that there are different types of missing data such as missing at random (MAR), missing completely at random (MCAR), and nonignorable missing (NIM; Little \& Rubin, 1987). NIM poses some unique challenges for estimations as they vary according to some other variable (i.e., systematic) and require accurate modeling in order to produce valid conclusions (Allison, 2002). The most typical type of missing data for multilevel models, however, is MAR, as MCAR is a more restrictive assumption and less realistic for this type of data (Hox, 2002). Moreover, some individuals may be more likely to drop out or not complete surveys than others in longitudinal multilevel data, usually producing data consistent with MAR (Hox, 2002). However, as violations of this assumption about missing data can greatly affect analysis outcomes (Hox, 2002) it is important to examine the data for systematic differences in missing data before conducting analyses. 
To address the possible issue that the data were not MAR, I examined the dataset to determine the number of missing data points (i.e., surveys, responses to study variables) in a number of ways. First, I looked for systematic missing data not contingent upon participant characteristics or their participation. Only one instance occurred where, due to a technical problem with the Internet data collection software program, data from Week 1 for the coping frequency variable of instrumental social support (i.e., "I got help and advice from other people") was unable to be downloaded and thus not included in analyses. I also examined the missing data for any signs of early attrition from the study, such as missing data being more prevalent near the end of the study, by calculating the number of surveys missing for each week of the study. There was an average of 30 missing weekly data points per week, ranging from 20 to 36 . These missing weekly data points appeared to be randomly missing as the maximum number of missing data occurred both during the middle of the study (week 5) and at the end (week 12). Moreover, one of the lowest numbers of missing data (25) was near the end of the study (week 9), suggesting early attrition was not a systematic issue.

Second, I investigated the study variables, including participant characteristics that might explain missing data. When examining the number of weeks of data submitted by participants, I found that only 19 participants provided less than half ( $\leq 6$ weeks) of the weekly data, as opposed to the 124 nurses who completed more than half of the weekly data. Further mean difference analyses of participant characteristics produced a significant difference in occupational tenure between those completing less than half of the weekly data $(M=10.1, S D=8.09)$ and those completing more than half $(M=17.4, S D$ 
$=12.04 ; t(140)=-3.33, p<.01)$, suggesting that those with less occupational tenure completed fewer weeks, on average, in the study. No other significant mean differences emerged for the study variables of organizational tenure, problem- or emotion-focused coping frequency, coping effectiveness, age or hours worked per week.

Finally, I examined correlations between study variables and missing data. These analyses revealed two significant associations for missing data. First, age was positively associated with number of weeks completed $(r=.09, p<.01)$. Second, consistent with the mean difference analyses of occupational tenure reported above, number of weeks completed was positively related to occupational tenure $(r=.16, p<.01)$, suggesting that as tenure increased, so did the number of completed surveys. No other significant relationships were found between missing data and study variables. Although older and more tenured individuals were more likely to complete more surveys, which does pose certain implications for the limitations of this study, I concluded that any missing data in this dataset is likely MAR.

It should be noted that HLM is a robust method of examining this type of unbalanced data, which is a great strength of this analysis strategy. For instance, in addressing concerns for missing data, Raudenbush and Bryk (2002) argue that as long as every participant provides at least one observation, HLM can be used to analyze the data. The HLM v7.0 statistical program makes this possible by weighting cases that include fewer observations less than those who provide more observations (Raudenbush et al., 2010). Therefore, HLM offers a particularly desirable method of analyzing data with missing data points. This is not to say, however, that missing data is not an important 
consideration, particularly in relation to patterns and reasons behind the missing data. Therefore, although using HLM improves confidence in the validity of the results, missing data patterns still pose potential study limitations, which I discuss further in Chapter 8.

Hypotheses testing analyses. Consistent with the approach of Park et al. (2004), I used hierarchical regression modeling to examine my hypotheses derived from the goodness of fit hypothesis. Hierarchical regression specifies a within-person equation (Level 1 model) and a between-person equation (Level 2 model), producing an estimate for both within- and between-person variable parameters. For the Level 1 (within-person level) model, coping effectiveness was modeled as a function of ratings of perceived control and coping efforts (i.e., problem-focused, emotion-focused) for a given week. This can be conceptualized as each person having their own regression equation for goodness of fit. In the Level 2 equation (between-person level), the within-person intercept and slope estimates derived from the Level 1 model were regressed on betweenperson predictors (i.e., occupational tenure). In the case of Hypothesis 1 where no between-person predictor is specified, the average within-person associations can be tested to observe whether they are significantly different from zero. In relation to Hypothesis 2, however, occupational tenure (between-person predictor) was modeled as a predictor of the within-person estimates from Level 1. Finally, as Hypothesis 3 contains only a between-person predictor, the average between-person associations, similar to Hypothesis 1, can be tested for significance. Hence, using this statistical approach 
allowed me to simultaneously measure the effects of within-and between-person predictors of coping effectiveness and frequency across all my hypotheses.

Furthermore, these models also permitted an examination of the variance components of the within-person parameter estimates of Level 1. Indeed, to account for the possibility of variation across individuals on the Level 1 variables (including the Level 1 interaction terms), I modeled each intercept and slope as random effects, allowing them to vary across persons and to be tested as to whether the slopes vary significantly across persons. Specifically, by allowing the slopes to vary (i.e., setting them as random), the slopes can be tested directly as to whether the within-person matching of coping with control appraisals, as specified in Hypothesis 1, vary significantly across individuals. This is consistent with Park et al.'s (2004) approach and accounts for the potential that goodness of fit (represented by the interaction terms) might work better for some individuals rather than others. Therefore, I allowed the interaction slopes to vary so I could examine Hypothesis 1 as a function of each single person, rather than restricting the analysis to averages across the entire sample. Lastly, in accordance with Snijders and Bosker's (1999) recommendations, I fixed all the cross-level interaction slopes for Hypothesis 2, not allowing them to vary. This improves model estimation and is the most appropriate approach since there is no theoretical rationale to expect these slopes to vary between persons.

Finally, this type of longitudinal data can also be affected by serial dependency. That is, data points that are closest in temporal proximity can be more similar than those that are further apart (Judd \& Kenny, 1981). Serial dependencies can be a significant 
issue when examining temporally ordered data (e.g., West \& Hepworth, 1991), threatening the internal validity of the study. Although the literature has not clearly established a trend pattern for WIC or coping with WIC, it is possible that participants may have had some reactivity to the surveys, giving rise to weekly trends or serial dependency. Consequently, I examined the data for these potential threats to internal validity by making weekly contrasts. Specifically, I created eleven dummy variables as weekly orthogonal contrasts modeled as fixed effects (see Bryk \& Raudenbush, 1992, p. 151) and I used Week 11 as the reference week. No significant relationships resulted between any of the weeks and the coping effectiveness outcome model (Hypothesis 1 and 2). However, in relation to Hypothesis 3a, Week 1 and Week 2 were both positively predictive of problem-focused coping frequency $(b=.39$, .46, respectively; $p<.01)$. Moreover, Week 1 was positively associated with emotion-focused coping frequency (Hypothesis $3 \mathrm{~b} ; b=.21, p<.05$ ). This suggests that coping frequencies, on average, were higher during the initial two weeks of the study relative to other weeks (see Limitations section in Chapter 8 for further discussion of this issue). As there were significant effects, I retained the weekly contrasts in all models examining coping frequency as an outcome. This approach permitted me to account for any variance attributed to temporal sequencing as well as rule out alternative explanations.

Research question analysis. In order to explore the relationship between occupational tenure and coping frequency variation, I computed one standard deviation across all eight coping items for each individual. These standard deviations represented the amount of variance in coping frequency per person (person coping variation) or, in 
other words, they represented the coping mechanisms (coping repertoire) that the individual uses to cope with WIC. Thus, larger standard deviations represent a greater fluctuation between frequency of using the coping strategies (smaller coping repertoire) and smaller standard deviations represent a lesser fluctuation between frequencies of the coping strategies used (larger coping repertoire). I then conducted ordinary least-squares linear regression where these standard deviations were regressed on occupational tenure, offering an investigatory approach of the relationship between occupational tenure and one's coping repertoire. As such, results from this analysis were used to explore the relationship between occupational tenure and one's coping repertoire. 


\section{Chapter 7: Results}

\section{Descriptive Information}

Across the 1,716 total weeks in the study (143 participants x 12 weeks), a total of 1,057 conflicts were reported with a mean of 7.50 conflicts per participant $(S D=3.23)$. Sixty-seven percent of participants provided more than 6 conflicts over the 12-week study period with a maximum of twelve conflicts and a minimum of one conflict given by any one participant. An average of 88 conflicts occurred each week $(S D=8.46)$ among all participants. Compliance (total number of surveys submitted electronically) for the 143 nurse participants over the course of the 12-week study was comparable to other longitudinal studies (e.g., Mohr et al., 2005; Park et al., 2004) with 1,356 out of 1,716 (79\%) possible surveys completed. Participants demonstrated an average completion rate

of 9.5 weeks over the 12 -week study period $(S D=2.67)$. The most commonly reported conflicts were those with nurse peers $(37.2 \%)$ and with patients/others $(29.2 \%)$ while conflicts with nurse managers $(15.4 \%)$ and physicians $(14 \%)$ were reported less often. These findings are contrary to many studies identifying physician aggression as the most prevalent (e.g., Farrell, 1999). Half of all the reported weekly conflicts were also indicated as the most negative stressful event at work for that week.

Means and standard deviations between the specific coping frequency and effectiveness items are reported in Table 2. Across all conflicts, mean perceived control was low, active coping was reported as the most frequently used coping strategy, and the highest effectiveness ratings, on average, were for seeking emotional social support and religious coping. Perceived control was significantly positively related to most of the 
individual coping effectiveness ratings including active coping, positive reframing, emotional social support, and mental disengagement ( $r$ values ranged from .11 to .18, all $p$ values $<.01)$. This suggests that nurses with higher perceived control, on average, reported greater effectiveness for most coping strategies. Interestingly, nearly every coping frequency rating was strongly and positively associated with the corresponding coping effectiveness rating ( $r$ values ranged from .40 to .69 , all $p$ values $<.01$ ) with behavioral disengagement showing a weaker, but positive, correlation $(r=.21, p<.01)$. This suggests that, on average, nurses who used more of a particular coping strategy reported greater coping effectiveness of that strategy.

Additional descriptive results involving the higher-order study variables (i.e., problem-focused coping, emotion-focused coping, coping effectiveness) are displayed in Table 3. Of interest, perceived control was positively associated with problem-focused coping, emotion-focused coping and coping effectiveness, meaning that nurses who had greater perceived control also reported increased use of problem-focused coping and emotion-focused coping, as well as greater coping effectiveness. In relation to coping effectiveness, problem-focused and emotion-focused coping were both significantly positively related while hours worked per week was negatively associated with coping effectiveness and weekly coping variation. Thus, nurses who used more problem-focused coping and emotion-focused coping reported greater coping effectiveness, whereas those who worked more hours per week had, on average, lower coping effectiveness and used more coping strategies. Finally, occupational tenure was significantly positively related to 
emotion-focused coping frequency, suggesting that increases in nursing tenure produce more frequent use of emotion-focused coping, though this relationship was not strong.

\section{Hypothesis Tests}

Hypothesis 1. For Hypothesis 1a, I predicted problem-focused coping to be more effective on weeks when control was higher versus weeks when control was lower. In Hypothesis $1 \mathrm{~b}$, I predicted emotion-focused coping to be more effective on weeks when control was lower compared to weeks when control was higher. These predictions are both consistent with the goodness of fit hypothesis (Lazarus \& Folkman, 1984). To test whether Hypothesis 1a and Hypothesis 1b were supported by the data, I used the following model, paying particular attention to the interaction terms:

$$
\begin{aligned}
\text { Coping Effectiveness } & \mathrm{Ej}_{\mathrm{ij}}=\mathrm{b}_{0 \mathrm{j}}+\mathrm{b}_{1 \mathrm{j}}\left(\mathrm{PF}_{\mathrm{ij}}\right)+\mathrm{b}_{2 \mathrm{j}}\left(\mathrm{EF}_{\mathrm{ij}}\right)+\mathrm{b}_{3 \mathrm{j}}\left(\text { Control }_{\mathrm{ij}}\right)+\mathrm{b}_{4 \mathrm{j}}\left(\mathrm{PF}_{\mathrm{ij}} \mathrm{x}\right. \\
\text { Control } \left._{\mathrm{ij}}\right) & +\mathrm{b}_{5 \mathrm{j}}\left(\mathrm{EF}_{\mathrm{ij}} \mathrm{x} \operatorname{Control}_{\mathrm{ij}}\right)+\mathrm{e}_{\mathrm{ij}} \\
\mathrm{b}_{0 \mathrm{i}} & =\gamma_{00}+\mathrm{u}_{0 \mathrm{i}} \\
\mathrm{b}_{1 \mathrm{i}} & =\gamma_{10}+\mathrm{u}_{1 \mathrm{i}} \\
\mathrm{b}_{2 \mathrm{i}} & =\gamma_{20}+\mathrm{u}_{2 \mathrm{i}} \\
\mathrm{b}_{3 \mathrm{i}} & =\gamma_{30}+\mathrm{u}_{03 \mathrm{i}} \\
\mathrm{b}_{4 \mathrm{i}} & =\gamma_{40}+\mathrm{u}_{4 \mathrm{i}} \\
\mathrm{b}_{5 \mathrm{i}} & =\gamma_{50}+\mathrm{u}_{5 \mathrm{i}}
\end{aligned}
$$

As a first step, I began testing this complex model by examining the unconditional model at Level 1, where no predictors are included. This permits the calculation of the intraclass correlation coefficient (ICC) to determine the variance attributable to between-persons. The ICC(1) is used as a descriptive index of the relative proportion of variance in an 
outcome variable attributable to between- and within-person factors at Level 1. Therefore, as ICC(1) was .30 , this can be interpreted such that $30 \%$ of the variation in coping effectiveness was attributable to between-persons and $70 \%$ to within-persons. Next, I examined the main effects of each of the main predictors (i.e., perceived control, problem-focused coping, emotion-focused coping) in the model without the interaction terms due to the difficulty in interpreting main effects once interaction terms are introduced into the equation chiefly due to multicollinearity issues (Snijders \& Bosker, 1999). Each of the Level 1 (within-person level) variables were person-centered and slopes were permitted to vary. As expected, both coping variables positively predicted coping effectiveness while perceived control had a similar positive effect on coping effectiveness (see Main Effects Model results in Table 4). These findings are consistent with the assumptions behind the goodness of fit hypothesis, where control and coping are assumed to positively predict coping effectiveness (Lazarus \& Folkman, 1984).

Slopes (between-persons variance components) varied significantly only for problem-focused coping, meaning that individuals demonstrated significant variation in the strength of the relationship between problem-focused coping and coping effectiveness. In order to understand and interpret the between-persons variance component values in each table (Tables 4-8, and 10), I report the standard deviations, that is, the square root of the variance component values. As such, these values can be used to compute the range where most individuals lie $( \pm 2 S D)$ in relation to the fixed effect, or average across the individuals. Using problem-focused coping as an example, on average, individuals displayed a positive effect from problem-focused coping on coping 
effectiveness (i.e., unstandardized coefficient of $b=.06$ ), which is significantly different from zero. However, this effect varied across persons, with most people being within 2 standard deviations of this mean, or a range of -.11 to .23 . Therefore, although the average effect of problem-focused coping on coping effectiveness across the sample was positive, some individuals demonstrated a negative relationship. However, the same is not true for emotion-focused coping or perceived control. Therefore, on average, all individuals demonstrated a significant positive effect of emotion-focused coping and perceived control on coping effectiveness, with no significant variation between-persons in this relationship.

I then proceeded to the full analysis of Hypothesis 1 by introducing the interaction terms into the model. Specifically, the Control x Problem-Focused Coping and Control $\mathrm{x}$ Emotion-Focused Coping interaction terms provide variables in which Hypothesis 1a and Hypothesis $1 b$, respectively, can be tested. The interaction terms were person-centered since they were created from the person-centered variables of control and coping frequencies. The slopes of these interaction terms were allowed to vary. Results of this analysis are displayed in Table 4.

Contrary to my predictions, the interaction terms did not significantly predict coping effectiveness, providing no support for Hypothesis 1. Therefore, these results suggest that control does not interact with problem- or emotion-focused coping in predicting coping effectiveness. More specifically, contrary to Hypothesis 1a, under conditions of higher control, problem-focused coping is not more effective compared to conditions of lower control. Similarly, under conditions of lower control, emotion- 
focused coping is not more effective relative to conditions of higher control, which is contrary to Hypothesis 1b. As such, the results of this analysis provide no support for Hypothesis 1, directly contradicting the goodness of fit hypothesis.

As displayed in Table 4, the between-persons variance component of Control $\mathrm{x}$ Emotion-Focused Coping interaction approached significance $(p=.09)$. This suggests that the effect of Control $x$ Emotion-Focused Coping on coping effectiveness may significantly differ between persons or, in other words, that Hypothesis $1 \mathrm{~b}$ could be upheld by some individuals, despite not being supported across the sample on average. As such, I proceeded to test for significant differences between the simple slopes using the procedure established by Preacher, Curran, and Bauer (2006) for a 2-way interaction between Level 1 (within-person level) predictors. Specifically, I plotted three values of control (two $S D$ below mean, the mean, and two $S D$ above the mean) to represent lower, average, and higher levels of control in testing for significant differences between the slopes. This enabled me to detect whether the slopes of individuals across weeks of higher, average, and lower control were significantly different from each other. The results from this test were nonsignificant $(p>.05)$, suggesting that the interaction between emotion-focused coping and control in predicting coping effectiveness does not differ significantly under conditions of lower, average, or higher control. It is possible, however, since the slopes between persons were marginally significantly different, that certain individuals may exhibit greater coping effectiveness using emotion-focused coping under conditions of lower control. However, this is not a strong or significant effect and is unlikely, providing very little to no support for Hypothesis $1 b$. 
Hypothesis 2. In Hypothesis 2, I predicted that occupational tenure would have a significant moderating effect on Hypothesis $1 \mathrm{a}$ and $1 \mathrm{~b}$, or the goodness of fit hypothesized relationships. Specifically, for Hypothesis 2a, I expected the problemfocused coping-high control relationship (Hypothesis 1a) to be stronger for those with greater occupational tenure compared to those with less occupational tenure. Similarly, under Hypothesis 2b, I predicted that the emotion-focused coping-low control relationship (Hypothesis 1b) would be stronger for those with greater occupational tenure relative to those with less occupational tenure. To test Hypothesis 2, I added Level 2 (between-persons) equations to the model from Hypothesis 1:

$$
\begin{aligned}
& \text { Coping Effectiveness } \text { Ej }_{\mathrm{ij}}=\mathrm{b}_{0 \mathrm{j}}+\mathrm{b}_{1 \mathrm{j}}\left(\mathrm{PF}_{\mathrm{ij}}\right)+\mathrm{b}_{2 \mathrm{j}}\left(\mathrm{EF}_{\mathrm{ij}}\right)+\mathrm{b}_{3 \mathrm{j}}\left(\operatorname{Control}_{\mathrm{ij}}\right)+\mathrm{b}_{4 \mathrm{j}}\left(\mathrm{PF}_{\mathrm{ij}} \mathrm{x}\right. \\
&\text { Control } \left._{\mathrm{ij}}\right)+\mathrm{b}_{5 \mathrm{j}}\left(\mathrm{EF}_{\mathrm{ij}} \mathrm{x} \text { Control }_{\mathrm{ij}}\right)+\mathrm{e}_{\mathrm{ij}} \\
& \mathrm{b}_{0 \mathrm{j}}=\gamma_{00}+\gamma_{01}\left(\text { OccTenure }_{\mathrm{ij}}\right)+\mathrm{u}_{0 \mathrm{i}} \\
& \mathrm{b}_{1 \mathrm{i}}=\gamma_{10}+\mathrm{u}_{1 \mathrm{i}} \\
& \mathrm{b}_{2 \mathrm{i}}=\gamma_{20}+\mathrm{u}_{2 \mathrm{i}} \\
& \mathrm{b}_{3 \mathrm{i}}=\gamma_{30}+\mathrm{u}_{03 \mathrm{i}} \\
&\left.\mathrm{b}_{4 \mathrm{j}}=\gamma_{40}+\gamma_{41} \text { OCcTenure }_{\mathrm{ij}}\right)+\mathrm{u}_{4 \mathrm{i}} \\
&\left.\mathrm{b}_{5 \mathrm{j}}=\gamma_{50}+\gamma_{51} \text { OccTenure }_{\mathrm{ij}}\right)+\mathrm{u}_{5 \mathrm{i}}
\end{aligned}
$$

Of particular interest in these equations are the $\gamma_{41}$ and $\gamma_{51}$ coefficients, where the crosslevel interactions between occupational tenure (Level 2) and the control $\mathrm{x}$ coping interaction terms (Level 1) are represented. As the model I used for Hypothesis 2 is an extension of the model for Hypothesis 1, ICC(1) remains the same (.30) and the estimated effects of the control, coping, and control x coping interaction terms have already been 
calculated. Therefore, the main effect of occupational tenure was the only main effect remaining to be estimated. To begin my testing of Hypothesis 2, I grand mean centered occupational tenure and entered it as a fixed Level 2 predictor of coping effectiveness with no other predictors in the model. This was nonsignificant $(p>.05)$.

Next, I entered occupational tenure as a Level 2 (between-persons level) fixed predictor of the intercept (first Level 2 equation shown above) for coping effectiveness to test for a main effect in the larger model where the Level 1 (within-person level) variables were included (i.e., control, coping frequency, control x coping interaction terms). Occupational tenure continued to be a nonsignificant predictor $(p>.05)$. Finally, I tested the full combined hypothesized model, where I entered occupational tenure as a Level 2 fixed predictor of the slopes of the Level 1 (within-person level) interaction terms (latter two models shown above or $b_{4 j}$ and $b_{5 j}$ ). This created the Occupational Tenure $x$ Control $\mathrm{x}$ Coping cross-level interaction terms $\left(\gamma_{41}\right.$ and $\left.\gamma_{51}\right)$, permitting a test of Hypothesis 2. Similar to the test of Hypothesis 1, the within-person variables of control, coping, and interactions terms were person-centered and slopes were permitted to vary.

Contrary to Hypothesis $2 \mathrm{a}$ and Hypothesis $2 \mathrm{~b}$, both cross-level interaction terms were nonsignificant $(p>.05$; see Table 5). Therefore, these results suggest that occupational tenure does not demonstrate a moderating effect on the relationship between coping and control in predicting coping effectiveness. In other words, contrary to Hypothesis 2a, those with greater occupational tenure do not exhibit stronger problemfocused coping $\mathrm{x}$ control relationships with coping effectiveness relative to their lower occupational tenure counterparts. Moreover, a similar conclusion can be drawn regarding 
Hypothesis $2 b$, as those with greater occupational tenure do not tend to have stronger emotion-focused coping $\mathrm{x}$ control associations with coping effectiveness relative to those with less occupational tenure. Therefore, the results of this analysis provide no support for Hypothesis 2.

Hypothesis 3. In Hypothesis 3, I predicted a significant relationship between occupational tenure and coping frequency. Specifically, Hypothesis 3a predicted that those with greater occupational tenure would engage in more problem-focused coping than those with less tenure. Hypothesis $3 \mathrm{~b}$, on the other hand, specified that those with less occupational tenure would utilize more emotion-focused coping than those with more tenure. In order to test Hypothesis 3a and Hypothesis 3b, I used the following models:

$$
\begin{aligned}
& \text { PF Coping Frequency } y_{\mathrm{ij}}=\mathrm{b}_{0 \mathrm{i}}+\mathrm{b}_{1 \mathrm{i}}(\text { Week } 1)+\mathrm{b}_{2 \mathrm{i}}(\text { Week } 2)+\mathrm{b}_{3 \mathrm{i}}(\text { Week } 3)+ \\
& \mathrm{b}_{4 \mathrm{i}}(\text { Week } 4)+\mathrm{b}_{5 \mathrm{i}}(\text { Week } 5)+\mathrm{b}_{6 \mathrm{i}}(\text { Week } 6)+\mathrm{b}_{7 \mathrm{i}}(\text { Week } 7)+\mathrm{b}_{8 \mathrm{i}}(\text { Week } 8)+ \\
& \mathrm{b}_{9 \mathrm{i}}(\text { Week } 9)+\mathrm{b}_{10 \mathrm{i}} \quad\left(\text { Week 10) }+\mathrm{b}_{11 \mathrm{i}}(\text { Week } 12)+\mathrm{e}_{\mathrm{it}}\right. \\
& \mathrm{b}_{0 \mathrm{i}}=\gamma_{00}+\gamma_{01}\left(\text { OccTenure } \mathrm{ij}_{\mathrm{ij}}\right)+\mathrm{u}_{0 \mathrm{i}} \\
& \mathrm{b}_{1 \mathrm{i}}=\gamma_{10}+\mathrm{u}_{1 \mathrm{i}} \\
& \mathrm{b}_{2 \mathrm{i}}=\gamma_{20}+\mathrm{u}_{2 \mathrm{i}} \\
& \mathrm{b}_{3 \mathrm{i}}=\gamma_{30}+\mathrm{u}_{3 \mathrm{i}} \\
& \mathrm{b}_{4 \mathrm{i}}=\gamma_{40}+\mathrm{u}_{4 \mathrm{i}} \\
& \mathrm{b}_{5 \mathrm{i}}=\gamma_{50}+\mathrm{u}_{5 \mathrm{i}} \\
& \mathrm{b}_{6 \mathrm{i}}=\gamma_{60}+\mathrm{u}_{6 \mathrm{i}} \\
& \mathrm{b}_{7 \mathrm{i}}=\gamma_{70}+\mathrm{u}_{7 \mathrm{i}}
\end{aligned}
$$




$$
\begin{aligned}
& \mathrm{b}_{8 \mathrm{i}}=\gamma_{80}+\mathrm{u}_{8 \mathrm{i}} \\
& \mathrm{b}_{9 \mathrm{i}}=\gamma_{90}+\mathrm{u}_{9 \mathrm{i}} \\
& \mathrm{b}_{10 \mathrm{i}}=\gamma_{100}+\mathrm{u}_{10 \mathrm{i}} \\
& \mathrm{b}_{11 \mathrm{i}}=\gamma_{110}+\mathrm{u}_{11 \mathrm{i}}
\end{aligned}
$$

EF Coping Frequency $y_{i j}=b_{0 i}+b_{1 i}\left(\right.$ Week 1) $+b_{2 i}\left(\right.$ Week 2) $+b_{3 i}($ Week 3) + $b_{4 i}\left(\right.$ Week 4) $+b_{5 i}\left(\right.$ Week 5) $+b_{6 i}\left(\right.$ Week 6) $+b_{7 i}\left(\right.$ Week 7) $+b_{8 i}($ Week 8) + $b_{9 i}($ Week 9$)+b_{10 i} \quad\left(\right.$ Week 10) $+b_{11 i}\left(\right.$ Week 12) $+e_{i t}$

$$
\begin{aligned}
& \left.b_{0 i}=\gamma_{00}+\gamma_{01} \text { OccTenure }_{i j}\right)+u_{0 i} \\
& b_{1 i}=\gamma_{10}+u_{1 i} \\
& b_{2 i}=\gamma_{20}+u_{2 i} \\
& b_{3 i}=\gamma_{30}+u_{3 i} \\
& b_{4 i}=\gamma_{40}+u_{4 i} \\
& b_{5 i}=\gamma_{50}+u_{5 i} \\
& b_{6 i}=\gamma_{60}+u_{6 i} \\
& b_{7 i}=\gamma_{70}+u_{7 i} \\
& b_{8 i}=\gamma_{80}+u_{8 i} \\
& b_{9 i}=\gamma_{90}+u_{9 i} \\
& b_{10 i}=\gamma_{100}+u_{10 i} \\
& b_{11 i}=\gamma_{110}+u_{11 i}
\end{aligned}
$$

These models contain both the Level 1 (within-person level) and Level 2 equations, since occupational tenure is entered in at Level 2 as a predictor of the Level 1 intercept $\left(b_{0 i}\right)$. Moreover, since the weekly contrasts were significantly related to the problem- and 
emotion-focused coping frequency outcomes, I retained them as fixed effects in the model to account for any variance these variables explain.

Similar to my procedure for Hypothesis 1, I began testing this model by estimating the ICC(1) from the unconditional models of coping frequency for Level 1 (one for problem-focused coping and one for emotion-focused coping). The problemfocused ICC(1) was .30 and the emotion-focused ICC(1) was .37, affirming the withinpersons approach (about $70 \%$ and $63 \%$ of the variance is attributable to within-persons, respectively). Next, I grand-mean centered occupational tenure in the Hypothesis 3a model as a fixed effect at Level 2 to predict problem-focused coping frequency. Similarly, I followed the same procedure for occupational tenure in the Hypothesis $3 \mathrm{~b}$ model in predicting emotion-focused coping frequency. No significant relationships emerged between occupational tenure and coping frequency for either problem-focused or emotion-focused coping (see Table 6). Indeed, despite occupational tenure and emotion-focused coping frequency being positively significantly correlated (see Table 3), no significant relationship was identified in the testing of these models. Therefore, both Hypothesis $3 \mathrm{a}$ and $3 \mathrm{~b}$ were not supported by the data.

\section{Research Question Tests}

Research Question 1. In Research Question 1, I predicted a relationship between occupational tenure and person coping variation or, in other words, that greater tenure would predict an individual's variance in coping frequency across the 12 study weeks. In order to examine this question, I used ordinary least squares regression where person coping variation, computed as a single standard deviation score from all eight coping 
frequency scores for each person across all twelve weeks, was regressed on occupational tenure. As person coping variation was not significantly correlated with any study variable (see Table 3), it was not surprising that this regression analysis produced no evidence of a significant relationship (see Table 7). Moreover, this sample had restricted variability in occupational tenure with fewer early career nurses (see Participants section in Chapter 6), which may limit the ability to detect a significant difference using ordinary least squares regression.

In summary, the results from my analyses produced no evidence consistent with my hypothesized relationships. I found no significant effect of the goodness of fit interactions in predicting coping effectiveness (Hypothesis 1), occupational tenure was not a significant moderator of the goodness of fit predictions (Hypothesis 2), and occupational tenure was not a significant predictor of problem- or emotion-focused coping frequency (Hypothesis 3). Moreover, I found no evidence to support a relationship between occupational tenure and person coping variation, contrary to Research Question 1. However, there are a few alternative methods that can be adopted to examine these predicted relationships. For instance, the goodness of fit hypothesis was originally supported by evidence of perceived control positively predicting problem-focused coping and negatively predicting emotion-focused coping, offering another way to examine Hypothesis 1 (Folkman et al., 1986; Folkman \& Lazarus, 1980). Second, the literature suggests that organizational tenure, the length of time one spends in a particular organization, may be another way to meaningfully investigate the relationship between tenure and coping (Lu et al., 2010; Sundberg, 2003; Wright \& Bonett, 1993). Finally, 
having a variable representing coping variation on the weekly level rather than the person level enables an examination of this relationship using multilevel regression in HLM, which is a more powerful test than simple regression (Raudenbush \& Bryk, 2002; Snijders \& Bosker, 1998). Therefore, as these offer alternative methods whereby I could examine my hypotheses, I conducted supplemental analyses and summarize these results below.

\section{Supplemental Analyses}

Hypothesis 1 supplemental analysis. Given the null findings for Hypothesis 1, I further explored the within-persons variable of perceived control in predicting coping frequency, which is another way to examine goodness of fit assumptions. Specifically, the goodness of fit hypothesis assumes that control will be positively related to problemfocused coping and negatively related to emotion-focused coping (Lazarus \& Folkman, 1984). Thus, this is also a way to explore goodness of fit following the null findings for Hypothesis 1. This analysis required a modification to the Hypothesis 1 equation by creating one equation with perceived control as the Level 1 (within-person level) predictor of problem-focused coping frequency and another equation with perceived control as the Level 1 predictor of emotion-focused coping frequency. Specifically, I used these models:

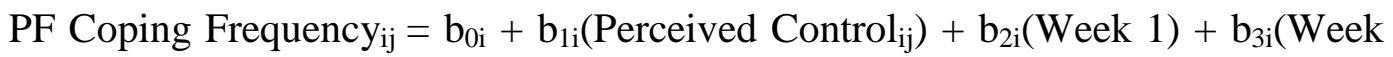

$2)+b_{4 i}\left(\right.$ Week 3) $+b_{5 i}\left(\right.$ Week 4) $+b_{6 i}\left(\right.$ Week 5) $+b_{7 i}\left(\right.$ Week 6) $+b_{8 i}($ Week 7) + $b_{9 i}\left(\right.$ Week 8) $+b_{10 i}\left(\right.$ Week 9) $+b_{11 i}\left(\right.$ Week 10) $+b_{12 i}\left(\right.$ Week 12) $+e_{i t}$

$$
\mathrm{b}_{0 \mathrm{i}}=\gamma_{00}+\mathrm{u}_{0 \mathrm{i}}
$$




$$
\begin{aligned}
& b_{1 i}=\gamma_{10}+u_{1 i} \\
& b_{2 i}=\gamma_{20}+u_{2 i} \\
& b_{3 i}=\gamma_{30}+u_{3 i} \\
& b_{4 i}=\gamma_{40}+u_{4 i} \\
& b_{5 i}=\gamma_{50}+u_{5 i} \\
& b_{6 i}=\gamma_{60}+u_{6 i} \\
& b_{7 i}=\gamma_{70}+u_{7 i} \\
& b_{8 i}=\gamma_{80}+u_{8 i} \\
& b_{9 i}=\gamma_{90}+u_{9 i} \\
& b_{10 i}=\gamma_{100}+u_{10 i} \\
& b_{11 i}=\gamma_{110}+u_{11 i}
\end{aligned}
$$

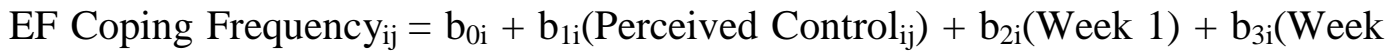
$2)+b_{4 i}\left(\right.$ Week 3) $+b_{5 i}\left(\right.$ Week 4) $+b_{6 i}\left(\right.$ Week 5) $+b_{7 i}\left(\right.$ Week 6) $+b_{8 i}($ Week 7) + $b_{9 i}\left(\right.$ Week 8) $+b_{10 i}\left(\right.$ Week 9) $+b_{11 i}\left(\right.$ Week 10) $+b_{12 i}\left(\right.$ Week 12) $+e_{i t}$

$$
\begin{aligned}
& b_{0 i}=\gamma_{00}+u_{0 i} \\
& b_{1 i}=\gamma_{10}+u_{1 i} \\
& b_{2 i}=\gamma_{20}+u_{2 i} \\
& b_{3 i}=\gamma_{30}+u_{3 i} \\
& b_{4 i}=\gamma_{40}+u_{4 i} \\
& b_{5 i}=\gamma_{50}+u_{5 i} \\
& b_{6 i}=\gamma_{60}+u_{6 i} \\
& b_{7 i}=\gamma_{70}+u_{7 i}
\end{aligned}
$$




$$
\begin{aligned}
& \mathrm{b}_{8 \mathrm{i}}=\gamma_{80}+\mathrm{u}_{8 \mathrm{i}} \\
& \mathrm{b}_{9 \mathrm{i}}=\gamma_{90}+\mathrm{u}_{9 \mathrm{i}} \\
& \mathrm{b}_{10 \mathrm{i}}=\gamma_{100}+\mathrm{u}_{10 \mathrm{i}} \\
& \mathrm{b}_{11 \mathrm{i}}=\gamma_{110}+\mathrm{u}_{11 \mathrm{i}}
\end{aligned}
$$

In these models, I person-centered perceived control and allowed the slopes to vary. Perceived control was significantly positively predictive of problem-focused coping frequency $(b=.149, p<.001)$. This suggests that increased perceptions of control produced increases in problem-focused coping frequency, which is consistent with goodness of fit assumptions. Although perceived control was not significantly related to emotion-focused coping frequency $(b=.033, p>.05)$, perceived control did demonstrate a significant between-persons variance component $(S D=.122, p<.05)$, supporting significant variation in slopes between individuals. Therefore, although it seems there may be a positive relationship between emotion-focused coping and control (this is not significant), significant slope variation suggests this relationship is very different across individuals. As such, it is plausible that some individuals may actually demonstrate a weaker or even negative relationship between perceived control and emotion-focused coping frequency. Therefore, this is partially supportive of the goodness of fit hypothesis.

Research Question 1 supplemental analyses. In order to probe the relationship between coping variation and tenure further, I investigated organizational tenure as a predictor of person coping variation in place of occupational tenure. The literature suggests that organizational tenure is related to coping (Wright \& Bonett, 1993), offering an additional way to examine the effect of tenure on coping. I entered organizational 
tenure as a single predictor of person coping variation in a simple univariate regression model. Although it was not significant, organizational tenure approached significance $b=$ $.003, t(141)=1.76, p=.08$, explaining approximately $2 \%$ of the variance in coping variation $R^{2}=.021, F(1,141)=3.09, p=.08$. This suggests that organizational tenure may be a better predictor of coping variation than occupational tenure, at least on the person level.

Offering an additional way to examine the relationship between tenure and coping variation (Research Question 1), coping variation can be conceptualized on a weekly rather than person level. By doing this, each person can have up to twelve coping variation scores (standard deviations) that represent the coping variation between the eight coping strategies each week. This enables a more meaningful and fine-grained analysis of this relationship, as each weekly standard deviation represents the fluctuation between eight coping strategies rather than the fluctuation between the potential 96 coping frequency scores (12 weeks x 8 coping strategies) at the person level. Moreover, creating a weekly outcome that can be examined using HLM enables a more powerful approach in detecting significant relationships (Raudenbush \& Bryk, 2002; Snijders \& Bosker, 1998). To create the weekly coping variation variable, I computed a standard deviation score across all eight coping frequency variables for each week of data (as opposed to one standard deviation per individual computed for person coping variation). As this represented a new outcome model, I entered the weekly contrasts as fixed effects to examine for any significant effects. One significant effect emerged, with Week 1 
positively predicting weekly coping variation $(p<.05)$, thus I retained the weekly contrasts. Specifically, I used this model:

$$
\begin{aligned}
& \text { Weekly Coping Variation }{ }_{i j}=b_{0 i}+b_{1 i}\left(\text { Week 1) }+b_{2 i}\left(\text { Week 2) }+b_{3 i}(\text { Week 3) }+\right.\right. \\
& \mathrm{b}_{4 \mathrm{i}}(\text { Week } \quad 4)+\mathrm{b}_{5 \mathrm{i}}\left(\text { Week 5) }+\mathrm{b}_{6 \mathrm{i}}\left(\text { Week 6) }+b_{7 \mathrm{i}}\left(\text { Week 7) }+\mathrm{b}_{8 \mathrm{i}}(\text { Week 8) }+\right.\right.\right. \\
& b_{9 i}\left(\text { Week 9) }+b_{10 i} \quad\left(\text { Week 10) }+b_{11 i}\left(\text { Week 12) }+e_{i t}\right.\right.\right. \\
& b_{0 i}=\gamma_{00}+\gamma_{01}\left(\text { OccTenure }_{i j}\right)+u_{0 i} \\
& b_{1 i}=\gamma_{10}+u_{1 i} \\
& \mathrm{~b}_{2 \mathrm{i}}=\gamma_{20}+\mathrm{u}_{2 \mathrm{i}} \\
& b_{3 i}=\gamma_{30}+u_{3 i} \\
& \mathrm{~b}_{4 \mathrm{i}}=\gamma_{40}+\mathrm{u}_{4 \mathrm{i}} \\
& b_{5 i}=\gamma_{50}+u_{5 i} \\
& \mathrm{~b}_{6 \mathrm{i}}=\gamma_{60}+\mathrm{u}_{6 \mathrm{i}} \\
& \mathrm{b}_{7 \mathrm{i}}=\gamma_{70}+\mathrm{u}_{7 \mathrm{i}} \\
& b_{8 i}=\gamma_{80}+u_{8 i} \\
& \mathrm{~b}_{9 \mathrm{i}}=\gamma_{90}+\mathrm{u}_{9 \mathrm{i}} \\
& \mathrm{b}_{10 \mathrm{i}}=\gamma_{100}+\mathrm{u}_{10 \mathrm{i}} \\
& \mathrm{b}_{11 \mathrm{i}}=\gamma_{110}+\mathrm{u}_{11 \mathrm{i}}
\end{aligned}
$$

Next, I computed the ICC(1) from the unconditional model for Level 1, which equaled .21 , meaning that approximately $79 \%$ of the variance was attributable to within-persons. I then entered occupational tenure (Level 2 predictor) in the model, where I grand-mean centered and fixed the slopes for occupational tenure. This yielded a nonsignificant relationship $(p>.05)$. 
I retained the weekly contrasts and replaced occupational tenure with organizational tenure as a Level 2 (between-persons level) predictor, which was grandmean centered with fixed slopes and entered with no other predictors (other than the weekly contrasts) in the model. Organizational tenure was a significant positive predictor of weekly coping variation $(b=.005, p<.01)$. Therefore, as organizational tenure increased, variation in coping frequency increased, meaning that as nurses gained more experience in the organization, they reported using significantly less coping strategies to deal with stressors on a weekly basis. As such, it seems that nurses who are more knowledgeable with their organizations rely on certain coping strategies more than others such that they have a specialized coping style or ability to use certain coping strategies in certain situations.

Finally, as a next step, I examined the relationship between the coping effectiveness outcome and the predictors of person coping variation and weekly coping variation in separate models. In order to do this, I used the following models:

$$
\begin{aligned}
& \text { Coping Effectiveness } \mathrm{ij}_{\mathrm{ij}}=\mathrm{b}_{0 \mathrm{j}}+\mathrm{e}_{\mathrm{ij}} \\
& \qquad \begin{aligned}
\left.\mathrm{b}_{0 \mathrm{j}}=\gamma_{00}+\gamma_{01} \text { Person Coping Variation }_{\mathrm{ij}}\right)+\mathrm{u}_{0 \mathrm{i}} \\
\text { Coping Effectiveness } \mathrm{P}_{\mathrm{ij}}=\mathrm{b}_{0 \mathrm{j}}+\mathrm{b}_{1 \mathrm{j}}\left(\text { Weekly Coping Variation }_{\mathrm{ij}}\right)+\mathrm{e}_{\mathrm{ij}} \\
\mathrm{b}_{0 \mathrm{j}}=\gamma_{00}+\mathrm{u}_{0 \mathrm{i}} \\
\mathrm{b}_{1 \mathrm{i}}=\gamma_{10}+\mathrm{u}_{1 \mathrm{i}}
\end{aligned}
\end{aligned}
$$

First, I entered person coping variation as a grand-mean centered Level 2 (betweenpersons level) predictor with fixed effects and no other variables entered into the model. This was significant $(b=.35, p<.05)$. Next, I examined weekly coping variation as a Level 1 (within-person level) predictor of coping effectiveness, where I person-centered 
the variable, allowed the slopes to vary and introduced no other variables in the model. This produced a positive significant effect $(b=.26, p<.001)$ and the between-persons variance component was also significant $(S D=.21, p<.05)$, such that the effect of weekly coping variation varied significantly between individuals. Therefore, as individuals used more coping strategies equally (coping frequency $S D$ was smaller), coping effectiveness diminished on average. Or, in other words, when fewer coping strategies were used (coping frequency $S D$ was larger), coping effectiveness improved. However, this effect varied significantly between individuals, making it so that a minority (within $2 S D$ below the mean) demonstrated a negative relationship between weekly coping variation and coping effectiveness. 


\section{Chapter 8: Discussion}

The purpose of this dissertation was to examine coping effectively with WIC in the nursing workplace by taking a within-persons approach to test the goodness of fit hypothesis. Despite several studies on the topic (e.g., Compas et al., 1988; Kendall \& Terry, 2008), the literature, as far as I am aware, continues to lack an investigation of the goodness of fit hypothesis within the workplace. Coping effectively with workplace interpersonal conflict (WIC), especially among nurses, is a research area of critical concern (e.g., Almost, 2006), making it an important context in which to test the goodness of fit hypothesis predictions. In addition, very little research has examined potential moderators of the goodness of fit hypothesis, although there is evidence that such person variables may exist (Park et al., 2004) and occupational tenure, in particular, may be one of those variables (Bradley, 2007). Finally, as suggested by the literature (e.g., Park et al., 2001; Park et al., 2004), it is essential to take an appropriate methodological approach when examining the goodness of fit hypothesis by adopting a within-persons perspective of coping with stressors that fluctuate in perceived control such as social stressors (e.g., WIC). Thus, this dissertation provides a rigorous empirical examination of the goodness of fit hypothesis within the nursing workplace while considering occupational tenure as an influential variable in the coping process using a within-persons analysis technique.

In this discussion, I have summarized the results for Hypothesis 1 through 3 and Research Question 1 individually. First, I review the control-problem-focused coping relationship (Hypothesis 1a) and the control-emotion-focused coping relationship 
(Hypothesis 1b), which represent the goodness of fit hypothesis predictions. Second, I examine the effect of occupational tenure on the interaction between control and coping; in other words, the moderating relationship between occupational tenure and the goodness of fit predictions (Hypothesis 2). Third, I discuss the effect of occupational tenure on the frequency of using problem-focused coping (Hypothesis 3a) and emotionfocused coping (Hypothesis 3b). Finally, I examine the relationship between occupational tenure and coping variability, or the fluctuation in using different coping strategies (Research Question 1). After I summarize these study findings, I discuss the strengths and limitations of this study as well as implications for future research and practical consideration.

\section{The Goodness of Fit Hypothesis}

Despite a solid theoretical foundation, this dissertation produced no substantial evidence consistent with the goodness of fit hypothesis. Although this is surprising since I used an appropriate methodological approach, the literature investigating the goodness of fit hypothesis contains several inconsistencies, which may help explain these findings. First, as I discussed earlier in Chapter 4 (see Chapter 4), many studies only support the problem-focused coping-high control condition (e.g., Conway \& Terry, 1992; Masel et al., 1996; Vitaliano et al., 1990) or the emotion-focused coping-low control condition (e.g., Carver et al., 1993; Terry \& Hynes, 1998; Zakowski et al., 2001) in producing

effective outcomes. Other studies have found evidence consistent with both predictions of goodness of fit (e.g., Compas et al., 1988; Park et al., 2001; Park et al., 2004). Still other studies, including the present one, have found no support for either prediction of the 
goodness of fit hypothesis (e.g., Felton \& Revenson, 1984; Kendall \& Terry, 2008; Roberts, 1995).

Taken together, it seems reasonable to conclude that goodness of fit is likely context-dependent, such that under certain conditions goodness of fit is applicable, but not under all conditions. This line of reasoning is consistent with coping specificity, where effectiveness of any given coping effort relies upon characteristics of the context or situation (Benyamini et al., 2008; Lazarus \& Folkman, 1984). For instance, when goodness of fit is examined in relation to coping with physical pain or chronic disease there is often little support (Felton \& Revenson, 1984; Kendall \& Terry, 2008). However, coping with daily life stressors among young adults and adolescents are contexts in which goodness of fit is well-supported (Compas et al., 1988; Forsythe \& Compas, 1987; Park et al., 2004). The literature suggests that goodness of fit should be upheld among female workers resolving interpersonal conflicts (Portello \& Long, 2001) and in the workplace where perceptions of control often vary considerably (Karasek, 1979; Theorell, 2003). However, it is possible that the nursing work context does not offer appropriate conditions for goodness of fit. In relation to the current study, for instance, average perceived control was low and had little variability, which may have inhibited obtaining support for the hypothesized relationships. Indeed, nursing may be a context in which perceived control in interpersonal conflicts is always low, perhaps due to nursing's history of being an oppressed or subjugated discipline in relation to the field of medicine (cf. Roberts, 1983). 
As another potential explanation, goodness of fit may be more applicable for those who are younger or less experienced in nursing, which is consistent with the fact that most studies examining younger groups find support for goodness of fit (e.g., Park et al., 2004). As such, the matching of perceived control with type of coping may be more relevant for younger or more inexperienced individuals and, as this study was biased toward older tenured nurses, this may have contributed to the null findings. For example, younger or inexperienced nurses are often greatly distressed as they become involved in conflicts with older nurses (Baltimore, 2006; Farrell, 2001). Therefore, when these inexperienced nurses successfully match control with coping efforts, this may produce a greater perceived beneficial effect. Likewise, older or more experienced nurses may become accustomed to conflicts with others at work and goodness of fit may not have much of an effect. In sum, even as coping is a highly contextualized phenomenon (DeLongis \& Holtzman, 2005; Tennen et al., 2000; Todd et al., 2004) it is likely that necessary conditions such as control and demographic variability were not met in this study or it is possible that the nursing workplace may not be an appropriate context in which to examine the goodness of fit hypothesis.

The conclusion that the nursing workplace is not appropriate for goodness of fit seems to be doubtful, since the supplemental analysis produced evidence supportive of mechanisms underlying goodness of fit predictions. For example, increases in perceived control predicted increases in problem-focused coping, which is consistent with the depicted relationships in the transactional model and the underlying assumptions behind the goodness of fit hypothesis. In fact, in the initial attempts to test goodness of fit, many 
studies examined the relationship between perceived control and problem- and emotionfocused coping frequency, finding support for these predictions (e.g., Folkman et al., 1986). This finding is also consistent with other studies supporting the essential role secondary appraisals of control have on choice of coping efforts (e.g., Drumheller et al., 1991; Folkman et al., 1986; Florian et al., 1995). That is, levels of perceived control influence choice of coping strategy and, more specifically, that under conditions of higher control, individuals will make more problem-focused coping efforts wherein the individual can alter or eliminate the problem itself, making this approach the logical choice (Lazarus \& Folkman, 1984; Lazarus, 1993). For example, if a nurse felt that he/she had a great amount of control over the conflict situation, it is more likely that he/she would enact problem-solving coping, by addressing the other party in the conflict directly. As such, the significant relationship between perceived control and problemfocused coping frequency are consistent with the transactional model and the underlying assumptions of the goodness of fit hypothesis.

Additionally, although perceptions of control did not significantly predict emotion-focused coping frequency in the supplemental analysis, this relationship varied significantly between individuals. In other words, for some nurses, perceived control negatively predicted emotion-focused coping, but for others it positively predicted emotion-focused coping. This suggests that the transactional model and goodness of fit hypothesis predictions, which specify a negative relationship between perceived control and emotion-focused coping (Lazarus \& Folkman, 1984; Lazarus, 1993), were upheld by at least some nurses. Therefore, although the perceived control and emotion-focused 
coping were not negatively related across the entire sample on average, a subset demonstrated this relationship. As such, these results offer evidence supportive of underlying mechanisms of goodness of fit as nurses often matched perceived control with coping efforts, though not necessarily having a perceived benefit from doing so.

It is also important to recognize that this study found several significant effects of coping and control on coping effectiveness. Indeed, other studies have found support for the main effects of coping and perceived control on coping outcomes without finding support for the goodness of fit hypothesis itself (Kendall \& Terry, 2008). In the current study, I found that, regardless of type of coping used, coping produced beneficial outcomes. Although this seems straightforward and intuitive, there has been a debate within the literature about whether or not coping actually leads to improvement in outcomes (Aldwin \& Revenson, 1987) and about which types of coping produce beneficial effects (Baker \& Berenbaum, 2007; Kohler et al., 2006; Zeidner \& Saklofske, 1996). However, the results from the current study provide an optimistic point of view as both problem- and emotion-focused coping, including the controversial avoidance (or escape) coping strategies (i.e., behavioral disengagement, mental disengagement; Latack, 1986; Tyler \& Cushway, 1992), produced effective outcomes from the perspective of the individual. Therefore, this study suggests that regardless of the type of coping used, engaging in a coping strategy to deal with WIC will likely lead to a more effective coping outcome than if no coping effort is made.

Second, this study identified emotion-focused coping as a slightly stronger means of effectively coping with WIC than problem-focused coping and that the effectiveness of 
problem-focused coping varied considerably across persons. These represent new findings to the literature, as most studies find problem-focused coping to be more effective generally (Tennen et al., 2000; Zeidner \& Saklofske, 1996) and report the effectiveness of emotion-focused coping to vary between persons, not problem-focused coping (Park et al., 2004). The first finding is consistent with Baker and Berenbaum (2007) where emotional expressive writing (emotion-focused coping) was more effective in dealing with interpersonal stressors than problem-solving writing (problem-focused coping). Therefore, in relation to WIC, emotion-focused coping may be more effective because the conflicts being investigated involved interpersonal stressors.

Moreover, the significant variability in problem-focused coping effectiveness may be indicative of some personality characteristic or individual difference that enables certain nurses to use problem-focused coping more effectively than others. Although occupational and organizational tenure were not significant factors in explaining this variability, other individual difference or personality characteristics may have substantial influences. For instance, locus of control (Hahn, 2000), self-efficacy (Aspinwall \& Taylor, 1997), openness to experience (DeLongis \& Holtzman, 2005), neuroticism or optimism (Park et al., 2004) are all personality factors that could affect the coping process and outcomes. Thus, although emotion-focused coping strategies may be slightly more effective, certain individual difference variables may enhance the ability of nurses to cope effectively with WIC using problem-focused coping strategies.

Finally, this study found a significant positive effect of perceived control on coping effectiveness. A large body of literature has acknowledged control as having a 
direct impact on the stress and coping process in the workplace, often mitigating work strain and other negative outcomes when control is greater (e.g., Karasek, 1979; Theorell, 2003). Control may even have a main effect similar to coping since some theorists regard control itself as a coping mechanism (e.g., Latack, 1986). The results from the current study are consistent with the proposition that control has a positive effect on coping effectiveness. Having more control in conflict situations may enable an individual to more effectively resolve the conflict since more options are likely available or utilized (Lee, 2002; Rahim, 1986). The main positive effect of control was consistent across the results, including the trend towards variation between individuals in regards to the control-emotion-focused coping interaction in predicting coping effectiveness. Although this suggests that some individuals may differ substantially from one another with some demonstrating relationships consistent with goodness of fit predictions, the effect of control, on average, was still positive. In other words, contrary to the goodness of fit prediction of emotion-focused coping being more effective under conditions of lower control, coping effectiveness was consistently predicted by elevated levels of control, regardless of coping.

In sum, no evidence was found supportive of the goodness of fit hypothesis in the current study. This may have been due to a variety of contextual factors such as lack of variability in perceived control or demographics or, nursing may be a context in which goodness of fit does not apply. However, the underlying mechanism of matching perceived control with coping efforts was supported by the supplemental analysis, suggesting that goodness of fit is in operation among nurses, but does not produce 
perceived coping benefits. Finally, the results also support a direct positive effect of problem-focused coping, emotion-focused coping, and perceived control on coping effectiveness in dealing with WIC.

\section{Occupational Tenure and Coping}

Contrary to my hypotheses, the results of this dissertation failed to support occupational tenure as a moderator of the goodness of fit hypothesis (Hypothesis 2) or as a predictor of coping frequency (Hypothesis 3). Given the lack of support for the goodness of fit hypothesis (Hypothesis 1), it may not be surprising that no evidence emerged of occupational tenure affecting the goodness of fit hypothesis in the current study. Much the same as these null findings, the current study also failed to produce evidence of occupational tenure predicting problem- or emotion-focused coping frequency. Indeed, occupational tenure emerged as a factor not important in the prediction of coping effectiveness or coping frequency. In other words, the longer a nurse has been in nursing does not influence how or how well he/she coped with WIC under different conditions of control and coping or in general. Therefore, these results suggest that occupational tenure is not an important predictor of coping effectiveness or coping choice.

First, it is possible that the lack of evidence for occupational tenure as a moderator of goodness of fit could be due to certain study factors. For instance, the current study had a sample skewed towards greater occupational tenured nurses. It is possible that greater variability in occupational tenure could facilitate the identification of an effect of occupational tenure on the goodness of fit hypothesis. Indeed, as highlighted 
above, early career nurses may benefit from goodness of fit more than those who have greater tenure, especially in relation to coping with conflict at work. Therefore, the restricted range of occupational tenure in the current sample may have led to these null findings. Moreover, detection of significant cross-level interaction terms requires a substantial amount of power, which may have been insufficient in the current study. For instance, in their detection of optimism and neuroticism as significant moderators of goodness of fit in a daily diary sample, Park et al. (2004) had over 4,000 observations, which is more than double the current study. Therefore, although the current study had many observations, it is possible that more observations could have led to the detection of a significant effect.

However, even more likely, it seems that another type of tenure, organizational tenure, is more important in determining the effectiveness of coping efforts. This is consistent with other studies that have found a relationship between organizational tenure and coping ( $\mathrm{Lu}$ et al. 2010; Sundberg, 2003; Wright \& Bonett, 1993). Although both tenure variables did not have a direct significant effect on coping effectiveness, organizational tenure did indirectly influence coping effectiveness through coping frequency variation such that as organizational tenure increased, nurses used particular coping strategies more, which increased effectiveness. Therefore, this suggests that organizational tenure, or the amount of time a nurse has spent within a particular organization, is more important in determining effectiveness of coping efforts than the length of time one has been a nurse. For example, having experience with the specific organizational structure and climate as well as the particular coworkers and colleagues of 
an organization may empower the nurse with knowledge of ways to cope effectively with particular conflicts. In other words, greater organizational tenure may enable the nurse to match their coping efforts with specific characteristics of the WIC within the context of the organization, which should yield improved outcomes (Benyamini et al., 2008; Lazarus, 1993). Therefore, having knowledge of and experience in the nursing profession are not as important has having knowledge of and experience in the specific organizational content of the nursing workplace. I discuss this further in relation to coping frequency variation below (Research Question 1; see Chapter 8).

Second, despite the literature's suggestions that greater occupational tenure should lead to increases in problem-focused coping and less occupational tenure should produce more emotion-focused coping, these relationships were not supported by the current study. As a potential explanation, prior studies finding evidence of these associations have primarily used cross-sectional designs that rely upon between-person averages across samples at one measurement time point (Lambert et al., 2004). However, in essence, these findings are addressing a different question than the current study; that is, whether globally recalled coping frequency is related to occupational tenure. This dissertation, on the other hand, examined week-to-week coping frequency, which is more of a momentary assessment of the relationship between coping frequency and occupational tenure. The difference between these two questions can be summarized according to the debate of coping being either trait-based (relatively stable across situations) or state-based (fluctuating across situations), which have both received support in the literature (e.g., Compas et al., 1988; Costa et al., 1996; DeLongis \& 
Holtzman, 2005; Todd et al., 2004). Therefore, whereas these prior studies have found support for an effect of occupational tenure on reports of global coping (trait-based relationship), the current study produced no evidence of an association between occupational tenure and weekly ratings of coping frequency (state-based relationship).

This is important for a few reasons. First, the literature on retrospective recall suggests that global reports of coping are poor indicators of actual coping behaviors individuals engage in on a regular or daily basis (Schwarz et al., 1999; Stone et al., 1998; Todd et al., 2004). For instance, Todd et al. (2004) found that global retrospective recall of coping had low concordance with reports of coping gathered on a daily basis, suggesting that fluctuations inherent in coping behaviors are not captured using global retrospective approaches. In the light of this literature, the current study's null findings suggest that occupational tenure does not have a week-to-week effect on coping frequency. Second, related to this point, the current study adopted a different methodological approach than most previous studies, assessing coping frequency over several time points rather than one cross-section of time. Longitudinal data are essential to identifying cause-and-effect relationships (de Lange et al., 2003), making them particularly important when examining relationships between variables. As the current study investigated the effect of occupational tenure over several time points, the lack of evidence is likely a more robust and representative finding than previous studies relying upon one-time assessments. As such, this study suggests that occupational tenure does not seem to cause a change in coping over time. 
Finally, there is also evidence that other tenure variables such as organizational tenure might explain coping frequency. Indeed, the significant positive correlation between organizational tenure and emotion-focused coping frequency are supportive of a relationship between these two variables. Although this is inconsistent with most studies investigating the relationship between occupational tenure and coping (Lambert et al., 2004), a few studies have found an association between organizational tenure and emotion-focused coping, though it is usually negative (Havlovic \& Keenan, 1995; Sundberg, 2003). The current study suggests, however, that as organizational tenure increases, emotion-focused coping also increases. This may be due to nurses becoming involved in conflicts that are more difficult to resolve as they gain tenure in the organization, which may require the use of emotion-focused coping to deal with them. This is consistent with literature suggesting that more emotion-focused coping is used more when problem-focused coping is unable to resolve the stressful situation (Tennen et al., 2000). However, organizational tenure failed to predict coping frequency, despite the significant positive correlation with emotion-focused coping frequency. Therefore, although there may be an association between organizational tenure and increases in emotion-focused coping frequency, the results suggest this is small.

\section{Coping Frequency Variation}

Contrary to my prediction, this dissertation offers no evidence to support a relationship between occupational tenure and person coping frequency or, in other words, fluctuations in using different coping strategies by person (Research Question 1). This suggests that on the person level, occupational tenure does not affect whether nurses vary 
their coping strategies to deal with WIC. However, the results from the supplemental analysis suggest that as organizational tenure increased, rather than occupational tenure, nurses demonstrated a tendency toward using certain coping strategies more than others at both the person level and at the weekly level. That is, nurses used specific coping strategies to deal with WIC, or a differentiated coping approach, rather than using all or most of the strategies. These are intriguing findings, which have not been identified in the previous literature.

Organizational tenure was an important positive predictor of person coping variation and weekly coping variation. At the level of the person, greater coping frequency variation suggests a differentiated coping style (trait-coping), wherein the person has adopted a general coping pattern that consists of specific coping strategies used to deal with stressors such as WIC across situations (e.g., Atkinson \& Violato, 1994; Lu, 1996; Schwartz et al., 1999). Therefore, this finding suggests that organizational tenure affects how much a person fluctuates in their global approach to coping with WIC. At the level of the conflict situation, greater coping frequency variation is reflective of the person's differential choice of coping strategies to deal with the particular conflict, likely based upon characteristics of the situation, as put forth by the matching hypothesis and state-coping advocates (e.g., DeLongis \& Holtzman, 2005; Lazarus, 1993; Lazarus \& Folkman, 1984). These interpretations suggest that both trait- and state-coping mechanisms are in operation, which is consistent with other prior research (Frydenberg \& Lewis, 1994; Jorgensen et al., 2009). Therefore, in the context of the current study, it seems that as an individual becomes more accustomed to aspects of the organization, the 
job, and the people worked with, the individual may adopt certain coping patterns for WIC and yet, still attempt to match coping efforts to specific characteristics of conflict situations. However, this does not necessarily mean that more organizational tenure leads the individual to more effectively cope with the conflict, since this direct relationship was not supported by the results.

Supplemental analyses of the relationship between coping frequency variation and coping effectiveness, however, suggest that organizational tenure may have an indirect influence on coping effectiveness through coping frequency variation. Indeed, both person and conflict situation coping frequency variation was positively predictive of coping effectiveness. This may be due to the fact that using less coping strategies (differentiated coping) does not waste effort on those strategies which are not effective for that person or strategies the person is not comfortable or adept at using. Using fewer coping strategies may also be effective simply because the individual only uses those strategies that have proven effective for them in the past and the individual has learned, from among these strategies, those that are best to address specific characteristics of conflicts.

Caution in interpreting these findings is warranted. Indeed, using fewer coping strategies may not always be superior, since employing less coping strategies effectively might suggest the stressor was easily resolved or using more coping strategies less effectively may be indicative of an extremely difficult stressor to overcome (Tennen et al., 2000). For example, an individual may initially attempt to overcome a challenging stressful conflict with a colleague by making problem-focused coping efforts. These 
could potentially fail in bringing a resolution to the conflict stressor due to the other party's unwillingness to respond, requiring the individual to then employ emotionfocused coping. Therefore, although these efforts may ultimately produce ineffective results, it may be due to outstanding characteristics of the conflict rather than the coping behavior itself. In general, however, these results suggest that using fewer coping strategies to deal with WIC is better than using many coping strategies.

Within the literature, using a variety of coping strategies to cope (coping versatility), is generally related to increased coping effectiveness (Mattlin et al. 1990; Pearlin \& Schooler, 1978), contrary to the findings from the current study. However, these findings emerged under conditions of coping with chronic stressors, where some coping strategies have likely failed to alleviate distress initially (e.g., problem-focused coping) and the individual must turn to other strategies (i.e., emotion-focused coping); thereby this finding in the literature may be more representative of characteristics of the stressor rather than the actual effectiveness of the coping itself (Tennen et al., 2000). Reconciling the differences between this study and these other studies is further aided by considering each instance of WIC as an everyday stressor, as opposed to varying levels of pain or physical symptoms (in the case of a chronic stressor), which are ongoing and require many coping approaches. On one hand, using the coping strategy the individual uses the most and is likely best able to effectively employ would be best for everyday stressors, which is consistent with a coping trait perspective (Vaillant, 1977). Or, this coping response should be the coping strategy that best matches the characteristics of the WIC stressor, which is consistent with state-coping such as advocated by the 
transactional model and matching hypothesis (Lazarus, 1993; Lazarus \& Folkman, 1984). Therefore, using fewer coping strategies is likely more effective than using more coping strategies because the individual is better able to effectively use the coping strategy or match the coping effort to characteristics of the stressor.

Taken together, therefore, the positive effect organizational tenure had on both person and weekly coping frequency variation, and the positive effect these coping frequency variation variables had on coping effectiveness provide a new finding for this literature. Interestingly, these results suggest that as individuals gain experience within their organization, they differentiate their coping or use certain coping strategies more than others to deal with WIC, which then leads to increases in coping effectiveness. This lends some support to the applicability of both the coping trait perspective ( $\mathrm{Lu}, 1996$; Valliant, 1977) and the matching hypothesis within organizations (Lazarus, 1993; Lazarus, 2000). For instance, employees may be adopting coping patterns that they prefer and that are effective for them in dealing with WIC. Also, within these broad coping patterns, it seems that the matching hypothesis is also supported as individuals likely seek to match their coping efforts to characteristics of the situation, yielding increases in effective outcomes. Therefore, the differentiated coping efforts of those with greater organizational tenure suggest that employees are using their own preferred coping style while potentially matching coping efforts to specific stressors rather than using many coping strategies. As such, coping is likely most effective when employees develop personal coping repertoires of ways to deal with WIC and, from this coping repertoire, 
make specific coping efforts matched to characteristics of the conflict; and this may be easier to adopt as one gains experience within an organization.

\section{Other Findings}

Although this was not a hypothesized relationship, it is interesting to note that the number of hours worked per week had a strong negative relationship with coping effectiveness, suggesting increases in hours worked per week diminished the positive impact of one's coping efforts. This may be due to two potential mechanisms: 1) time away from work may be a way to cope with WIC in and of itself; and 2) time away from work replenishes critical resources that enable effective coping. The first explanation is consistent with the idea that avoidance coping strategies (e.g., behavioral disengagement, mental disengagement, absenteeism) provide the individual with a needed break or a "psychological breather" from the stressor or stressful environment (Carver et al., 1993; Hardy et al., 2003). Therefore, if an individual is unable to take a sufficient break due to increased hours of work, the individual may not be able to engage in avoidance coping strategies which could otherwise be effective.

Even more likely, time away from work often allows the individual to begin to replenish resources that have been depleted at work. This is most consistent with work effort recovery models (Meijman \& Mulder, 1998), which posit that individuals exert effort at work that result in an accumulation of aversive psychological, behavioral, and subjective conditions. Once effort ceases, however, the individual begins to recover, making time away from work a critical time in which to recover from the efforts expended during work. Moreover, the Conservation of Resources Theory (Hobfoll, 1989; 
Hobfoll \& Lilly, 1993) further proposes that an individual's resources are drained much more quickly than replenishment can take place, suggesting that more time away from work is needed to recover from the loss of resources inherent in working. As such, when individuals work more hours than what they are accustomed to, they expend resources and have less time away from work to recover resources that enable them to effectively cope with WIC. In other words, these results suggest that in order for coping to have the intended effective impact on dealing with WIC, employees need sufficient time away from work to mobilize and replenish their resources.

As an example to illustrate this, one could consider self-control as a mechanism of emotion-focused coping since this strategy inherently focuses on regulating one's emotional reaction to events (stressors) such as WIC. Self-control has been compared to a muscle, where regular exercise or use can increase the strength and ability of self-control to be effectively employed across multiple different scenarios (Baumeister et al., 1998; Muraven \& Baumeister, 2000). However, much the same as a muscle, if self-control is utilized too much or too often without a rest or recovery period, self-control can become depleted and eventually fail in times of need. As an individual works more than normal, he/she will likely have to use self-control as a coping mechanism across many different work stressors. Then, when exposed to WIC, this may require the further use of selfcontrol, which may fail as a strategy to effectively cope with WIC if it has been depleted as a resource already through too frequent use or insufficient time away from work to recover. Finally, in relation to this finding, it is important to note that this emerged despite only having hours worked per week on the person level (one estimate for each 
nurse). Therefore, in future research, it will be important to verify these findings over time using actual weekly estimates of hours worked per week.

\section{Theoretical Implications}

First, the results of this within-persons study, coupled with the results in the literature, suggest that the goodness of fit hypothesis may be context-dependent. That is, the predicted relationships between control and coping are likely valid only under certain contextual conditions. This interpretation ties together the null findings from the current study with the inconsistent support for the goodness of fit hypothesis in the literature (e.g., Kendall \& Terry, 2008; Park et al., 2004). Based on the results from this study, the underlying mechanism of matching perceived control and coping efforts is in operation among nurses, though this did not produce beneficial outcomes. This suggests that certain between-persons variables may be responsible for goodness of fit among nurses such as demographic individual differences. Given this is the first study conducted within the workplace on goodness of fit to be the best of my knowledge, it is also possible that the workplace may not be an appropriate context for goodness of fit. This interpretation is unlikely, however, given the number of findings that are generally consistent with the predictions of the goodness of fit hypothesis in the workplace (e.g., Hahn, 2000; Portello \& Long, 2001; Zapf \& Gross, 2001). The results of this dissertation imply that the goodness of fit hypothesis is not applicable in every situation, but that systematic indepth testing of the hypothesis is needed to discover applicable contexts.

Although the goodness of fit predictions were not supported, this study suggests that control and coping, independently, are important variables in determining the 
effectiveness of coping in the workplace, particularly in nursing. Consistent with the literature on control (e.g., Karasek, 1979; Theorell, 2003), this study suggests that increased control is important for coping efforts to be effective, suggesting control has an important role in coping effectively with nursing stressors. Moreover, these results support coping, irrespective of whether it is problem- or emotion-focused coping, as an effective way to deal with WIC. More specifically, any type of coping seems to be an essential component of successfully mitigating the negative psychological effects of nursing WIC. Therefore, although the interaction of perceived control and coping do not affect coping effectiveness within the nursing workplace, both control and coping are independently important in effectively dealing with nursing WIC.

Although the results of this study are contrary to my proposition that occupational tenure would be important, the finding that organizational tenure, or the amount of time one has spent employed within an organization, is an important predictor of coping supports the argument that goodness of fit is context dependent. Indeed, rather than knowledge of and experience in nursing, knowledge and experience nursing at a specific hospital and with specific individuals in that context is much more important for coping with WIC. This is consistent with the conflict management literature, which specifies certain conflict management techniques for conflicts with different individuals, or referents (Lee, 2002; Ma, Lee \& Yu, 2008; Rahim, 1986). This would suggest that coping with WIC is highly relational, such that nurses need to develop a way of interacting with specific colleagues, as opposed to using the same strategies regardless of the situation or relationship. This interpretation is consistent with both the nursing (Duddle \& Boughton, 
2007) and general interpersonal literatures (Kenny, Mohr \& Levesque, 2001). Therefore, in the context of coping with interpersonal conflicts, effective coping may rely upon characteristics of the dyadic relationship.

Finally, perhaps the most compelling findings of this dissertation, coping frequency variation, on both the person and situation levels, are important positive predictors of coping effectiveness. Greater coping frequency variation, or using fewer coping strategies, directly influenced the effectiveness of coping strategies used to deal with WIC. At the person level, this suggests that a differentiated coping style, or adopting a coping style where fewer coping strategies are used, is a more effective way to cope with WIC, which is generally supportive of the literature on coping as a trait-like phenomenon (e.g., Compas et al., 1988; Lu 1996). Greater coping frequency variation at the weekly or situation level, was even more strongly predictive of effective coping outcomes, suggesting that individuals who used select coping strategies for certain conflicts reported more effective outcomes, which is consistent with the matching hypothesis (e.g., Lazarus, 1993; Lazarus \& Folkman, 1984). Therefore, this dissertation found evidence of benefits for both trait-based and situation-based coping frequency variation.

\section{Practical Implications}

This dissertation focuses on coping with a common stressor (WIC) within a prominent occupational group, thereby offering several practical implications. First, the results suggest that control and coping are both important factors in coping effectively with WIC. Indeed, ways to empower nurses, and potentially other employees, with an 
increased sense of control over WIC may help improve individual coping efforts. For example, offering organizational services such as workshops with specific information to navigate a particular hospital work setting or creating a system that fosters open communication with upper management (e.g., Parker et al., 2001; Richardson \& Rothstein, 2008; Spector, Fox, \& Domagalski, 2006; Thomas \& Ganster, 1995) may help employees feel like they have an increased amount of control over the conflict and associated outcomes. Also, the results of this dissertation suggest that employees confronted with WIC will benefit from any type of coping they engage in, regardless of whether it is problem- or emotion-focused coping. Therefore, rather than pointing to a specific type of coping as more effective in dealing with WIC, these results simply recommend that coping in some way is better than no coping. This seems particularly true in the context of nursing.

In this dissertation, I found that the length of time one is employed within an organization is more important to coping effectively with WIC than the length of time one has been within the profession. This provides further evidence of the importance of retention for organizations in addition to the obvious economic advantages to reducing turnover. Whereas retention of employees is likely a way to promote increased effectiveness in coping, hours worked per week seems to deter coping efforts. Consistent with the literature regarding the negative effects of work overload (e.g., Leitner \& Resch, 2005) and nursing work staffing schedules (e.g., Aiken, Clarke, Sloan, Sochalski \& Silber, 2002), this finding suggests that when employees are working more than usual or are overworked, coping efforts are not as effective. As such, organizations should seek to 
maintain reasonable workloads and schedules for their employees and avoid making substantial changes in the number of hours employees work.

Indeed, perhaps most importantly, the results of this dissertation imply that things should be done at the level of the organization to help individual nurse employees in their coping efforts, especially since most conflicts occurred between nurses themselves. For example, given the predominate reliance upon healthcare teams in most nursing units (Havens et al., 2010; Makinen et al., 2003), organizations should consider varying the level of organizational experience within these teams. By so doing, those with less experience in the organization could benefit from interactions with and observations of their greater tenured counterparts. However, this should be done with caution as the literature suggests that nurses with greater tenure are more likely to victimize younger, inexperienced nurses (Baltimore, 2006; Meissner, 1999). As such, putting teams together of varied organizational experience should be done in tandem with training nurse managers. This training should make it clear that it is the responsibility of the tenured nurses to train and support the younger nurses rather than give them mundane and menial work tasks. With this sense of responsibility and accountability, the more experienced nurses may also benefit by expressing greater organizational commitment and job satisfaction (Eisenberger et al., 1986) while simultaneously modeling and promoting more effective coping with WIC. Therefore, teams of varying organizational experience coupled with management training is a course of organizational action where each party can benefit. 
Taking this one step further, organizations should also consider setting up formal and informal on-going mentoring programs for their nurse employees that extend beyond initial job orientations. Organizations should seek to pair those with greater organizational tenure with those having less experience in the organization in these mentoring efforts for extended periods of time such as several weeks or months. In formal mentoring contexts, those with greater tenure can verbally share what they have found to be effective methods of coping with difficult interpersonal conflicts that arise in the workplace. However, since some of these coping strategies might have been and continue to be used implicitly by those with greater organizational experience, some nurses may have difficulty verbally expressing or explaining how they cope with stressful conflicts. Therefore, it is also essential to have informal, experiential mentoring and training where those with less tenure can observe model coping behaviors of their tenured counterparts. As an extension of this, organizations should consider training supervisors and managers, who generally have greater organizational tenure than their subordinates, to be more supportive by making themselves available for mentoring and accessible for questions (Karasek \& Theorell, 1990). Indeed, using existing leadership hierarchical structures within the nursing context may be one of the most effective ways to transmit organizational knowledge and model effective coping strategies (e.g., Zohar, 2003).

Related to this, these results provide suggestions for organizational employee stress and coping training. Indeed, employees, especially nurses, seem to benefit from varying their coping responses to WIC by having a developed set of select coping strategies in their repertoire and then matching these coping strategies to particular 
situational characteristics. As a common stressor across organizations, it is virtually certain that employees will be exposed to interpersonal conflicts at work (e.g., de Raeve et al., 2009), regardless of the best organizational efforts that can be made to prevent them from occurring (e.g., policies, sanctions). Therefore, as part of their preventative efforts, organizations should include information on varying coping strategies to deal with specific instances of WIC in stress and coping training. That way, this information has the potential to greatly benefit nearly every employee, especially if preventative efforts fail. Through dissemination of these findings to employees through training, especially those who are exposed to frequent work stressors, employee stress and strain outcomes can potentially be substantially reduced. And, by so doing, the health and wellbeing of the organization should increase through the betterment of the health and wellbeing of its employees.

\section{Potential Limitations}

Certain aspects of this study may have limited my ability to examine the relationships among the different components of my hypotheses. First, participants were asked to specify a specific workplace interpersonal conflict they experienced during the week. Although the literature validates this approach in assessing coping efforts (Hahn, 2000), participants may still be subject to biased retrospective recall in their reconstructions of their actual coping behavior. As such, responses regarding WIC could have been biased according to participant retrospection. Moreover, in identifying a specific workplace interpersonal conflict, participants were instructed to report on the most negative or stressful experience for the week. As such, results may only reflect the 
coping process in relation to the most stressful workplace interpersonal conflict and not be representative of coping with all workplace interpersonal conflicts. However, the stress literature suggests examining more extreme situations can produce generalizeable results for less extreme cases (Marco, Neale, Schwartz, Shiffman, \& Stone, 1999), making this unlikely.

Another possible limitation of this design is due to the coping process not being examined within specific conflicts over time. This makes conclusions about the coping process difficult, as inferences have to be made when considering coping throughout the entire stressful experience from inception to resolution. Moreover, the current study did not control for prior history or experience in coping with WIC, as this could affect coping efforts and coping effectiveness in dealing with WIC. Related to this, the current investigation did not consider potential non-independence of the data in regards to the level of the hospital or nursing unit, as ratings of coping effectiveness could potentially be more similar between nurses in the same hospital or unit than between others. Another methodological limitation is the use of a single item measure of perceived control in each instance of WIC. Although the literature has suggested that one-item measures are generally acceptable for psychological factors that are not complex (Wanous et al., 1997), there is a threat of unreliability, as an aggregate across multiple items cannot be computed. Despite these potential limitations, this dissertation examined coping with different cases of WIC over time within nurses, allowing an investigation of the withinperson coping process and strengthening inferences that can be made regarding the coping process. 
This study focused exclusively on self-report data, which are heavily influenced by individual perceptions. Indeed, participants were instructed to report on their perceptions of control, coping efforts and subsequent perceived coping effectiveness within the weekly surveys. The reliance upon self-report data is a potential limitation, as participants can be subject to such problematic biases as social desirability and response set (Ware, 1978). However, the literature has clearly established the important role that perceptions play in the coping process and suggest that to avoid or circumvent them is to overlook critical aspects of coping (Lazarus, DeLongis, Folkman, \& Gruen, 1985). Indeed, consistent with the transactional model, I argue that if the individual perceives that a coping effort has been effective, regardless of objective conditions, that the coping effort can be considered effective. Thus, despite the heavy reliance upon self-report perception data, the coping process is likely to be accurately captured.

Some characteristics of the data are also potential limitations in this study. First, missing data analyses suggested that occupational tenure was negatively related to weekly survey completion. As such, these data were skewed towards those with greater occupational tenure and likely not representative of all nurses in the sample, particularly those newer to the profession, potentially restricting my ability to examine some of my hypotheses. Related to this, weekly contrast analyses also demonstrated that participants reported greater coping frequency and weekly coping variation in the beginning of the study. This may be indicative of study fatigue, where participants systematically provide less data over time. Although this is one plausible interpretation, coping effectiveness 
scores did not differ significantly across weeks, providing some support that study fatigue was not the likely cause.

Furthermore, there is some question about my measure of coping. First, internal consistency estimates were a bit low, suggesting that the items used may represent the same factor (i.e., problem-focused coping, emotion-focused coping). This is a concern, as latent factors should have items that are consistent with each other to ensure the factor is adequately represented by the data. Second, the unbalanced number of items used to represent problem- and emotion-focused coping is a potential problem. Although this is common within the coping literature (e.g., Folkman \& Lazarus, 1980; Park et al., 2004), this also represents a potential threat to accurate conceptualization of these coping constructs. Moreover, removal of humor coping from the 2-factor model deleted approximately one-eighth of the data and may have limited the representativeness of the emotion-focused coping construct. However, by omitting humor coping, model fit estimates significantly improved, suggesting that this was the correct choice for modeling the data to represent the common construct of emotion-focused coping. Lastly, many of the multilevel models had substantial missing data in the chi-square significance testing of random effects (i.e., variance components or slopes), which can make obtaining significant results difficult. Therefore, these tests may have been limited in the ability to detect significant differences in slopes between persons.

Finally, I used a sample of nurses for this dissertation and this may or may not be representative of other populations. For instance, average occupational tenure of my sample was substantially greater than the general ONA membership, suggesting that the 
sample lacked early career nurses and, therefore may not be representative. Additionally, my sample is primarily composed of women, which may affect the coping process. Indeed, the literature suggests that gender differences exist in coping frequency and coping effectiveness such that women tend to use emotion-focused coping more often than men and use these strategies more effectively than men (e.g., Porter et al., 2000). Moreover, nurses may not be representative of other occupational groups due to other factors such as educational training and work schedules, which vary considerably across occupations. However, as nurses are an occupational population exposed to frequent and intense conflicts with those at work (e.g., Baltimore, 2006), they likely represent a wide array of occupations in respect to coping with WIC. Moreover, participants in this sample were taken from both rural and urban institutions, thereby further strengthening the generalizations that can be made to other nurses and occupations.

\section{Strengths and Future Research}

The strengths of this dissertation provide future research with a foundation upon which to explore several further avenues related to coping and interpersonal conflict. First, despite several studies being devoted to examining coping effectiveness and the goodness of fit hypothesis, the literature has continued to lack an investigation of the goodness of fit hypothesis within a workplace context. Therefore, this gap in the

literature has been addressed by this dissertation. However, since this was done in a specific context, that of nursing, future research should consider examining the goodness of fit hypothesis within other workplace contexts. Indeed, although goodness of fit does 
not seem to apply to nursing, the interactions between perceived control and coping efforts could be very important within other workplace contexts such as law enforcement, public teaching, or retail. Furthermore, future research should explore how such variables as organizational tenure, hours worked per week, and coping variation might affect goodness of fit predictions within these other occupational contexts. Of additional importance, future investigations should consider the role of personality in goodness of fit; that is, include such individual difference variables as locus of control, self-efficacy, neuroticism, optimism, or openness to experience. By so doing, future research can uncover contexts in which goodness of fit is valid and provide continued explanation for the inconsistencies within this literature (Kendall \& Terry, 2008; Park et al., 2004).

As the focal stressor, this dissertation examined coping with workplace interpersonal conflict. Social stressors, such as interpersonal conflict, are some of the most difficult and troublesome daily stressors (Bolger et al., 1989; Smith \& Sulsky, 2007) and also cost organizations a great deal of money (Dana, 1999; Keenan \& Newton, 1985). Moreover, employees within virtually any type of occupation will interact with others at some point and potentially have interpersonal conflicts, making WIC a subject of great practical importance. Future research should continue to investigate coping with WIC and other significant social stressors within other occupational settings. By so doing, this can produce substantial benefits for both the employee and the organization, maximizing benefits of effective coping. Moreover, future research should investigate ways that coping effectively with social stressors can be transferred into coping effectively with other workplace stressors such as work overload or difficult work 
structures. Extending upon this further, studies could be done where different problemfocused coping efforts could be compared to see if one type of problem-focused coping is more effective than others, precluding the need to use other forms of problem-focused coping and explain the typical low internal consistencies for latent factor structures; this could also be done for emotion-focused coping. These research avenues can potentially produce very valuable information that can be practically relevant to difficult and common work problems.

Of additional practical importance, this dissertation examined coping with WIC within nursing, which has been identified as a particular challenge for this population (e.g., Almost, 2006; Baltimore, 2007). Indeed, nurses have elevated rates of conflict among themselves and with other parties in the healthcare system (Farrell, 1999). Future research should continue to explore ways that nurses can mitigate the harmful effects of conflict including additional ways of coping such as proactive (Aspinwall \& Taylor, 1997) or anticipatory coping (Folkman \& Lazarus, 1985) and conflict management strategies (Blake \& Mouton, 1964; Ma, Lee \& Yu, 2008; Rahim, 1986) in preventing WIC. Moreover, future research should also investigate the potential non-independence of coping between-persons by examining more complex multilevel models that include additional levels of analysis such as hospital organization or nursing unit. Furthermore, continued exploration of ways in which to foster good relationships between health professionals, managers and patients/patient families may be another fruitful area for development among nurses, especially given the importance of interpersonal relationships in patient quality of care (Havens, Vasey, Gittell \& Lin, 2010). Improving 
communications between parties such as between nurses and physicians, for example (Narasimhan, Eisen, Mahoney, Acerra, \& Rosen, 2006), could be one avenue to investigate.

Furthermore, this dissertation sought to produce information that could empower nurses and other employees with information regarding ways to effectively cope with WIC. However, rather than focusing on what nurses can do to resolve interpersonal conflicts at work, future intervention research should also explore how organizations can enact policies and procedures that could effectively reduce conflict among nurses and within other occupational groups. Despite recent reviews within the work stress literature suggesting that occupational stress interventions employed at different levels (e.g., individual, organizational) are more effective (Lamontagne, Keegel, Louie, Ostry, \& Landsbergis, 2008; Richardson \& Rothstein, 2008), the nursing literature continues to focus on individual-level interventions (e.g., Boey, 1999; Lambert et al., 2003; Zimber, Rudolf, \& Teufel, 2001). By taking an approach that incorporates both the individual level (coping) and the organizational level (policies), conflict is much more likely to be avoided or prevented and the well-being of both the employees and the organization should improve as a result.

In accordance with the recommendations in the literature (e.g., Park et al., 2004), this dissertation employed a rigorous methodological approach, a within-persons technique (multilevel or hierarchical linear regression) to examine coping with stressors. Future research testing the goodness of fit hypothesis and other coping-related hypotheses should continue to use this method, which offers the unique ability to 
examine relationships both between- and within-persons, allowing in-depth analysis. Of additional consideration is the use of longitudinal data to examine not only coping with stressors between- and within-persons, but also within-stressors, which can shed light on the coping process itself. In other words, future research should consider coping with interpersonal conflict stressors from inception to resolution in order to uncover the specific process that individuals undergo, which may vary considerably over time within stressors. Multilevel regression techniques are uniquely able to handle these types of research questions and should be employed to examine such phenomena (de Lange et al., 2003; Raudenbush \& Bryk, 2002).

Finally, the results of this dissertation suggest that coping variation is important in determining the effectiveness of coping efforts. This relationship may be due to individuals using only those coping strategies they are comfortable with or adept at using in dealing with WIC and matching specific characteristics of the situation/stressor with specific coping efforts. This explanation suggests that both trait-based and situationbased coping is occurring, which is consistent with the literature (e.g., Frydenberg \& Lewis, 1994; Jorgensen et al., 2009). However, future research should consider ways in which to more closely examine whether coping variation is more of a trait or state in the coping process. Furthermore, intervention research could explore the relative efficacy of training individuals on coping variation to explore whether this may be another way to promote more effective coping. Finally, other person-level and situation-level variables should be explored in relation to coping variation and coping effectiveness. Such variables as source of interpersonal conflict (Frone, 2000) and mood/emotions (Lazarus, 
2006; Marco et al., 1999) have been demonstrated in the literature as important factors in the stress and coping process and should be examined relative to coping variation and coping effectiveness.

\section{Conclusion}

In conclusion, this dissertation sought to elucidate the process of coping effectively with WIC in the nursing workplace by taking a within-persons approach in testing the goodness of fit hypothesis. The results of this dissertation suggest that nursing is not a context in which goodness of fit is valid and do not support occupational tenure as an important variable in coping with WIC. However, problem-focused and emotionfocused coping, perceived control, organizational tenure, hours worked per week and coping frequency variation were all significant factors in determining the effectiveness of coping. Perhaps most importantly, these results highlight coping frequency variation as a particularly important strategy to increase the effectiveness of one's coping efforts. These results can be used to inform future research on the goodness of fit hypothesis and empower nurses and other employees to more effectively cope with WIC and other workplace stressors. Finally, this dissertation offers practical suggestions on ways to more effectively cope with conflict, which can lead to improvements in health and wellbeing in the increasingly difficult work conditions the contemporary workplace and health care contexts embody. As such, it is my hope that these results can be used to advance both future research and practical applications within nursing and a variety of occupational contexts. 
Table 1: Comparison of Model Fit across 2-factor and 3-factor Model Variants

\begin{tabular}{|c|c|c|c|c|c|}
\hline & $\begin{array}{l}\text { 2-Factor } \\
\text { Model } \\
\text { All items }\end{array}$ & $\begin{array}{l}\text { 2-Factor } \\
\text { Model } \\
\text { RC } \\
\text { omitted }\end{array}$ & $\begin{array}{l}\text { 2-Factor } \\
\text { Model RC } \\
\text { and HC } \\
\text { omitted }\end{array}$ & $\begin{array}{l}\text { 2-Factor } \\
\text { Model HC } \\
\text { omitted }\end{array}$ & $\begin{array}{l}\text { 3-Factor } \\
\text { Model } \\
\text { HC } \\
\text { omitted }\end{array}$ \\
\hline \multicolumn{6}{|l|}{ Absolute Fit Indices } \\
\hline $\begin{array}{l}\text { Chi-Square (degrees of } \\
\text { freedom) }\end{array}$ & $87.45(19)$ & $72.75(13)$ & $23.09(8)$ & $38.14(13)$ & $13.30(6)$ \\
\hline Hoelter's CN & 178 & 164 & 374 & 312 & 380 \\
\hline $\begin{array}{l}\text { Akaike's Information } \\
\text { Criteria (AIC) }\end{array}$ & 137 & 117 & 61 & 82 & 75 \\
\hline \multicolumn{6}{|l|}{ Relative Fit Indices } \\
\hline $\begin{array}{l}\text { Normed Fit Index } \\
\text { (NFI) }\end{array}$ & .74 & .77 & .91 & .86 & .90 \\
\hline $\begin{array}{l}\text { Incremental Fit Index } \\
\text { (IFI) }\end{array}$ & .78 & .80 & .94 & .90 & .94 \\
\hline \multicolumn{6}{|l|}{$\begin{array}{l}\text { Noncentrality Fit } \\
\text { Indices }\end{array}$} \\
\hline $\begin{array}{l}\text { Root Mean Square } \\
\text { Error of Approximation } \\
\text { (RMSEA) }\end{array}$ & .09 & .10 & .07 & .06 & .06 \\
\hline $\begin{array}{l}\text { Comparative Fit Index } \\
\text { (CFI) }\end{array}$ & .77 & .79 & .94 & .90 & .93 \\
\hline
\end{tabular}

Note: $N=406 ; \mathrm{RC}=$ Religious Coping; $\mathrm{HC}=$ Humor Coping 
Table 2: Means and Standard Deviations of the Individual Weekly Coping Frequency and Coping Effectiveness Variables

\begin{tabular}{|l|c|c|}
\hline & Mean & Standard Deviation \\
\hline Perceived Control & .83 & 1.00 \\
\hline Active Coping Frequency & 2.35 & 1.41 \\
\hline Positive Reframing Frequency & 1.58 & 1.33 \\
\hline Humor Coping Frequency & .79 & 1.18 \\
\hline Religious Coping Frequency & .52 & 1.12 \\
\hline Emotional Social Support Frequency & 1.56 & 1.36 \\
\hline Mental Disengagement Frequency & 1.77 & 1.43 \\
\hline Behavioral Disengagement Frequency & 1.22 & 1.38 \\
\hline Instrumental Social Support Frequency & 1.51 & 1.41 \\
\hline Active Coping Effectiveness & .84 & .65 \\
\hline Positive Reframing Effectiveness & .68 & .62 \\
\hline Humor Coping Effectiveness & .84 & .64 \\
\hline Religious Coping Effectiveness & 1.05 & .62 \\
\hline Emotional Social Support Effectiveness & 1.05 & .58 \\
\hline Mental Disengagement Effectiveness & 1.03 & .53 \\
\hline Behavioral Disengagement Effectiveness & .62 & .62 \\
\hline Instrumental Social Support Effectiveness & .99 & .59 \\
\hline
\end{tabular}

Note: $N=143$. Range of coping frequency items was 0 to 4 and 0 to 2 for coping effectiveness items. All values are aggregated across weeks and individuals. Therefore, whereas the mean value of 2.35 for active coping frequency suggests that active coping, on average, was used quite often across individuals and weeks in the study, the mean value of .52 for religious coping frequency implies that religious coping, on average, was used infrequently across individuals and weeks. Coping effectiveness values can be interpreted similarly. 
Table 3: Complete Correlation Matrix for Demographic and Study Variables Across Level 1 and Level 2

\begin{tabular}{|c|c|c|c|c|c|c|c|c|c|c|}
\hline & $M(S D)$ & Age & $\begin{array}{c}\text { Hours } \\
\text { Worked }\end{array}$ & $\begin{array}{l}\text { Occ } \\
\text { Tenure }\end{array}$ & $\begin{array}{l}\text { Org } \\
\text { Tenure }\end{array}$ & Control & $\begin{array}{l}\text { Problem- } \\
\text { Focused } \\
\text { Coping }\end{array}$ & $\begin{array}{l}\text { Emotion- } \\
\text { Focused } \\
\text { Coping }\end{array}$ & $\begin{array}{c}\text { Coping } \\
\text { Effective }\end{array}$ & $\begin{array}{c}\text { Person } \\
\text { Coping } \\
\text { Variation }\end{array}$ \\
\hline Age & $44.36(10.65)$ & 1 & & & & & & & & \\
\hline $\begin{array}{l}\text { Hours } \\
\text { Worked }\end{array}$ & $37.21(9.88)$ & -.03 & 1 & & & & & & & \\
\hline $\begin{array}{l}\text { Occupational } \\
\text { Tenure }\end{array}$ & $16.48(11.81)$ & $.82 * *$ & -.02 & 1 & & & & & & \\
\hline $\begin{array}{l}\text { Organization } \\
\text { al Tenure }\end{array}$ & $11.21(8.82)$ & $.49 * *$ & $-.14 * *$ & $.64 * *$ & 1 & & & & & \\
\hline $\begin{array}{l}\text { Perceived } \\
\text { Control } \\
\end{array}$ & $.83(1.00)$ & .05 & -.01 & .02 & .06 & 1 & & & & \\
\hline $\begin{array}{l}\text { Problem- } \\
\text { Focused } \\
\text { Coping }\end{array}$ & $1.96(1.12)$ & -.03 & .04 & .03 & -.01 & $.16^{* *}$ & 1 & & & \\
\hline $\begin{array}{l}\text { Emotion- } \\
\text { Focused } \\
\text { Coping }\end{array}$ & $1.63(.73)$ & .04 & .01 & $.07 *$ & -.04 & $.06^{*}$ & $.37 * *$ & 1 & & \\
\hline $\begin{array}{l}\text { Coping } \\
\text { Effectiveness }\end{array}$ & $.89(.43)$ & .03 & $-.15^{* *}$ & .05 & -.01 & $.15^{* *}$ & $.24 * *$ & $.31 * *$ & 1 & \\
\hline $\begin{array}{l}\text { Person } \\
\text { Coping } \\
\text { Variation }\end{array}$ & $1.45(.19)$ & -.01 & -.06 & .02 & .15 & -.09 & .05 & -.07 & .16 & 1 \\
\hline $\begin{array}{l}\text { Weekly } \\
\text { Coping } \\
\text { Variation }\end{array}$ & $1.39(.39)$ & .05 & $-.07 *$ & .05 & $.12 * *$ & .05 & $.17 * *$ & .01 & $.22 * *$ & $.45^{* *}$ \\
\hline
\end{tabular}

$* p<.05, * * p<.01$

Note: $N=143$. 
Table 4: Hypothesis 1 Multilevel Regression Results Predicting Weekly Coping Effectiveness from Weekly Control and Coping

\begin{tabular}{|c|c|c|}
\hline & \multicolumn{2}{|c|}{ Coping Effectiveness } \\
\hline Main Effects Model & Fixed Effects & Variance Components \\
\hline Perceived Control & $.03^{\mathrm{a}}(.013)^{* *}$ & .032 \\
\hline Problem-Focused Coping & $.06^{\mathrm{a}}(.015)^{* * *}$ & $.084 * *$ \\
\hline Emotion-Focused Coping & $.08^{\mathrm{a}}(.024)^{* *}$ & .126 \\
\hline Interaction Model & Fixed Effects & Variance Components \\
\hline Perceived Control & $.031^{\mathrm{a}}(.014)^{*}$ & .037 \\
\hline Problem-Focused Coping & $.058^{\mathrm{a}}(.016)^{* * *}$ & $.078 * *$ \\
\hline Emotion-Focused Coping & $.080^{\mathrm{a}}(.025)^{* * *}$ & $.125^{*}$ \\
\hline Control x Problem-Focused Coping & $.001^{\mathrm{b}}(.008)$ & .023 \\
\hline Control x Emotion-Focused Coping & $.019^{\mathrm{b}}(.028)$ & $.110^{\dagger}$ \\
\hline
\end{tabular}

Note: $N=143$. Fixed effects values are the unstandardized within-person partial regression coefficients and robust standard errors are reported in parentheses. Variance component values are reported as the square root of the variance components (standard deviation) for ease of interpretation. Tests of significance for random effects are $\chi^{2}$ with $d f=106$ for the main effects model and $d f=66$ for the interaction model, as only 67 of 137 participants provided sufficient data for computation.

${ }^{\mathrm{a}} d f=138$

$\mathrm{b} d f=137$

${ }^{\dagger} p<.10, * p<.05, * * p<.01, * * * p<.001$ 
Table 5: Hypothesis 2 Multilevel Regression Results Predicting Weekly Coping Effectiveness from Control, Coping, and Occupational Tenure

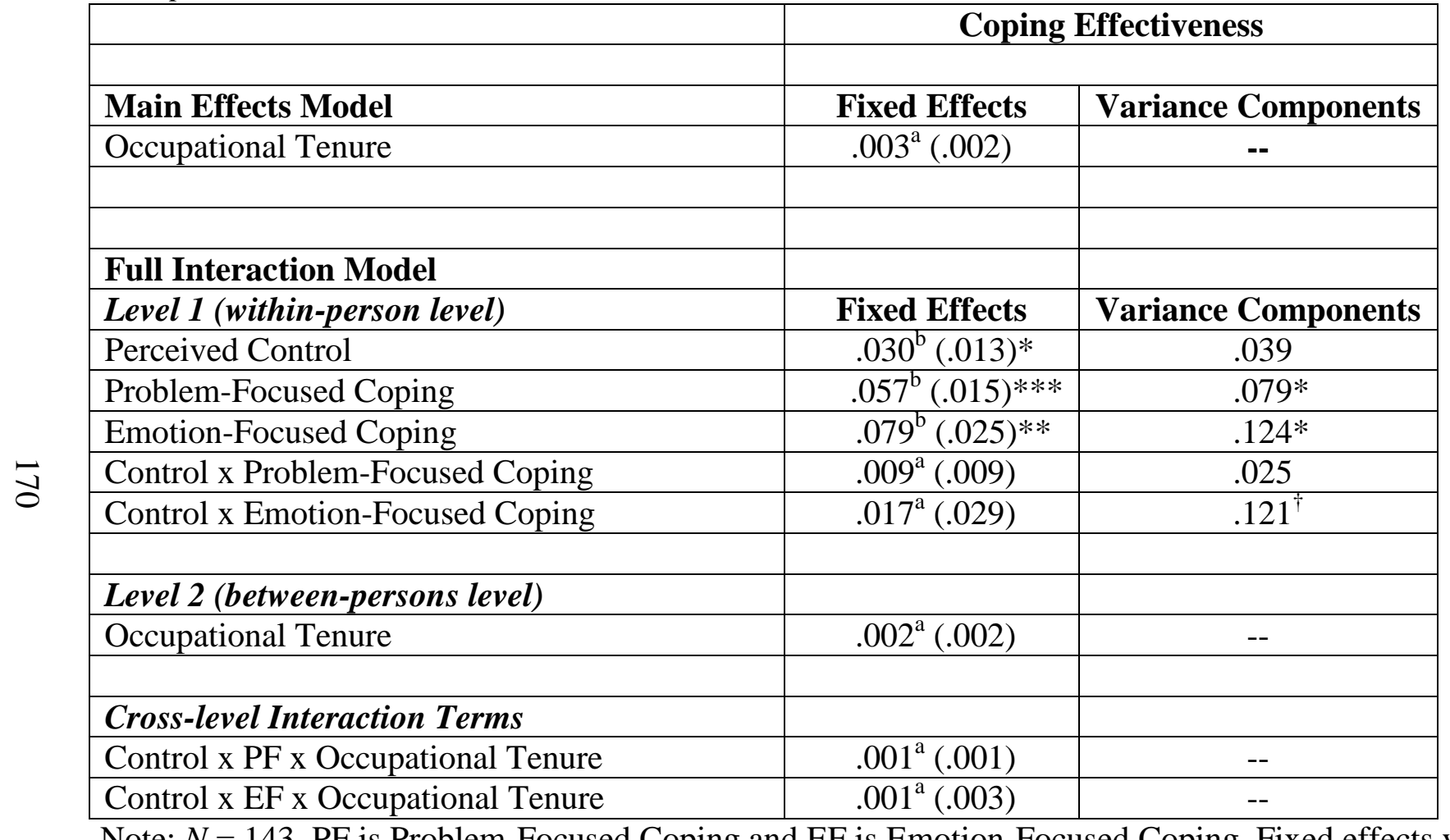

Note: $N=143$. PF is Problem-Focused Coping and EF is Emotion-Focused Coping. Fixed effects values are the unstandardized within-person partial regression coefficients and robust standard errors are reported in parentheses.

Variance component values are reported as the square root of the variance components (standard deviation) for ease of interpretation. Tests of significance for random effects are $\chi^{2}$ with $d f=67$ for Level 1 main effect variables and $d f=66$ for the Level 1 interaction terms, as only 68 of 137 participants provided sufficient data for computation.

${ }^{\mathrm{a}} d f=137$

$\mathrm{b} d f=138$

${ }^{\dagger} p<.10, * p<.05, * * p<.01, * * * p<.001$ 
Table 6: Hypothesis 3, Multilevel Regression Results of Variables Predicting Problem- and Emotion-Focused Coping Frequency as the Only Predictor in the Model

\begin{tabular}{|l|c|c|}
\hline & $\begin{array}{c}\text { Problem-Focused Coping } \\
\text { Frequency }\end{array}$ & $\begin{array}{c}\text { Emotion-Focused Coping } \\
\text { Frequency }\end{array}$ \\
\hline & & Fixed Effects \\
\hline Level 2 (between-persons) Predictor & Fixed Effects & $.004^{\mathrm{a}}(.004)$ \\
\hline Occupational Tenure & $.002^{\mathrm{a}}(.005)$ & \\
\hline
\end{tabular}

Note: $N=143$. Values displayed are the unstandardized within-person partial regression coefficients (fixed effects) and robust standard errors are reported in parentheses. Each Level 2 predictor was grand-mean centered and entered in a unique equation as the only predictor of coping effectiveness. Variance component values are reported as the square root of the variance components (standard deviation) for ease of interpretation. Tests of significance for random effects are $\chi^{2}$ with $d f=$ 119. Weekly contrasts are not presented in the table, though Week 1 and Week 2 had higher levels of problem-focused

$\exists \quad$ coping while Week 1 had higher levels of emotion-focused coping.

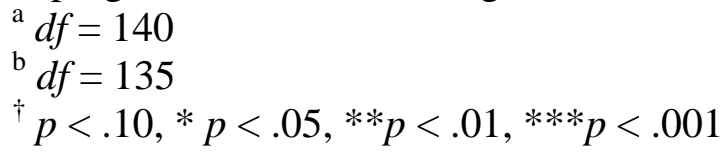


Table 7: Research Question 1, Ordinary Least Squares Regression Results for Occupational Tenure Predicting Person Coping Variation

\begin{tabular}{|l|c|c|c|c|c|}
\hline Predictor (Level 2, between-persons) & $b$ & $t$ & $d f$ & $p$-value & $R^{2}$ \\
\hline Occupational Tenure & .000 & .28 & 140 & .778 & .001 \\
\hline & & & & & \\
\hline
\end{tabular}

Note: $N=143$. Data were aggregated across weeks for this analysis.

${ }^{\dagger} p<.10, * p<.05, * * p<.01, * * * p<.001$ 
Figure 1: A Model of Workplace Interpersonal Conflict

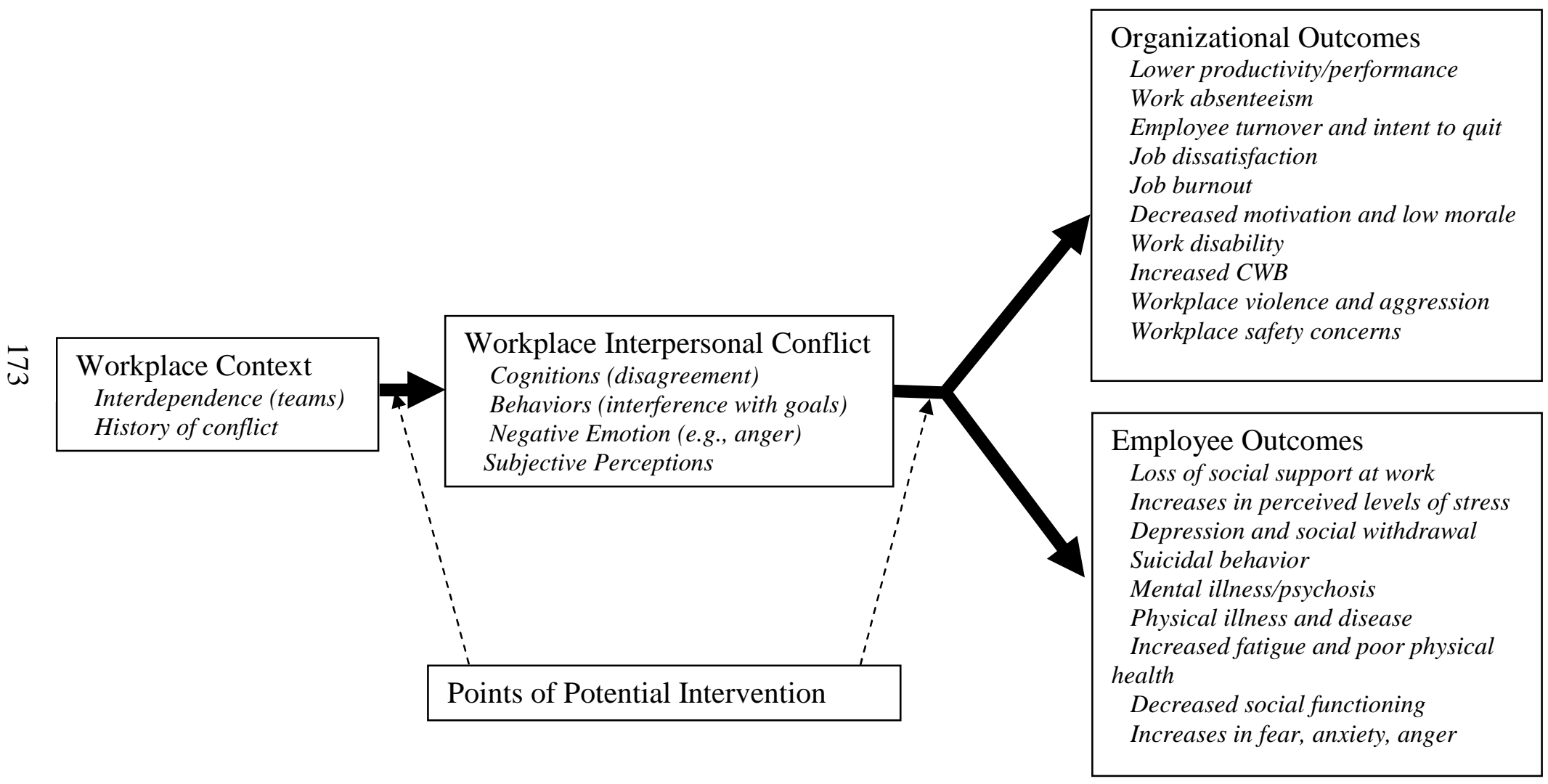


Figure 2: Venn Diagram of the Interpersonal Conflict Construct (Barki \& Hartwick, 2004)

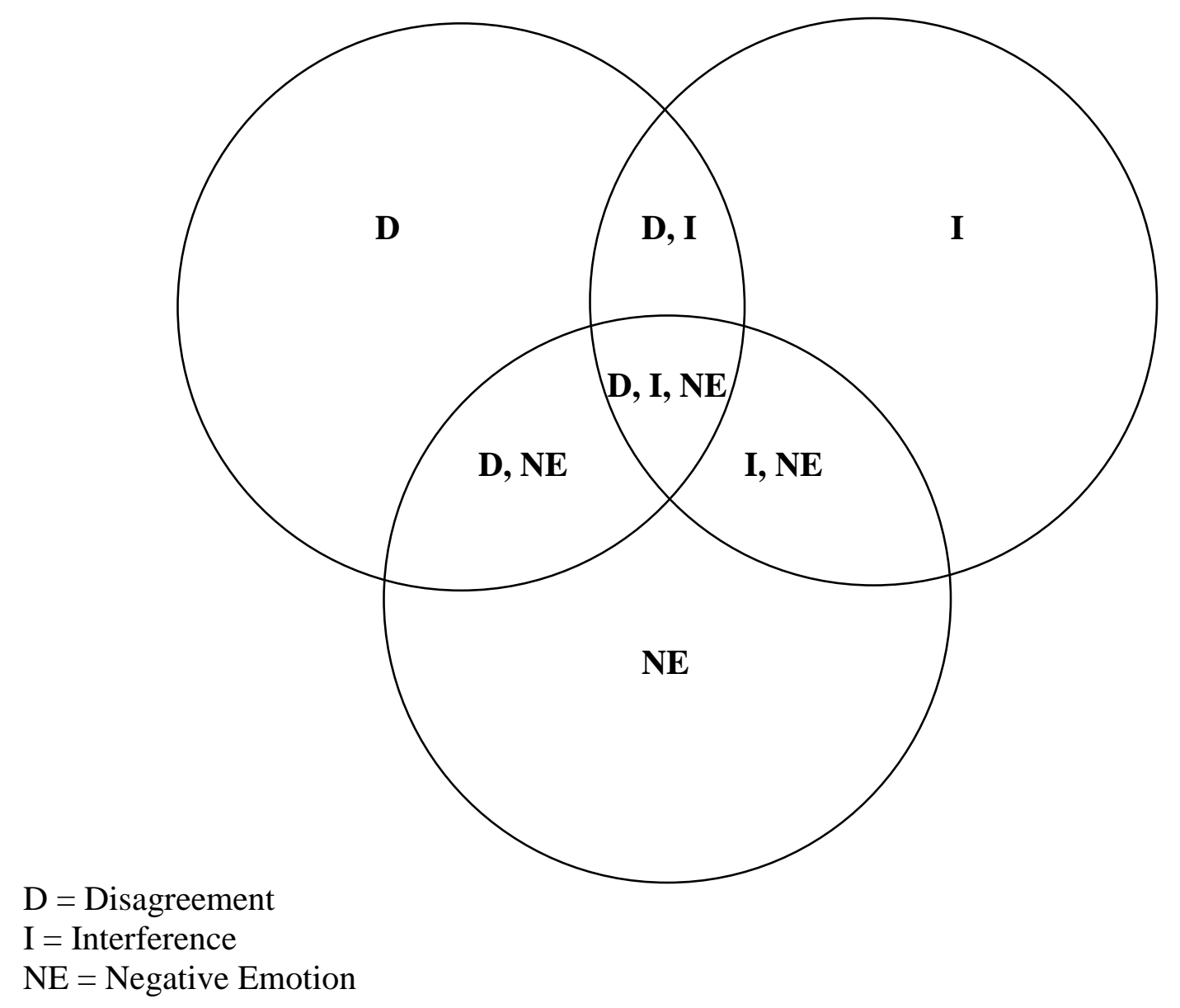


Figure 3: Transactional Model of Stress and Coping (Lazarus \& Folkman, 1984)

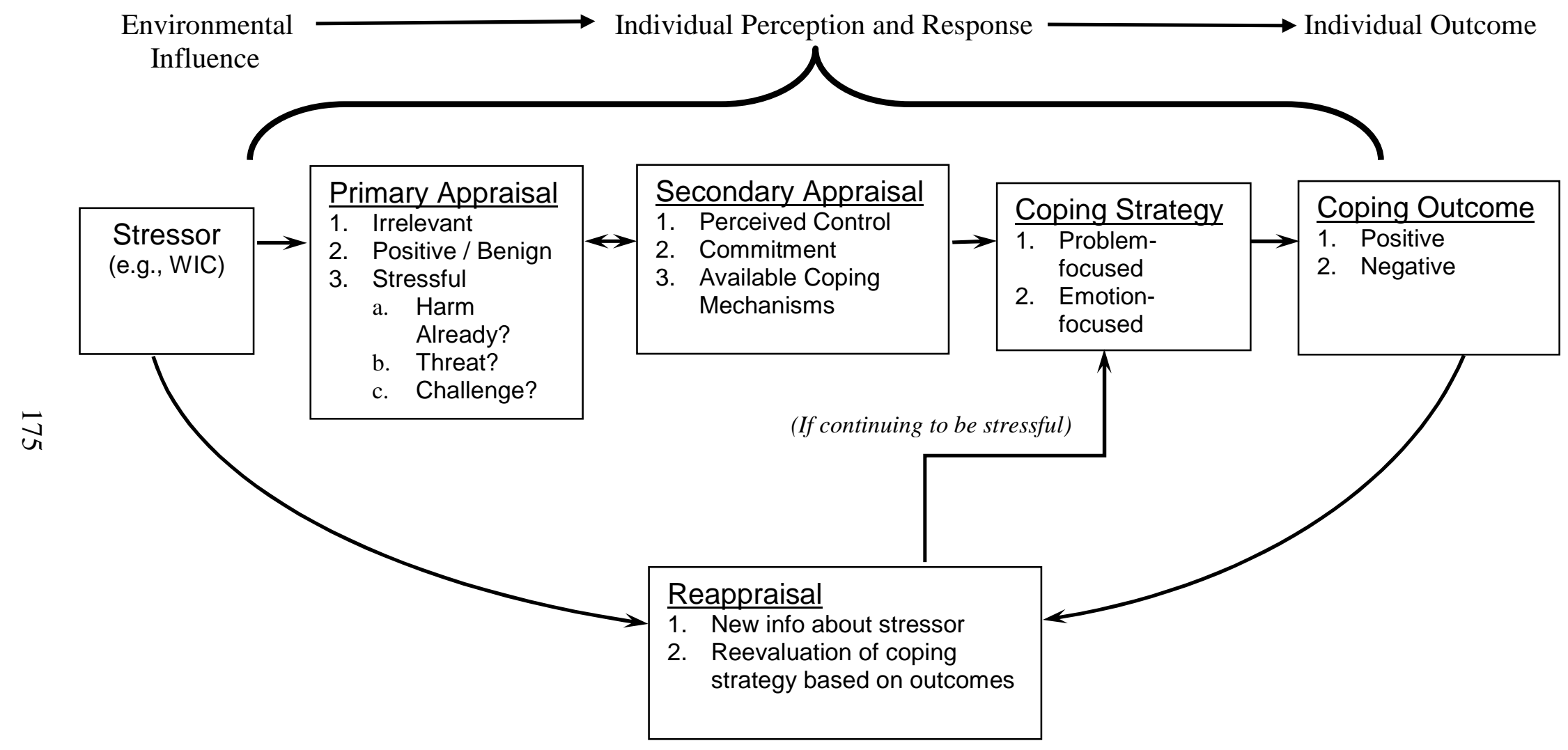




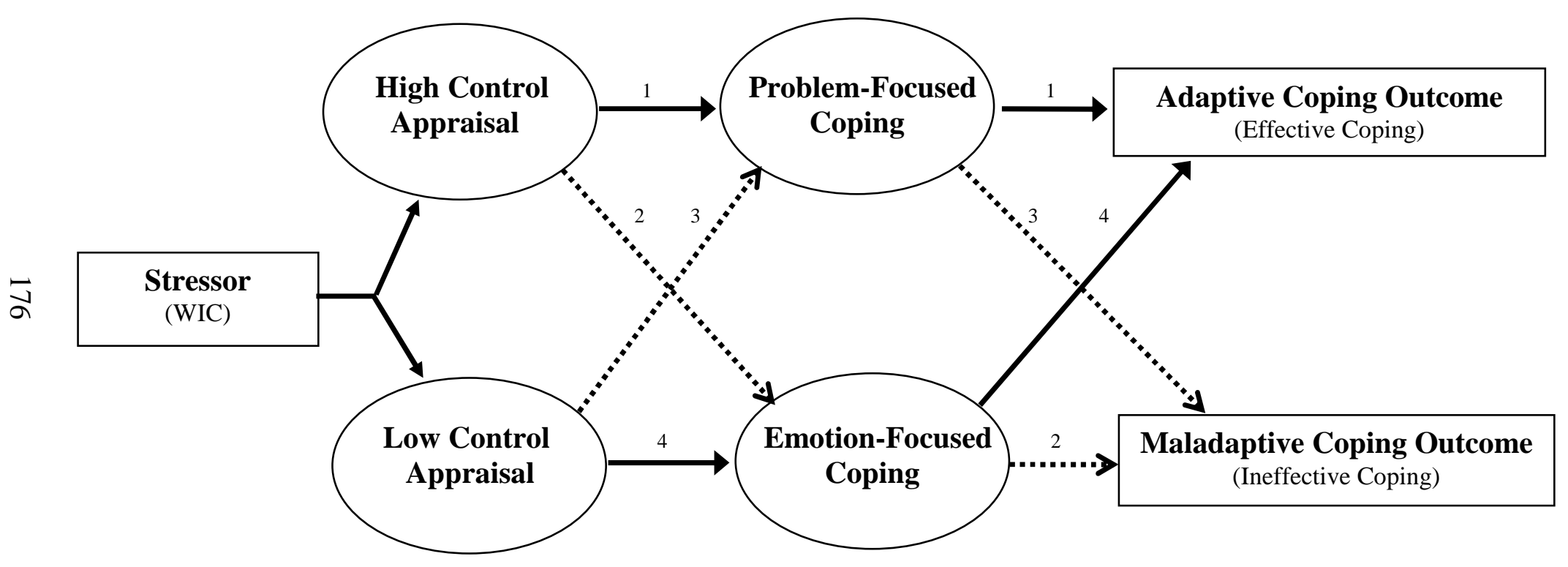

Note: Four pathways are specified in the above model, denoted by numbers. Solid lines indicate associations predicted to be effective or adaptive; dotted lines represent predicted ineffective relationships. 
Figure 5: The Goodness of Fit Hypothesis Testing Model

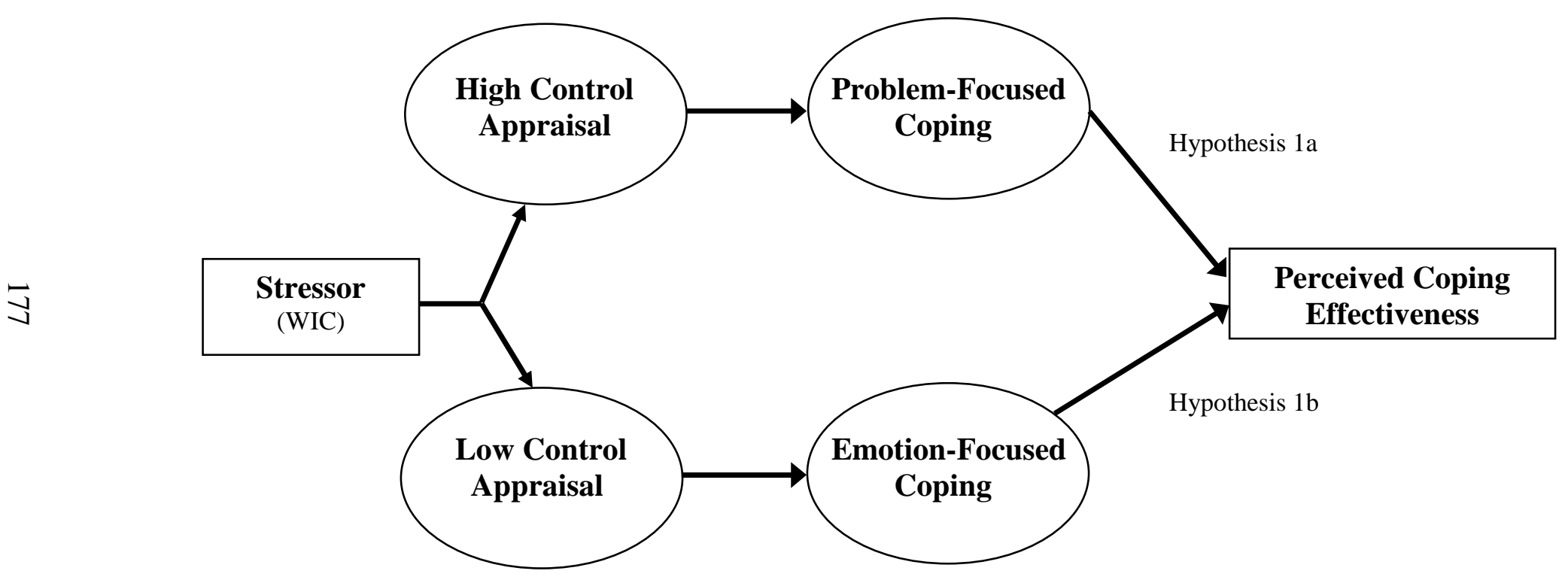

Hypothesis 1a: I predict that problem-focused coping will be rated as more effective on weeks when perceived control is higher versus weeks when perceived control is lower.

Hypothesis 1b: I predict that emotion-focused coping will be rated as more effective on weeks when perceived control is lower versus weeks when perceived control is higher. 
Figure 6: The Goodness of fit Hypothesis Testing Model Moderated by Occupational Tenure

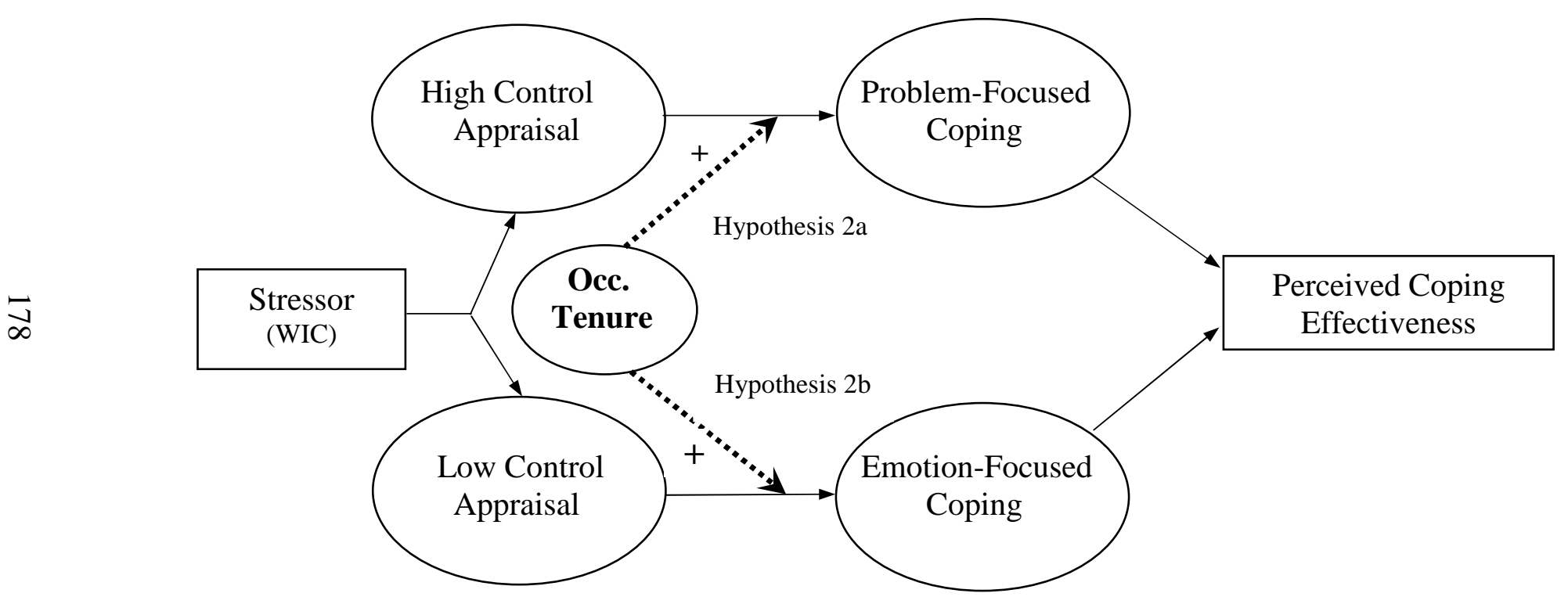

Note: Occupational tenure is added to the model as a moderating variable of the goodness of fit relationship between control appraisal and coping type - denoted by a bold dotted line.

Hypothesis 2a: The problem-focused coping-high perceived control relationship (i.e., Hypothesis 1a) will be stronger for nurses with greater occupational tenure relative to nurses with less occupational tenure.

Hypothesis 2b: The emotion-focused coping-low perceived control relationship (i.e., Hypothesis $1 b$ ) will be stronger for nurses with greater occupational tenure relative to nurses with less occupational tenure. 
Figure 7: Occupational Tenure Main Effects on Coping Frequency

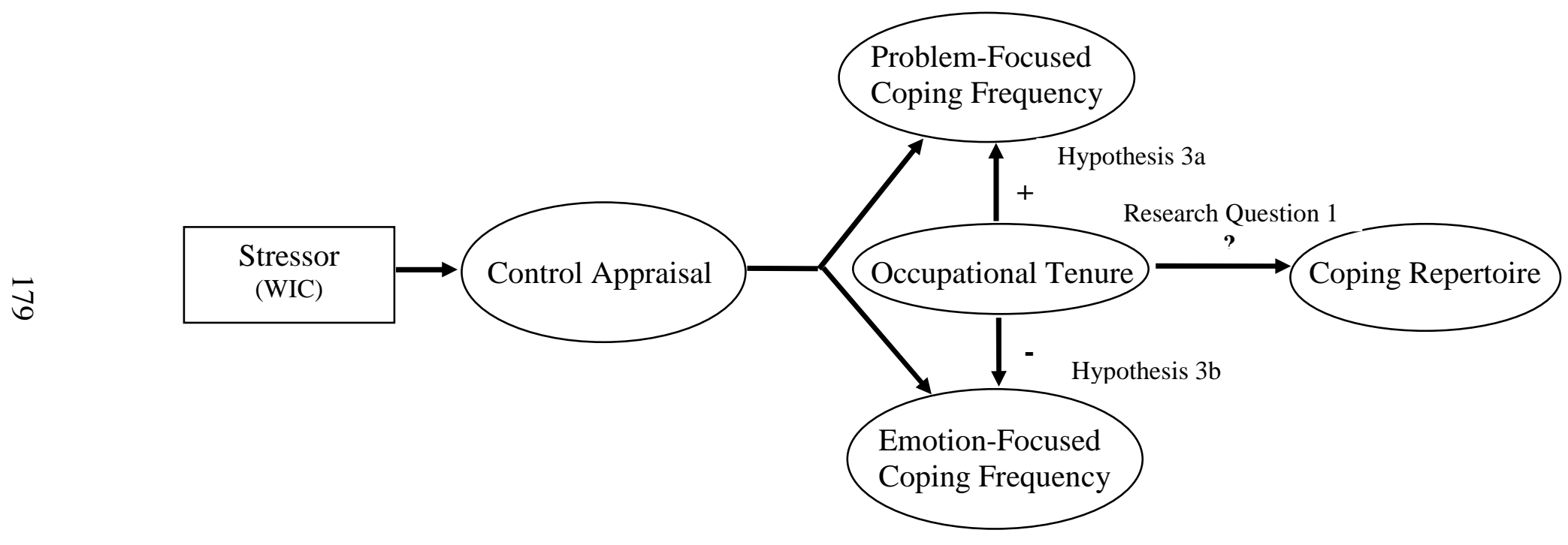

Hypothesis 3a: Nurses with greater occupational tenure will engage in more problem-focused coping across study days in response to workplace interpersonal conflicts compared to nurses with less occupational tenure.

Hypothesis 3b: Nurses with less occupational tenure will engage in more emotion-focused coping across study days in response to workplace interpersonal conflicts compared to nurses with greater occupational tenure.

Research Question 1: Is occupational tenure related to changes in coping frequency variance (i.e., coping frequency standard deviations)? 
Figure 8: The Oregon Nurse Retention Project Research Design

\section{ONRP Research Design}

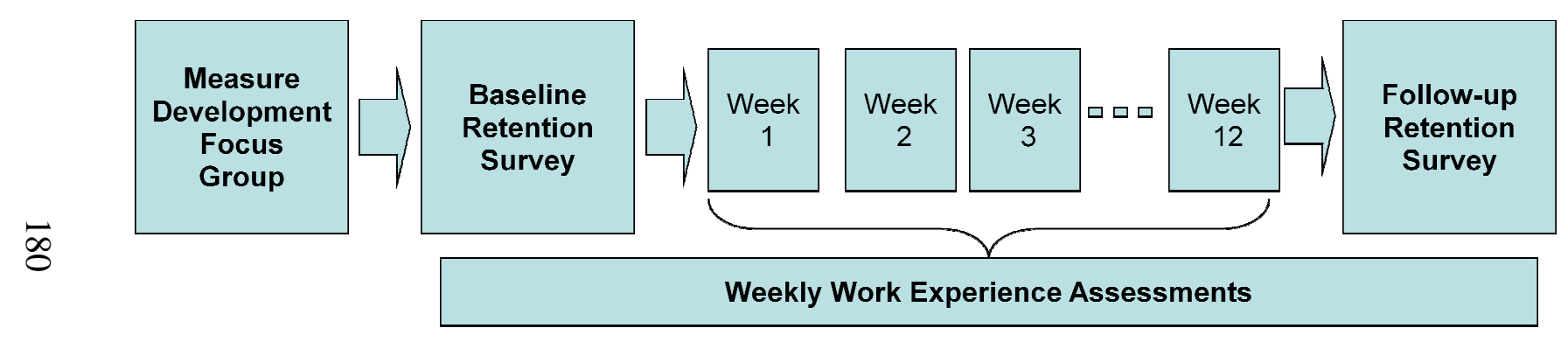




\section{References}

AbuAlRub, R. F. (2004). Job stress, job performance, and social support among hospital nurses. Journal of Nursing Scholarship, 36(1), 73-78.

AbuAlRub, R. F. (2006). Replication and examination of research data on job stress and coworker social support with internet and traditional samples. Journal of Nursing Scholarship, 38(2), 200-204.

Adip, S. M., Al-Shatti, A. K., Kamal, S., El-Gerges, N., \& Al-Raquem, M. (2002). Violence against nurses in healthcare facilities in Kuwait. International Journal of Nursing Studies, 39, 469-478.

Aiken L.H., Clarke S.P., Sloane D., Sochalski J. \& Silber J.H. (2002) Hospital nurse staffing and patient mortality, nurse burnout, and job dissatisfaction. Journal of the American Medical Association, 288(16), 1987-1993.

Aiken, L.S., \& West, S.G. (1991). Multiple regression: Testing and interpreting interactions. New York: Russell Sage.

Aldwin, C. M., \& Revenson, T. A. (1987). Does coping help? A reexamination of the relation between coping and mental health. Journal of Personality and Social Psychology, 53(2), 337-348.

Aldwin, C., Folkman, S., Shaefer, C., Coyne, J, \& Lazarus, R. (1980). Ways of Coping Checklist: A process measure. Paper presented at the 88th Annual Convention of the American Psychological Association, Montreal, Quebec, Canada.

Allison, P. D. (2002). Missing Data. Thousand Oaks, CA: Sage

Almost, J. (2006). Conflict within nursing work environments: Concept analysis. Journal of Advanced Nursing, 53(4), 444-453.

Andersson, L. M., \& Pearson, C. M. (1999). Tit for tat? The spiraling effect of incivility in the workplace. Academy of Management Review, 24, 452-471.

Appelberg, K., Romanov, K., Heikkila, K., Honkasalo, M., \& Koskenvuo, M. (1996). Interpersonal conflict as a predictor of work disability: A follow-up study of 15,348 Finnish employees. Journal of Psychosomatic Research, 40(2), 157-167.

Aspinwall, L.G., \& Taylor, S.E (1997). A stitch in time: Self-regulation and proactive coping. Psychological Bulletin, 121(3), 417-436.

Atkinson, M., \& Violato, C. (1994). Neuroticism and coping with anger: The transsituational consistency of coping responses. Personality and Individual Differences, 17(6), 769-782.

Baggs, J. G., \& Ryan, S. A. (1990). ICU nurse-physician collaboration and nursing satisfaction. Nursing Economics, 8(6), 386-392).

Bakker, A. B., \& Demerouti, E. (2007). The Job Demands-Resources model: State of the art. Journal of Managerial Psychology, 22, 309-328.

Bakker, A.B., Demerouti, E., De Boer, E. \& Schaufeli, W.B. (2003). Job demands and job resources as predictors of absence duration and frequency. Journal of Vocational Behavior, 62, 341-56. 
Bakker, A. B., Kilmer, C. H., Siegrist, J., \& Schaufeli, W. B. (2000). Effort-reward imbalance and burnout among nurses. Journal of Advanced Nursing, 31(4), 884891.

Ball, R. (2004). Workplace stress sucks $\$ 300$ billion annually from corporate profits. Customer Interaction Solutions, 23(5), 62-63.

Baltimore, J. J. (2006). Nurse collegiality: Fact or fiction. Nursing Management, 37(5), 28-36.

Barki, H., \& Hartwick, J. (2001). Interpersonal conflict and its management in information systems development. MIS Quarterly, 25, 217-250.

Barki, H., \& Hartwick, J. (2004). Conceptualizing the construct of interpersonal conflict. The International Journal of Conflict Management, 15(3), 216-244.

Barling, J., Dupre, K. E., \& Kelloway, E. K. (2009). Predicting workplace aggression and violence. Annual Review of Psychology, 60, 671-692.

Baum, A., Fleming, R., \& Singer, J. E. (1983). Coping with victimization by technological disaster. Journal of Social Issues, 39(2), 117-138.

Baumeister, R. F., Bratslavsky, E., Muraven, M., \& Tice, D. M. (1998). Ego-depletion: Is the active self a limited resource? Journal of Personality and Social Psychology, 74, 1252-1265.

Beach, S. R. H., Martin, J. K., Blum, T. C., \& Roman, P. M. (1993). Effects of marital and co-worker relationships on negative affect: Testing the central role of marriage. The American Journal of Family Therapy, 21(4), 313-323.

Bennett, J. B., Cook, R. F., \& Pelletier, K. R. (2003). Toward an integrated framework for comprehensive organizational wellness: Concepts, practices, and research in workplace health promotion. In J. C. Quick \& L. E. Tetrick (Eds.), Handbook of occupational health psychology (pp. 69-96). Washington, D.C.: APA.

Bennett, R. J., \& Robinson, S. L. (2000). Development of a measure of workplace deviance. Journal of Applied Psychology, 85, 349-360.

Benyamini, Y., Gefen-Bardarian, Y., Gozlan, M., Tabiv, G., Shiloh, S., \& Kokia, E. (2008). Coping specificity: The case of women coping with infertility treatments. Psychology and Health, 23(2), 221-241.

Bergmann, T.J. \& Volkema, R.J. (1989). Understanding and managing interpersonal conflict at work: Its issues, interactive processes and consequences. In M.A. Rahim (Ed.), Managing conflict: An interdisciplinary approach (pp. 7-19). New York: Praeger.

Bergmann, T.J., \& Volkema, R.J. (1994). Issues, behavioral responses and consequences in interpersonal conflicts. Journal of Organizational Behavior, 15, 467-471.

Berkowitz, L. (1988). Frustrations, appraisals, and aversively stimulated aggression. Aggressive Behavior, 14, 3-11.

Bianchi, E. R. F. B. (2004). Stress and coping among cardiovascular nurses: A survey in Brazil. Issues in Mental Health Nursing, 25, 737-745.

Billeter-Koponen, S. \& Freden, L. (2005). Long-term stress, burnout and patient-nurse relations: Qualitative interview study about nurses' experiences. Scandinavian Journal of Caring Sciences, 19, 20-27. 
Boey, K. W. (1999). Distressed and stress resistant nurses. Issues in Mental Health Nursing, 19, 33-54.

Blake, R.R., \& Mouton, J.S. (1964). The managerial grid. Houston: Gulf Publishing.

Bolger, N. (1990). Coping as a personality process: A prospective study. Journal of Personality and Social Psychology, 59(3), 525-537.

Bolger, N., DeLongis, A., Kessler, R. C., \& Schilling, E. A. (1989). Effects of daily stress on negative mood. Journal of Personality and Social Psychology, 57(5), 808-818.

Bollen, K. A., \& Liang, J. (1988). Some properties of Hoelter's CN. Sociological Methods \& Research, 16(4), 492-503.

Bollen, K., \& Lennox, R. (1991). Conventional wisdom on measurement: A structural equation perspective. Psychological Bulletin, 110(2), 305-314.

Bradley, G. (2007). Job tenure as a moderator of stressor-strain relations: A comparison of experienced and new-start teachers. Work \& Stress, 21(1), 48-64.

Bruk-Lee, V., \& Spector, P. E. (2006) The social stressors-counterproductive work behaviors link: Are conflicts with supervisors and coworkers the same? Journal of Occupational Health Psychology, 11(2), 145-156.

Bryk, A. S., \& Raudenbush, S. W. (1992). Hierarchical linear models: Applications and data analysis methods. Newbury Park, CA: Sage.

Carver, C. S. (1997). You want to measure coping but your protocol's too long: Consider the Brief COPE. International Journal of Behavioral Medicine, 4(1), 92-100.

Carver, C. S., Pozo, C., Harris, S. D., Noriega, V., Scheier, M. F., Robinson, D. S., Ketcham, A. S., Moffat, F. L. Jr., \& Clark, K. C. (1993). How coping mediates the effect of optimism on distress: A study of women with early stage breast cancer. Journal of Personality and Social Psychology, 65(2), 375-390.

Carver, C. S., Scheier, M. F., \& Weintraub, J. K. (1989). Assessing coping strategies: A theoretically based approach. Journal of Personality and Social Psychology, 56(2), 267-283.

Chang, E. M., Hancock, K. M., Johnson, A., Daly, J., \& Jackson, D. (2005). Role stress in nurses: Review of related factors and strategies for moving forward. Nursing and Health Sciences, 7, 57-65.

Cheuk, W. H., Wong, K. S., Swearse, B., \& Rosen, S. (1997). Stress preparation, coping style, and nurses' experience of being spurned by patients. Journal of Social Behavior and Personality, 12(4), 1055-1064.

Clark, M. S., \& Grote, N. K. (1998). Why aren't indices of relationship costs always negatively related to indices of relationship quality? Personality and Social Psychology Review, 2, 2-17.

Cohen, F. (1987). Measurement of coping. In S. Y Kasl \& C. L. Cooper (Eds.), Stress and health: Issues in research methodology, (pp. 283-305). New York: Wiley.

Cohen, S., Miller, G. E., \& Rabin, B. S. (2001). Psychological stress and antibody response to immunization: A critical review of human literature. Psychosomatic Medicine, 63, 7-18. 
Compas, B. E., Banez, G. A., Malcarne, V., \& Worsham, N. (1991). Perceived control and coping with stress: A developmental perspective. Journal of Social Issues, 47(4), 23-34.

Compas, B. E., Malcarne, V., \& Fondacaro, K. M. (1988). Coping with stressful events in older children and young adolescents. Journal of Consulting and Clinical Psychology, 56(3), 405-411.

Colligan, T. W., \& Higgins, E. M. (2005). Workplace stress: Etiology and consequences. Journal of Workplace Behavioral Health, 21(2), 89-97.

Conger, J. J. (1956). Reinforcement theory and the dynamics of alcoholism. Quarterly Journal of Studies on Alcohol, 17, 296-305.

Conway, V. J., \& Terry, D. J. (1992). Appraised controllability as a moderator of the effectiveness of different coping strategies: A test of the goodness of fit hypothesis. Australian Journal of Psychology, 44, 1-7.

Cortina, L. M., Magley, V. J., Williams, J. H., \& Langhout, R. D. (2001). Incivility in the workplace: Incidence and impact. Journal of Occupational Health Psychology, 6(1), 64-80.

Cortina, L. M., \& Wasti, S. A. (2005). Profiles in coping: Responses to sexual harassment across persons, organizations, and cultures. Journal of Applied Psychology, 90(1), 182-192.

Cosier, R.A., Dalton, D.R. \& Taylor, L.A. (1991). Positive effects of cognitive conflict and employee voice. Employee Responsibilities and Rights Journal, 4, 7-11.

Costa, P.T. Jr., Somerfield, M. R., \& McCrae, R.R. (1996). Personality and coping: A reconceptualization. In M. Zeidner, \& S. Norman (Eds.), Handbook of coping: Theory, research, applications. (pp. 44-61). Oxford, England: John Wiley \& Sons.

Cox, S., Pakenham, K. I., \& Cole, S. (2010). A test of the Job Demands-Resources Model with HIV/AIDS volunteers. Journal of HIV/AIDS \& Social Services, 9(4), 332355.

Cutrona, C. E., \& Russell, D. (1990). Type of social support and specific stress: Toward a theory of optimal matching. In I. G. Sarason,\& B. R. Pierce (Eds.), Social support: An interactional view (pp. 219-366). New York, NY: Wiley.

Dana, D. (1999). Measuring the financial cost of organizational conflict. San Diego, CA: MTI Publications.

Davison, M.L., Kwak, N., Seo, Y.S., \& Choi, J. (2002). Using hierarchical linear models to examine moderator effects: Person-by-organization interactions. Organizational Research Methods, 5(3), 231-254.

de Carteret, J.C. (1994). Occupational stress claims: Effects on worker's compensation. American Association of Occupational Health Nurses Journal, 42, 484-498.

Decker, P. J., \& Borgen, F. H. (1993). Dimensions of work appraisal: Stress, strain, coping, job satisfaction, and negative affectivity. Journal of Counseling Psychology, 40(4), 470-478. 
de Dreu, C. K. W., \& Beersma, B. (2005). Conflict in organizations: Beyond effectiveness and performance. European Journal of Work and Organizational Psychology, 14, 105-117.

de Lange, A. H., Taris, T. W., Kompier, M. A. J., Houtman, I. L. D., \& Bongers, P. M. (2003). "The Very Best of the Millennium": Longitudinal Research and the Demand-Control-(Support) Model. Journal of Occupational Health Psychology, 8(4), 282-305.

DeLongis, A., \& Holtzman, S. (2005). Coping in context: The role of stress, social support, and personality in coping. Journal of Personality, 73(6), 1633-1652.

de Raeve, L., Jansen, N.W.H., van den Brandt, P.A., Vasse, R., \& Kant, I.J. (2009). Interpersonal conflicts at work as a predictor of self-reported health outcomes and occupational mobility. Occupational \& Environmental Medicine, 66(1), 16-22.

Deutsch, M. (1949). A theory of cooperation and competition. Human Relations, 2, 129152.

Deutsch, M. (1962). Cooperation and trust: Some theoretical notes. In M. R. Jones (Ed.) Nebraska Symposium on Motivation. Lincoln: University of Nebraska Press.

Deutsch, M. (1973). The resolution of conflict, New Haven, CT: Yale University Press.

Deutsch, M. (1990). Sixty years of conflict. International Journal of Conflict Management, 1, 237-263.

Dewe, P. J. (1987). Identifying the causes of nurses' stress: A survey of New Zealand nurses. Work \& Stress, 1(1), 15-24.

Dewe, P. J. (1989). Examining the nature of work stress: Individual evaluations of stressful experiences and coping. Human Relations, 42(11), 993-1013.

Dewe, P. (1993). Coping and the intensity of nursing stressors. Journal of Community \& Applied Social Psychology, 3, 299-311.

Diaz, A. L., \& McMillin, J. D. (1991). A definition and description of nurse abuse. Western Journal of Nursing Research, 13(1), 97-109.

Dollard, J., Doob, L., Miller, N., Mowrer, O., \& Sears, R. (1939). Frustration and aggression. New Haven, CN: Yale University Press.

Drumheller, P. M. Jr., Eicke, F. J., \& Scherer, R. F. (1991). Cognitive appraisal and coping of students varying in stress level during three stages of a college examination. Journal of Social Behavior and Personality, 6(2), 237-254.

Duddle, M., \& Boughton, M. (2007). Intraprofessional relations in nursing. Journal of Advanced Nursing, 59(1), 29-37.

Dunn, H. (2003). Horizontal violence among nurses in the operating room. AORN Journal, 78(6), 977-988.

Eisenberger, R., Huntington, R., Hutchison, S. \& Sowa, D. (1986). Perceived organizational support. Journal of Applied Psychology, 71(3), 500-507.

Elfering, A., Semmer, N. K., \& Grebner, S. (2006). Work stress and patient safety: Observer-rated work stressors as predictors of characteristics of safety-related events reported by young nurses. Ergonomics, 49(5-6), 457-469. 
Enders, C.K. \& Tofighi, D. (2007). Centering predictor variables in cross-sectional multilevel models: A new look at an old issue. Psychological Methods, 12, 121138.

Endler, N. S., \& Parker, J. D. A. (1990). Multidimensional assessment of coping: A critical evaluation. Journal of Personality and Social Psychology, 58(5), 844-854.

Farrell, G. A. (1999). Aggression in clinical settings: Nurses' views - a follow-up study. Journal of Advanced Nursing, 29(3), 532-541.

Farrell, G. A. (2001). From tall poppies to squashed weeds: Why don't nurses pull together more? Journal of Advanced Nursing, 35(1), 26-33.

Felton, B. J., \& Revenson, T. A. (1984). Coping with chronic illness: a study of illness controllability and the influence of coping strategies on psychological adjustment. Journal of Consulting and Clinical Psychology, 52, 343-353

Festinger, L. (1954). A theory of social comparison processes. Human Relations, 7, 117140.

Florian, V., Mikulincer, M., \& Taubman, O. (1995). Does hardiness contribute to mental health during a stressful real-life situation? The roles of appraisal and coping. Journal of Personality and Social Psychology, 68(4), 687-695.

Florio, G. A., Donnelly, J. P., \& Zevon, M. A. (1998). The structure of work-related stress and coping among oncology nurses in high-stress medical settings: A transactional analysis. Journal of Occupational Health Psychology, 3(3), 227-242.

Folkman, S., \& Lazarus, R. S. (1980). An analysis of coping in a middle-aged community sample. Journal of Health and Social Behavior, 21, 219-239

Folkman, S. \& Lazarus, R. S. (1981). Reply to Shinn and Krantz. Journal of Health and Social Behavior, 22(4), 457-459.

Folkman, S. \& Lazarus, R. S. (1985). If it changes it must be a process: A study of emotion and coping during three stages of a college examination. Journal of Personality and Social Psychology, 48, 150-170.

Folkman, S. \& Lazarus, R. S. (1988). Coping as a mediator of emotion. Journal of Personality and Social Psychology, 54(3), 466-475.

Folkman, S., Lazarus, R.S., Gruen, R.J., \& DeLongis, A. (1986). Appraisal, coping, health status, and psychological symptoms. Journal of Personality and Social Psychology, 50(3), 571-579.

Forsythe, C. J., \& Compas, B. E. (1987). Interaction of cognitive appraisals of stressful events and coping: Testing the goodness of fit hypothesis. Cognitive Therapy and Research, 11, 473-485.

Fox, M. L., Dwyer, D. J., \& Ganster, D. C. (1993). Effects of stressful job demands and control on physiological and attitudinal outcomes in a hospital setting. The Academy of Management Journal, 36(2), 289-318.

Frone, M. R. (2000). Interpersonal conflict at work and psychological outcomes: Testing a model among young workers. Journal of Occupational Health Psychology, 5(2), 246-255. 
Frone, M. R. (2008). Are work stressors related to employee substance use? The importance of temporal context in assessments of alcohol and illicit drug use. Journal of Applied Psychology, 93(1), 199-206.

Frydenberg, E., \& Lewis, R. (1994). Coping with different concerns: Consistency and variation in coping strategies used by adolescents. Australian Psychologist, 29(1), 45-48.

Ganster, D. C. (1989). Worker control and well-being: A review of research in the workplace. In S. Sauter, J. Hurrell, and C. Cooper (Eds.), Job Control and Worker Health (pp. 3-24), John Wiley and Sons, Chichester.

Goldstein, A. P. (1994). The ecology of aggression. New York: Plenum.

Gonzalez-Morales, M. G., Peiro, J. M., Rodriguez, I., \& Greenglass, E. R. (2006). Coping and distress in organizations: The role of gender in work stress. International Journal of Stress Management, 13(2), 228-248.

Gotay, C. C., Crockett, S., \& West, C. (1985). Palliative home care nursing: Nurses' perceptions of roles and stress. Canada's Mental Health, 33(2), 6-9.

Greeley, J., \& Oei, T. (1999). Alcohol and tension reduction. In K. E. Leonard \& H. T. Blane (Eds.), Psychological theories of drinking and alcoholism, 2nd ed., (pp. 1453). New York: Guilford Press.

Haan, N. (1992). The assessment of coping, defense, and stress. In L. Goldberger \& S. Breznitz (Eds.), Handbook of stress: Theoretical and clinical aspects $\left(2^{\text {nd }}\right.$ ed., pp. 258-273). New York: Free Press.

Hackett, R. D., \& Bycio, P. (1996). An evaluation of employee absenteeism as a coping mechanism among hospital nurses. Journal of Occupational \& Organizational Psychology, 69(4), 327-328.

Hahn, S. (2000). The effects of locus of control on daily exposure, coping and reactivity to work interpersonal stressors: A diary study. Personality and Individual Differences, 29, 729-748.

Hamburg, D. A. (1974). Coping behavior in life-threatening circumstances. Psychotherapy and Psychoanalysis, 20, 308-321.

Hardy, G. E., Woods, D., \& Wall, T. D. (2003). The impact of psychological distress on absence from work. Journal of Applied Psychology, 88, 306-314.

Harvey, S., Blouin, C., \& Stout, D. (2006). Proactive personality as a moderator of outcomes for young workers experiencing conflict at work. Personality and Individual Differences, 40, 1063-1074.

Havens, D.S., Vasey, J., Gittell, J.H., \& Lin W.T. (2010). Relational coordination among nurses and other providers: Impact on the quality of patient care. Journal of Nursing Management, 18, 926-937.

Havlovic, S. J. \& Keenan, J. P. (1995). Coping with work stress: The influence of individual differences. In P. Perrewe (Ed.), Occupational stress: A handbook (pp. 179-192). Philadelphia, PA: Taylor \& Francis.

Henderson, A. S., \& Bostock, F. T. (1975). Coping behavior: Correlates of survival on a raft. Australian and New Zealand Journal of Psychiatry, 9, 221-223. 
Hepburn, C. G., Loughlin, C. A., \& Barling, J. (1997). Coping with chronic work stress. In B.H. Gottlieb (Ed.), Coping with chronic stress (pp. 343-366). New York: Plenum.

Hershcovis, M. S., \& Barling, J. (2006). Preventing insider-initiated workplace violence.In E. K. Kelloway, J. Barling, \& J. J. Hurrell, Jr. Handbook of workplace violence (pp. 607-632). Thousand Oaks, CA: Sage Publications, Inc.

Hobfoll, S.E. (1989). Conservation of resources: A new attempt at conceptualizing stress. American Psychologist, 44(3), 513-524.

Hobfoll, S. E., \& Lilly, R. S. (1993). Resource conservation as a strategy for community psychology. Journal of Community Psychology, 21, 128-148.

Holtzman, S., Newth, S., \& DeLongis, A. (2004). The role of social support in coping with daily pain among patients with Rheumatoid Arthritis. Journal of Health Psychology, 9, 677-695.

Hox, J. (2002). Multilevel Analysis; Techniques and Applications. Mahwah, New Jersey.

Hu, L., \& Bentler, P.M. (1999). Cutoff criteria for fit indexes in covariance structure analysis: Conventional criteria versus new alternatives. Structural Equation Modeling, 6(1), 1-55.

Huey, F. L., \& Hartley, S. (1988). What keeps nurses in nursing: 3,500 nurses tell their stories. The American Journal of Nursing, 88(2), 181-188.

Hughes, T. L., Howard, M. J., \& Henry, D. (2002). Nurses' use of alcohol and other drugs: Findings from a national probability sample. Substance Use \& Misuse, 37(11), 1423-1440.

Hyman, S. M. \& Sinha, R. (2009). Stress-related factors in cannabis use and misuse: Implications for prevention and treatment. Journal of Substance Abuse Treatment, 36(4), 400-413.

Inoue, A. \& Kawakami, N. (2010). Interpersonal conflict and depression among Japanese workers with high or low socioeconomic status: Findings from the Japan Work Stress and Health Cohort Study. Social Science \& Medicine, 71, 173-180.

Jehn, K. A. (1995). A multimethod examination of the benefits and detriments of intragroup conflict. Administrative Science Quarterly, 40, 256-282.

Jehn, K. A. (1997). A qualitative analysis of conflict types and dimensions in organizational groups. Administrative Science Quarterly, 42, 530-557.

Jensen-Campbell, L. A. \& Graziano, W. G. (2000). Beyond the school yard: Relationships as moderators of daily interpersonal conflict. Personality and Social Psychology Bulletin, 26, 923-935.

Johnston, V. (2007). Biological pathways between occupational stress and work-related musculoskeletal disorders of the neck and upper extremity. Physical Therapy, 12, 335-345.

Johnston, D.W., Beedie, A., \& Jones, M.C. (2006). Using computerized ambulatory diaries for the assessment of job characteristics and work-related stress in nurses. Work \& Stress, 20(2), 163-172.

Jorgensen, R.S., Dusek, J.B., Richards, C.S., \& McIntyre, J.G. (2009). An experimental investigation of consistency in female undergraduates' reports of coping efforts 
for the same versus different stressful situations. Canadian Journal of Behavioural Science, 41(1), 51-54.

Judd, C.M. \& Kenny, D.A. (1981). New York, NY: Cambridge University Press.

Karasek, R. A. (1979). Job demands, job decision latitude, and mental strain: Implications for job redesign. Administrative Science Quarterly, 24(2), 285-308.

Karasek, R., \& Theorell, T. (1990). Healthy work: Stress,productivity, and the reconstruction of working life. New York: Basic Books.

Katz, D., \& Kahn, R. L. (1978). The Social Psychology of Organizations. New York: Wiley.

Keenan, A., \& Newton, T. J. (1985). Stressful events, stressors, and psychological strains in young professional engineers. Journal of Occupational Behavior, 6, 151-156.

Kelley, H. H., \& Thibaut, J. W. (1978). Interpersonal relations: A theory of interdependence. New York: Wiley.

Kelloway, E. K., Sivanathan, N., Francis, L., \& Barling, J. (2005). Poor leadership. In J. Barling, E. K. Kelloway, \& M. R. Frone (Eds.), Handbook of work stress (pp. 89112). Thousand Oaks, CA: SAGE Publications.

Kendall, E. \& Terry, D. J. (2008). Understanding adjustment following traumatic brain injury: Is the goodness of fit coping hypothesis useful? Social Science \& Medicine, 67, 1217-1224.

Kenny, D.A., Mohr, C.D., \& Levesque, M.J. (2001). A social relations variance partitioning of dyadic behavior. Psychological Bulletin, 127(1), 128-141.

Kivimaki, M., Virtanen, M., Elovainio, M., Kouvonen, A., Vaananen, A., \& Vahtera, J. (2006). Work stress in the etiology of coronary heart disease-a meta-analysis. Scandinavian Journal of Work Environment \& Health, 32(6), 431-442.

Kline, R.B. (1998). Principles and practice of structural equation modeling. New York: Guilford.

Knight, D. K. (2002). Customer orientation, self-monitoring, role stress, and job performance of contact employees: Implications for retailers. Unpublished dissertation, Texas Woman's University.

Kohler, J. M., Munz, D. C., \& Grawitch, M. J. (2006). Test of a dynamic stress model for organizational change: Do males and females require different models? Applied Psychology: An International Review, 55(2), 168-191.

Kohn, P. M. (1996). On coping adaptively with daily hassles. In M. Zeidner, \& S. Norman (Eds.), Handbook of coping: Theory, research, applications. (pp. 181201). Oxford, England: John Wiley \& Sons.

Krajewski, H. T., \& Goffin, R. D. (2005). Predicting occupational coping responses: The interactive effect of gender and work stressor context. Journal of Occupational Health Psychology, 10(1), 44-53.

Kreft, I., de Leeuw, J., \& Aiken, L. S. (1995). The effects of different forms of centering in hierarchical linear models. Multivariate Behavioral Research, 30(1), 1-21.

Lambert, V. A., Lambert, C. E., \& Ito, M. (2004). Workplace stressors, ways of coping and demographic characteristics as predictors of physical and mental health of Japanese hospital nurses. International Journal of Nursing Studies, 41, 85-97. 
Lambert, V. A., Lambert, C. E., \& Yamase, H. (2003). Psychological hardiness, workplace stress and related stress reduction strategies. Nursing and Health Sciences, (5), 181-184.

Lamontagne, A. D., Keegel, T., Louie, A. M., Ostry, A., \& Landsbergis, P. A. (2007). A systemic review of the job-stress intervention evaluation literature, 1990-2005. International Journal of Occupational and Environmental Health, 13(3), 268280.

Latack, J. C. (1986). Coping with job stress: Meausres and future directions for scale development. Journal of Applied Psychology, 71(3), 377-385.

Lazarus, R. S. (1991). Psychological stress in the workplace. In P. Perrewe (Ed.), Handbook on job stress [Special issue]. Journal of Social Behavior and Personality, 6(7), 1-13.

Lazarus, R. S. (1993). Coping theory and research: Past, present and future. Psychosomatic Medicine, 55, 237-247.

Lazarus, R. S. (2001). Conservation of Resources Theory (COR): Little more than words masquerading as a new theory. Applied Psychology: An International Review, 50(3), 381-191.

Lazarus, R.S. (2006). Emotions and interpersonal relationships: Toward a personcentered conceptualization of emotions and coping. Journal of Personality, 74(1), 9-46.

Lazarus, R. S., Averill, J. R., \& Opton, E. M. (1974). The psychology of coping: Issues of research and assessment. In G. V. Coelho, D. A. Hamburg, \& J. E. Adams (Eds.) Coping and adaptation (pp. 287-327). New York: Basic Books.

Lazarus, R. S., DeLongis, A., Folkman, S., \& Gruen, R. (1985). Stress and adaptational outcomes: The problem of confounded measures. American Psychologist, 40(7), 770-779.

Lazarus, R. S., \& Folkman, S. (1984). Stress, appraisal, and coping. New York: Springer.

Lee, V. (2007). Measuring social stressors in organizations: The development of the Interpersonal Conflict in Organizations Scale (ICOS). Unpublished doctoral thesis, University of South Florida, Tampa.

Leiper, J. (2005). Nurse against nurse: How to stop horizontal violence. Nursing, 35(3), 44-45.

Leiter, M. P. (1991). Coping patterns as predictors of burnout: The function of control and escapist coping patterns. Journal of Organizational Behaviour, 12, 123-144.

Leiter, M. P. (2005). Perception of risk: An organizational model of occupational risk, burnout, and physical symptoms. Anxiety, Stress, and Coping, 18(2), 131-144.

Leitner, K., \& Resch, M. (2005). Do the effects of job stressors on health persist over time? A longitudinal study with observational stressor measures. Journal of Occupational Health Psychology, 10(1), 18-30.

Little, R., \& Rubin, D. (1987). Statistical analysis with missing data. New York: John Wiley. 
Lu, L. (1996). Coping consistency and emotional outcome: Intra-individual and interindividual analyses. Personality and Individual Differences, 21(4), 583-589.

Lu, L., Kao, S. F., Siu, Q. L., \& Lu, C. Q. (2010). Work stressors, Chinese coping strategies, and job performance in Greater China. International Journal of Psychology, 45(4), 294-302.

Ma, Z., Lee, Y., Yu, K.H. (2008). Ten years of conflict management studies: Themes, concepts and relationships. International Journal of Conflict Management, 19(3), 234-248.

Macik-Frey, M., Quick, J. C., \& Nelson, D. L. (2007). Advances in occupational health: From a stressful beginning to a positive future. Journal of Management, 33, 809840

Marco, C. A., Neale, J. M., Schwartz, J. E., Shiffman, S., \& Stone, A. A. (1999). Coping with daily events and short-term mood changes: An unexpected failure to observe effects of coping. Journal of Consulting and Clinical Psychology, 67(5), 755-764.

Maruyama, G.M. (1998). Basics of structural equation modeling. Thousand Oaks, CA: Sage.

Masel, C. N., Terry, D. J., \& Gribble, M. (1996). The effects of coping on adjustment: Re-examining the goodness of fit model of coping effectiveness. Anxiety, Stress, and Coping, 9, 279-300.

Matteson, M.T., \& Ivancevich, J.M. (1987). Controlling work stress. London, JosseyBass.

McMahan, E. M., Hoffman, K., \& McGee, G. W. (1994). Physician-nurse relationships in clinical settings: A review and critique of the literature, 1966-1992. Medical Care Review, 51(1), 83-112.

Meijman, T. F., \& Mulder, G. (1998). Psychological aspects of workload. In P. J. D. Drenth \& H. Thierry (Eds.), Handbook of work and organizational psychology, Vol. 2 Work psychology (pp. 5-33). Hove, England: Psychology Press.

Meissner, J.E. (1999). Nurses: Are we still eating our young? Nursing, 29(2), 42-44.

Mohr, C. D., Armeli, S., Ohannessian, C. M., Tennen, H., Carney, A., Affleck, G., \& Del Boca, F. K. (2003). Daily interpersonal experiences and distress: Are women more vulnerable? Journal of Social and Clinical Psychology, 22(4), 393-423.

Mohr, C. D., Armeli, S., Tennen, H., Carney, M. A., Affleck, G., \& Hromi, A. (2001). Daily interpersonal experiences, context, and alcohol consumption: Crying in your beer and toasting good times. Journal of Personality and Social Psychology, 81(3), 489-500.

Mohr, C.D., Armeli, S., Tennen, H., Temple, M., Todd, M., Clark, J., \& Carney, M.A. (2005). Moving beyond the keg party: A daily process study of college student drinking motivations. Psychology of Addictive Behaviors, 19(4), 392-403.

Morrill, C., \& Thomas, C. K. (1992). Organizational conflict management as disputing process. Human Communication Research, 18, 400-428.

Moser, K. \& Galais, N. (2007). Self-monitoring and job performance: The moderating role of tenure. International Journal of Selection and Assessment, 15(1), 83-93. 
Mulaik, S.A., James, L.R., van Alstine, J., Bennett, N., Lind, S., \& Stilwell, C.D. (1989). Evaluation of goodness-of-fit indices for structural equation models. Psychological Bulletin, 105(3), 430-445.

Muraven, M., \& Baumeister, R.F. (2000). Self-regulation and depletion of limited resources: Does self-control resemble a muscle? Psychological Bulletin, 126, 247259.

Neal, M.B., \& Hammer, L.B. (2007). Working couples caring for children and aging parents: Effects on work and well-being. Mahwah, NJ: Lawrence Erlbaum Associates, Inc.

Neal, M.B., \& Hammer, L.B. (2009). Dual-earner couples in the sandwiched generation: Effects of coping strategies over time. The Psychologist-Manager Journal, 12, 205-234.

Newsom, J. T., Nishishiba, M., Morgan, D. L., \& Rook, K. S. (2003). The relative importance of three domains of positive and negative exchanges: A longitudinal model with comparable measures. Psychology and Aging, 18(4), 746-754.

Nezlek, J. B., \& Plesko, R. M. (2003). Affect- and self-based models of relationships between daily events and daily well-being. Personality and Social Psychology Bulletin, 29(5), 584-596.

Nolan, K.A., Shope, C.B., Citrome, L., \& Volavka, J. (2009). Staff and patient views of the reasons for aggressive incidents: A prospective, incident-based study. Psychiatric Quarterly, 80, 167-172.

O'Brien, T. B. \& DeLongis, A. (1996). The interactional context of problem-, emotion-, and relationship-focused coping: The role of the big five personality factors. Journal of Personality, 64(4), 775-813.

Pandey, S., \& Srivastava, S. (2003). Work stress and coping as predictors of health status of career women. Journal of the Indian Academy of Applied Psychology, 29(1-2), 83-92.

Parasuraman, S., \& Hansen, D. (1987). Coping with work stressors in nursing: Effects of adaptive versus maladaptive strategies. Work and Occupations, 14, 88-105.

Park, C. L., Armeli, S., \& Tennen, H. (2004). Appraisal-coping goodness of fit: A daily internet study. Personality and Social Psychology Bulletin, 30, 558-569.

Park, C. L., Folkman, S., \& Bostrom, A. (2001). Appraisals of controllability and coping in caregivers and HIV+ men: Testing the goodness of fit hypothesis. Journal of Consulting and Clinical Psychology, 69, 481-488.

Parker, S. K., Axtell, C. M., \& Turner, N. (2001). Designing a safer workplace: Importance of job autonomy, communication quality, and supportive supervisors. Journal of Occupational Health Psychology, 6(3), 211-228.

Parker, J. D. A., Endler, N. S. (1996). Coping and defense: A historical review. In M. Zeidner, \& S. Norman (Eds.), Handbook of coping: Theory, research, applications. (pp. 3-23). Oxford, England: John Wiley \& Sons.

Pearlin, L.I. \& Schooler, C. (1978). The structure of coping. Journal of Health and Social Behavior, 19, 2-21. 
Pearson, C. M., Andersson, L. M., \& Wegner, J. W. (2001). When workers flout convention: A study of workplace incivility. Human Relations, 54: 1387-1419.

Pearson, C. M., \& Porath, C. L. (2005). On the nature, consequences and remedies of workplace incivility: No time for "nice"? Think again. Academy of Management Executive, 19(1), 7-18.

Peeters, M. C. W., Buunk, B. P., \& Schaufeli, W. B. (1995). A micro-analytic exploration of the cognitive appraisal of daily stressful events at work: The role of controllability. Anxiety, Stress, and Coping, 8, 127-139.

Pondy, L. R. (1967). Organizational conflict: Concepts and models. Administrative Science Quarterly, 12, 296-320.

Portello, J. Y. \& Long, B.C. (2001). Appraisals and coping with workplace interpersonal stress: A model for women managers. Journal of Counseling Psychology, 48(2), 144-156.

Porter, L. S., Marco, C. A., Schwartz, J. E., Neale, J. M., Shiffman, S., \& Stone, A. A. (2000). Gender differences in coping: A comparison of trait and momentary assessments. Journal of Social and Clinical Psychology, 19(4), 480-498.

Porter, L. S., \& Stone, A. A. (1996). On coping adaptively with daily hassles. In M. Zeidner, \& S. Norman (Eds.), Handbook of coping: Theory, research, applications. (pp. 133-150). Oxford, England: John Wiley \& Sons.

Preacher, K.J., Curran, P.J., \& Bauer, D.J. (2006). Computational tools for probing interaction effects in multiple linear regression, multilevel modeling, and latent curve analysis. Journal of Educational and Behavioral Statistics, 31, 437-448.

Pretrus, T., \& Kleiner, B. H. (2003). New developments concerning workplace safety training: Managing stress arising from work. Management Research News, 26(6), 68-76.

Pruitt, D.G. \& Rubin, J.Z. (1986). Social conflict: Escalation, stalemate, and settlement. New York: McGraw-Hill. Revised and updated as Pruitt, D.G., Rubin, J.Z. \& Kim, S.H. (1994). Social conflict: Escalation, stalemate, and settlement, 2nd ed. New York: McGraw-Hill.

Ptacek, J. T., Smith, R. E., \& Dodge, K. L. (2000). Gender differences in coping with stress: When stressor and appraisals do not differ. Personality and Social Psychology Bulletin, 20, 421-430.

Putnam, L. L., \& Poole, M. S. (1987). Conflict and negotiation. In F. M. Jablin, L. L. Putnam, K. H. Roberts, and L. W. Porter (Eds.), Handbook of Organizational Communication: An Interdisciplinary Perspective, (pp. 549-599). Newbury Park, CA: Sage.

Quick, J. C., Quick, J. D., Nelson, D. L., \& Hurrell, J. J. (1997). Preventative stress management in organizations. Washington, D.C.: APA.

Rahim, M.A. (1986). Referent role and styles of handling interpersonal conflict. Journal of Social Psychology, 126(1), 79-86.

Raudenbush, S. W. \& Bryk, A. S. (2002). Hierarchical linear models: Applications and data analysis methods, $2^{\text {nd }}$ edition. Newbury Park, CA: Sage. 
Raudenbush, S. W., Bryk, A. S., Cheong, Y. F., \& Congdon, R. T. (2010). HLM7. Hierarchical linear and nonlinear modeling. Lincolnwood, IL: Scientific Software International.

Rehm, J., Taylor, B., \& Room, R. (2006). Global burden of disease from alcohol, illicit drugs, and tobacco. Drug and Alcohol Review, 25, 503-513.

Richardson, K.M., \& Rothstein, H.R. (2008). Effects of occupational stress management intervention programs: A meta-analysis. Journal of Occupational Health Psychology, 13(1), 69-93.

Robinson, S., Murrells, T., \& Smith, E.M. (2005). Retaining the mental health nursing workforce: early indicators of retention and attrition. International Journal of Mental Health, 14, 230-242.

Roberts, S.J. (1983). Oppressed group behavior: Implications for nursing. Advances in Nursing Science, July, 21-30.

Roberts, S. M. (1995). Applicability of the goodness of fit hypothesis to coping with daily hassles. Psychological Reports, 77, 943-954.

Roesch, S. C., Aldridge, A. A., Stocking, S. N., Villodas, F., Leung, Q., Bartley, C. E., \& Black, L. J. (2010). Multilevel factor analysis of daily diary coping data: Modeling trait and state variation. Multivariate Behavioral Research, 45, 767789.

Romanov, K., Appelberg, K., Honkasalo, M., \& Koskenvuo, M. (1996). Recent interpersonal conflict at work and psychiatric morbidity: A prospective study of 15,530 employees aged 24-64. Journal of Psychosomatic Research, 40(2), 169176.

Rook, K. S., (2001). Emotional health and positive versus negative social exchanges: A daily diary analysis. Applied Developmental Science, 5(2), 86-97.

Rotondo, D. (1999). Individual-difference variables and career-related coping. The Journal of Social Psychology, 139(4), 458-471.

Ruehlman, L. S., \& Karoly, P. (1991). With a little flak from my friends: Development and preliminary validation of the test of negative social exchange (TENSE). Psychological Assessment, 3(1), 97-104.

Ruehlman, L. S., \& Wolchik, S. A. (1988). Personal goals and interpersonal support and hindrance as factors in psychological distress and well-being. Journal of Personality and Social Psychology, 55, 293-301.

Rydstedt, L. W., Devereux, J., \& Sverke, M. (2007). Comparing and combining the Demand-Control-Support Model and the Effort-Reward Imbalance Model to predict long-term mental strain. European Journal of Work and Organizational Psychology, 16(3), 261-278.

Salin, D. (2003). Ways of explaining workplace bullying: A review of enabling, motivating and precipitating structures and processes in the work environment. Human Relations, 56(10), 1213-1232.

Schat, A. C. H., Frone, M. R., \& Kelloway, E. K. (2006). Prevalence of workplace aggression in the U.S. workforce: Findings from a national study. In E. K. 
Kelloway, J. Barling, \& J. J. Hurrell, Jr. Handbook of workplace violence (pp. 47-89). Thousand Oaks, CA: Sage Publications, Inc.

Schieman, S., \& Reid, S. (2008). Job authority and interpersonal conflict in the workplace. Work and Occupations, 35(3), 296-326.

Schwartz, J.E., Neale, J., Marco, C., Shiffman, S.S., \& Stone, A.A. (1999). Does trait coping exist? A momentary assessment approach to the evaluation of traits. Journal of Personality and Social Psychology, 77(2), 360-369.

Schwartz, J. E., Pickering, T. G., Landsbergis, P. A. (1996). Work-related stress and blood pressure: Current theoretical models and considerations from a behavioral medicine perspective. Journal of Occupational Health Psychology, 1, 287-310.

Sherif, M., Harvey, O. J., White, B., Hood, W., \& Sherif, C. (1961). Intergroup conflict and cooperation: The robbers' cave experiment. Norman: Institute of Group Relations, University of Oklahoma.

Shinn, M., \& Krantz, D. H. (1981). Consistency in coping. Journal of Health and Social Behavior, 22(4), 456-457.

Shirom, A. (2003). Job-related burnout: A review. In J. C. Quick \& L. E. Tetrick (Eds.), Handbook of occupational health psychology (pp. 245-264). Washington, D.C.: APA.

Siegrist, J. (1996). Adverse health effects of high-effort/low reward conditions. Journal of Occupational Health Psychology, 1, 27-41.

Sinclair, R.R., C.P., Davidson, S., Sears, L.E., Deese, M.N., Wright, R.R., Waitsman, M., Jacobs, L., Cadiz, D. (2009). The Oregon Nurse Retention Project: Final Report to the Northwest Health Foundation. Unpublished Technical Report.

Skinner, E.A., Edge, K., Altman, J., \& Sherwood, H. (2003). Searching for the structure of coping: A review and critique of category systems for classifying ways of coping. Psychological Bulletin, 129(2), 216-269.

Skinner, E.A., \& Zimmer-Gembeck, M.J. (2007). The development of coping. Annual Review of Psychology, 58, 119-144.

Smith, C. S., \& Sulsky, L. (1995). An investigation of job-related coping strategies across multiple stressors and samples. In L. R. Murphy, J. J. Hurrell Jr., S. L. Sauter, \& G. P. Keita. Job stress interventions (pp. 109-123). Washington, DC: American Psychological Association.

Smith, M.J., Colligan, M.J., \& Hurrell, J.J., Jr (1977). A review of NIOSH psychological stress research. Paper presented at the UCLA Conference on Job Stress, Los Angeles, November.

Snijders, T. A. B., \& Bosker, R. J. (1999). Multilevel analysis: An introduction to basic and advanced multilevel modeling. London: Sage.

Sparrowe, R. T., \& Liden, R. C. (1997). Process and structure in leader-member exchange. Academy of Management Review, 22, 522-552.

Sparrowe, R. T., \& Liden, R. C. (2005). Two routes to influence: Integrating leadermember exchange and network perspectives. Administrative Science Quarterly, 50, 505-535. 
Spector, P. E., Fox, S., \& Domagalski, T. (2006). Emotions, violence and counterproductive work behavior. In E. K. Kelloway, J. Barling, \& J. J. Hurrell, Jr. Handbook of workplace violence (pp. 29-46). Thousand Oaks, CA: Sage Publications, Inc.

Spector, P. E., \& Jex, S. M. (1998). Development of four self-report measures of job stressors and strain: Interpersonal conflict at work scale, organizational constraints scale, quantitative workload inventory, and physical symptoms inventory. Journal of Occupational Health Psychology, 3(4), 356-367.

Spell, C. S., \& Arnold, T. (2007). An appraisal perspective of justice, structure, and job control as antecedents of psychological distress. Journal of Organizational Behavior, 28, 729-751.

Sternberg, R.J. \& Dobson, D.M. (1987). Resolving interpersonal conflicts: An analysis of stylistic consistency. Journal of Personality and Social Psychology, 52, 794-8 12.

Stone, A.A., \& Neale, J.M. (1984). New measure of daily coping: Development and preliminary results. Journal of Personality and Social Psychology, 46, 892-906.

Stone, A. A., Schwartz, J. E., Neale, J. M., Shiffman, S., Marco, C. A., Hickcox, M., Paty, J., Porter, L., Cruise, L. J. (1998). A comparison of coping assessed by ecological momentary assessment and retrospective recall. Journal of Personality and Social Psychology, 74, 1670-1680.

Sulsky, L., \& Smith, C.S. (2007). Work stress: Macro-level work stressors. In A. Monat, R.S. Lazarus, \& G. Reevy (Eds.) The Praeger Handbook on Stress and Coping, pp. 53-86.

Sundberg, L. L. (2003). The relationship between proactive coping skills and personal and organizational outcomes. Unpublished doctoral thesis, St. Ambrose University, Davenport, IO.

Tabak, N., \& Koprak, O. (2007). Relationship between how nurses resolve their conflicts with doctors, their stress and job satisfaction. Journal of Nursing Management, 15, 321-331.

Tajfel, H. \& Turner, J. C. (1986). The social identity theory of intergroup behavior. In S. Worchol \& W. G. Austin (Eds.), Psychology of Intergroup Relations (2nd ed). Chicago: Nelson-Hall.

Tennen, H., \& Affleck, G. (1996). Daily processes in coping with chronic pain: Methods and analytic strategies. In M. Zeidner, \& S. Norman (Eds.), Handbook of coping: Theory, research, applications. (pp. 151-180). Oxford, England: John Wiley \& Sons.

Tennen, H., Affleck, G., Armeli, S., \& Carney, A. (2000). A daily process approach to coping: Linking theory, research, and practice. American Psychologist, 55(6), 626-636.

Terry, D.J., \& Hynes, G.J. (1998). Adjustment to a low-control situation: Reexamining the role of coping responses. Journal of Personality and Social Psychology, 74(4), 1078-1092.

Tetrick, L.E., \& Quick, J.C. (2011). In J. C. Quick \& L. E. Tetrick (Eds.), Handbook of occupational health psychology (pp. 3-18). Washington, D.C.: APA. 
Theorell, T. (2003). To be able to exert control over one's own situation: a necessary condition for coping with stressors. In J. C. Quick \& L. E. Tetrick (Eds.), Handbook of Occupational Health Psychology (pp. 201-220). Washington, D.C.: APA.

Thobaben, M. (2007). Horizontal workplace violence. Home health Care Management \& Practice, 20(1), 82-83.

Thomas, K. W. (1992). Conflict and conflict management: Reflections and update. Journal of Organizational Behavior, 13, 265-274.

Thomas, L. T., \& Ganster, D. C. (1995). Impact of family-supportive work variables on work-family conflict and strain: A control perspective. Journal of Applied Psychology, 80, 6-15

Tjosvold, D. (1991). The conflict positive organization: Stimulate diversity and create unity. Reading, MA: Addison-Wesley.

Tjosvold, D. (1998). Cooperative and competitive goal approach to conflict: Accomplishments and challenges. Applied Psychology: An International Review, 47, 285-342.

Tse, H. H. M., Dasborough, M. T., \& Ashkanasy, N. M. (2008). A multi-level analysis of team climate and interpersonal exchange relationships at work. The Leadership Quarterly, 19, 195-211.

Todd, M., Tennen, H., Carney, M. A., Armeli, S., \& Affleck, G. (2004). Do we know how we cope? Relating daily coping reports to global and time-limited retrospective assessments. Journal of Personality and Social Psychology, 86(2), $310-319$.

Trinkoff, A. M., \& Storr, C. L. (1998). Substance use among nurses: Differences between specialties. American Journal of Public Health, 88(4), 581-585.

Trinkoff, A. M., Zhou, Q., \& Storr, C. L. (1999). Estimation of the prevalence of substance use problems among nurses using capture-recapture methods. Journal of Drug Issues, 29(1), 187-198.

Trinkoff, A.M., Zhou, Q., Storr, C. L., \& Soeken, K. L. (2000). Workplace access, negative proscriptions, job strain, and substance use in registered nurses. Nursing Research, 49(2), 83-90.

Tyler, P., \& Cushway, D. (1992). Stress, coping and mental well-being in hospital nurses. Stress Medicine, 8, 91-98.

Vaillant, G.E. (1977). Adaptation to life. Boston: Little \& Brown.

Valsiner, J., \& Cairns, R. B. (1992). Theoretical perspectives on conflict and development. In C. U. Shantz\&W.W. Hartup (Eds.), Conflict in child and adolescent development (pp. 15-35). New York: Cambridge University Press.

van den Broeck, A., de Cuyper, N., de Witte, H., \& Vansteenkiste, M. (2010). Not all job demands are equal: Differentiating job hindrances and job challenges in the Job Demands-Resources Model. European Journal of Work and Organizational Psychology, 19(6), 735-359. 
van der Doef, M., \& Maes, S. (1999). The Job Demand-Control (Support) Model and psychological well-being: A review of 20 years of empirical research. Work \& Stress, 13(2), 87-114.

van Vegchel, N., de Jonge, J., Bosma, H., \& Schaufeli, W. (2005). Reviewing the EffortReward Imbalance Model: Drawing up the balance of 45 empirical studies. Social Science \& Medicine, 50(5), 1117-1131.

Vitaliano, P. P., DeWolfe, D. J., Maiuro, R. D., Russo, J., \& Katon, W. (1990). Appraised changeability of a stressor as a modifier of the relationship between coping and depression: A test of the hypothesis of fit. Journal of Personality and Social Psychology, 59, 582-592.

Wall, J. A. Jr., \& Callister, R. R. (1995). Conflict and its management. Journal of Management, 21, 515-558.

Walster, E., Walster, G., \& Berscheid, E. (1978). Equity: Theory and research. Boston: Allyn and Bacon.

Wanous, J.P., \& Hudy, M.J. (2001). Single-item reliability: A replication and extension. Organizational Research Methods, 4, 361-375.

Wanous, J.P., Reichers, A.E., Hudy, M.J. (1997). Overall job satisfaction: How good are single-item measures? Journal of Applied Psychology, 82(2), 247-252.

Ware, J. E. Jr. (1978). Effects of acquiescent response set on patient satisfaction ratings. Medical Care, 16(4), 327-336.

Welbourne, J. L., Eggerth, D., Hartley, T. A., Andrew, M. E., \& Sanchez, F. (2007). Coping strategies in the workplace: Relationships with attributional style and job satisfaction. Journal of Vocational Behavior, 70, 312-325.

West, S. \& Hepworth, J.T. (1991). Statistical issues in the study of temporal data: Daily experiences. Journal of Personality, 59(3), 609-662.

Williams, C. (2003). Sources of workplace stress. Perspectives on Labour and Income, 4, 5-16.

Wright, R. R. (2009). Evaluation of the workplace interpersonal conflict construct among nurses using a mixed-method design: Comparison of an existing typology and an emergent thematic framework. Unpublished masters thesis. Portland State University, Portland.

Wright, T. A., \& Bonett, D. G. (1993). Role of employee coping and performance in voluntary employee withdrawal: A research refinement and elaboration. Journal of Management, 19(1), 147-161.

Xianyu, Y. \& Lambert, V.A. (2006). Investigation of the relationships among workplace stressors, ways of coping, and the mental health of Chinese head nurses. Nursing and Health Sciences, 8, 147-155.

Zajonc, R. B. (1980). Feeling and thinking: Preferences need no inferences. American Psychologist, 35(2), 151-175.

Zakowski, S. G., Hall, M. H., Klein, L. C., \& Baum, A. (2001). Appraised control, coping, and stress in a community sample: A test of the goodness of fit hypothesis. Annals of BehavioralMedicine, 23, 158-165. 
Zapf, D., \& Gross, C. (2001). Conflict escalation and coping with workplace bullying: A replication and extension. European Journal of Work and Organizational Psychology, 10(4), 497-522.

Zeidner, M., \& Saklofske, D. (1996). Adaptive and maladaptive coping. In M. Zeidner, \& S. Norman (Eds.), Handbook of coping: Theory, research, applications. (pp. 505531). Oxford, England: John Wiley \& Sons.

Zillman, D. (1994). Cognition-excitation interdependencies in the escalation of anger and angry aggression. In Potegal M, Knutson JF (Eds.), The Dynamics of Aggression: Biological and Social Process (pp. 45-75). Hillsdale, NJ: Erlbaum.

Zimber, A, Rudolf, A, \& Teufel, S. (2001). A training program to reduce distress among geriatric caregivers. Zeitschrift fur Gerontologie und Geriatrie, 34(5), 401-407.

Zohar, D. (2003). The influence of leadership and climate on occupational health and safety. In D. A. Hoffman \& L. E. Tetrick (Eds.), Health and safety in organizations: A multilevel perspective (pp. 201-230). San Francisco: JosseyBass.-R. 


\section{Appendix A: Glossary of Acronyms}

\begin{tabular}{|l|l|}
\hline Acronym & Meaning \\
\hline AIC & Akaike's Information Criteria \\
\hline ANOVA & Analysis of Variance \\
\hline CFA & Confirmatory Factor Analysis \\
\hline CFI & Compariative Fit Index \\
\hline COPE & Not an acronym - name of a coping measure \\
\hline Co-PI & Co-Principal Investigator \\
\hline COR & Conservation of Resources Theory \\
\hline CWB & Counterproductive Work Behavior \\
\hline$d f$ & Degrees of Freedom \\
\hline EF & Emotion-Focused \\
\hline ERI & Effort-Reward Imbalance Model \\
\hline HC & Humor Coping \\
\hline HLM & Hierarchical Linear Modeling, a statistical program \\
\hline Hoelter's CN & Hoelter's Critical Number \\
\hline ICC & Intraclass Coefficient \\
\hline IFI & Incremental Fit Index \\
\hline IRB & Institutional Review Board \\
\hline JDC & Job Demands-Control Model \\
\hline JDR & Job Demands-Resources Model \\
\hline MAR & Missing at Random \\
\hline MCAR & Missing Completely at Random \\
\hline NFI & Normed Fit Index \\
\hline NIM & Non-ignorable Missing \\
\hline NIOSH & National Institute of Occupational Safety and Health \\
\hline ONA & Oregon Nurses Association \\
\hline ONRP & Oregon Nurse Retention Project \\
\hline PF & Problem-Focused \\
\hline PI & Principal Investigator \\
\hline RC & Religious Coping \\
\hline RMSEA & Root Mean Square Error of Approximation \\
\hline RN & Registered Nurse \\
\hline SD & Standard Deviation \\
\hline WCC & Ways of Coping Checklist \\
\hline WCQ & Ways of Coping Questionnaire \\
\hline WIC & \\
\hline
\end{tabular}




\title{
Appendix B: The Oregon Nurse Retention Project Demographics and Weekly Survey
}

\section{Oregon Nurse Retention Project Demographics Survey}

Welcome! This web page is the site to register for the Oregon Nurse Retention Project. The purpose of this research is to study the factors that cause nurses to leave their jobs as well as the work experiences that inspire them to stay on their jobs. This project is being conducted by the research team of Dr. Robert Sinclair, Portland State University in collaboration with the Professional Services Program, Oregon Nurses Association. The research is paid for by a grant from the Northwest Health Foundation.

The information gathered on this page will be used to identify and contact our participants for the actual research and to ensure that participants receive any financial or other compensation earned by participating. All information you provide will be stored in a secure location. The researcher may match your survey responses to your demographic information to be able to describe the people who participate and to understand patterns of responses. This information may also be used to match responses from people who complete one survey to other surveys they complete. However, no records from this research or reports of this research in any form will link your name or other personally identifying information to survey responses.

If you have further questions about the study, please feel free to contact Dr. Sue Davidson at 503-293-0011 x 320, or via e-mail at davidson@ oregonrn.org. You also may contact Dr. Robert Sinclair with questions at 503-725-3965 or via e-mail at $\underline{\text { sinclair@pdx.edu. }}$

If you are interested in participating in this project, please click next on the page below. You will be asked to complete a brief set of questions about your personal and work background. Please complete each of the questions that follow by checking or indicating your response.

\section{Individual information}

1. What is your gender?

$\checkmark$ Male

$\square$ Female

Other

2. What is your age in years?

3. What is your ethnicity? (Check all that apply)

\author{
American Indian or Alaskan Native \\ $\square$ Asian \\ $\square$ Black or African American \\ $\square$ Hispanic or Latino/Latina \\ $\square$ Native Hawaiian or other Pacific Islander \\ $\square$ White \\ $\square$ Multi-ethnic
}


4. What city do you most frequently work in?

\section{Family Information}

1. How many children do you have (including stepchildren) living at home?

2. Are you providing care to any adult (age 18 or over)?

$\square$ Yes; go to Question 3
$\square$ No; go to Question 4

3. Indicate whether you are providing care for others in these specific examples.

Yes

No

Are you providing care for a parent

or parent-in-law?

Are you providing care for a disabled spouse?

Are you providing care for some other disabled adult?

4. What is your current relationship status?

$\square$ Married

$\square$ Divorced or separated

$\square$ Widowed

$\square$ Living with a significant other

$\square$ Never married

Other

\section{Work Information}

1. What is your HIGHEST level of education?

$\square$ Diploma in Nursing

$\square$ Associate Degree in Nursing

$\square$ Associate Degree, Non-nursing

$\square$ Baccalaureate Degree, Nursing

$\square$ Baccalaureate Degree, Non-nursing

$\square$ Masters Degree, Nursing

$\square$ Masters Degree, Non-nursing

$\square$ Doctorate in Nursing 
$\square$ Doctorate, Non-nursing

2. Which of the following health care settings is your PRIMARY/USUAL place of work? (Choose one answer).

$\square$ Ambulatory/outpatient clinic/MD office

$\square$ Business, industry

Community or public health agency

Home Health agency

Hospital/acute care facility

Long-term care facility

$\square$ Public/private school (K-12)

$\checkmark$ School of nursing

Government agency

Other

3. Do you have a secondary health care setting where you work? (Choose one answer).

$\square$ Ambulatory/outpatient clinic/MD office

$\square$ Business, industry

$\square$ Community or public health agency

Home Health agency

Hospital/acute care facility

Long-term care facility

$\square$ Public/private school (K-12)

$\square$ School of nursing

$\square$ Government agency

$\square$ Other

4. What is your PRIMARY nursing specialty area? (Choose one)

$\square$ Behavioral Health

Community/Public Health

Critical Care/NICU

Emergency/Trauma

$\square$ End of Life/Palliative Care

$\square$ Family Nursing

General Medical, General Surgical

Gerontology

Home Health

Maternal Child/Obstetrics

Occupational Health

$\square$ Operating Room, PACU 
$\square$ Pain Management

$\square$ Pediatrics

Psychiatry

$\square$ School Health

$\square$ Women's Health

Other (please specify)

5. What is your PRIMARY position or role in nursing?

$\square$ Academic faculty

$\square$ Case manager

$\square$ Clinical nurse specialist

Consultant

$\square$ Manager or supervisor

$\square$ Nurse executive or nurse administrator

$\square$ Nurse midwife

$\square$ Nurse practitioner

$\square$ Staff nurse

$\square$ Staff development/clinical educator

$\square$ Other

6. What is your work status in your primary job? (Choose one answer).

$\square$ Full-time

$\square$ Part-time

$\square$ Resource

7. Does your primary position involve any call?

$\square$ Yes, voluntary

$\square$ Yes, mandatory call is part of the position

$\square$ No, no call at all

8. What shift do you usually work in your primary position?

$\square$ Days

$\square$ Evenings

$\square$ Nights

Other (please specify)

9. How many hours are there in your agreed-upon shift?

$\square 4$ hour shift

$\square 8$ hour shift

$\square 9$ hour shift

$\square 10$ hour shift 
$\square 12$ hour shift

Other (please specify)

10. In a typical employer defined work week, how many hours are you SCHEDULED to work?

11. In a typical employer defined work week, how many hours do you ACTUALLY WORK per week?

12. Typically, how much VOLUNTARY OVERTIME (in approximate hours) do you work per week?

13. Typically, approximately how many shifts do you work per week?

14. Are you in a benefited position?

$\square$ Yes

$\square$ No

$\square$ Unsure

15. How long have you been a registered nurse? (years)

16. How many years has it been since you completed your basic nursing program that lead to your licensure as an RN? (years)

17. How long have you been working in your current organization? (years)

18. How long have you been working in your current position? (years)

19. Your first name

20. Your last name 
21. Your phone number

22. My e-mail address

23. My address is (street, city, zip, state)

24. In completing other surveys in the various sections of this project, which format would you prefer?

$\square$ Hard copy

$\square$ Web-based 


\section{The Oregon Nurse Retention Project Weekly Survey}

You are invited to participate in a research study conducted by Dr. Robert Sinclair and Dr. Cynthia Mohr from the Department of Psychology at Portland State University (PSU) in collaboration with the Oregon Nurses Association (ONA). This study will investigate the kinds of positive and stressful experiences you have at work and your attitudes and perceptions about yourself at work, your organization, and the profession of nursing. The general goal of the study is to investigate how occupational stress and positive experiences at work influence nurses' desires to stay in the profession of nursing as well as their thoughts about leaving the profession. We will compile a series of recommendations based on our findings across the entire group of participants and will report these findings to acute care facilities. In doing so, we hope to help create healthier and more rewarding work environments for nurses.

\section{What will I have to do?}

- If you decide to participate, you will be asked to complete web-based versions of the surveys.

- You will be asked to answer 12 brief weekly surveys regarding your nursing work life, including your perspectives on the field of nursing, your career as a nurse, the organization you work for, the people you work with, your current job, and some questions about you as a person.

- These weekly surveys take approximately 15-25 minutes to complete.

- You may contact the researchers throughout the study via email (sinclair@pdx.edu) and/or telephone (503) 725-3986 to ask any questions you have and address any problems you might be having.

\section{Are there any risks?}

- There is no direct cost associated with your completion of the surveys in this study. There are no anticipated physical or psychological risks from participating in this study. It is possible that you may be upset by the recollection of unpleasant experiences. However, our past experience with questionnaire research suggests this is unlikely. Moreover, you will be able to avoid answering any question that makes you uncomfortable.

\section{Your participation is voluntary.}

- Your participation in this study is completely voluntary. You are under no obligation to participate and choosing not to participate will not affect your relationship with Portland State University or the Oregon Nurses Association.

- You may choose to not answer questions or withdraw from participating in this study at any time.

- If you demonstrate difficulty in completing the weekly surveys, you may be terminated from the study. In this case, you will be paid for surveys completed prior to the termination date.

\section{What will I get in return?}

- In exchange for your participation, you will receive $\$ 5$ for each completed weekly survey.

- In addition to the $\$ 5$ per survey, you will be entered in a raffle for $\$ 50$ for each four week period of the survey you complete, as well as another $\$ 50$ raffle for participants who complete all 12 weeks of the survey.

- Thus, you can earn $\$ 60-\$ 260$ for participating in this study.

- A full report on the purpose of and findings of this study will be published and freely available. We will 
provide a copy of this report to all study participants at no charge.

\section{What are you doing to protect me?}

Any information that is obtained in connection with this study and that can be linked to you or identify you will be kept confidential. Confidentiality of your responses will be maintained through several means:

- Information about your identity will be kept in a separate (secure and password-protected) database from your responses to the survey questions. Data from the surveys will be identified by code numbers that only will be able to be accessed by research personnel.

- Your responses cannot be accessed from the web without a password; only research personnel will be able to download your responses.

- As soon as responses are downloaded there will be no online access to responses. Also, any information that you provide in your responses that could potentially identify you, such as if you named your facility, will be deleted from any reports that include those responses.

\section{Any questions?}

Please contact the Human Subjects Research Review Committee, Office of Research and Sponsored Projects, 111 Cramer Hall, Portland State University, (503) 725-4288. If you have questions about the study itself, please contact Dr. Robert Sinclair by telephone at (503) 725-3986, by e-mail at sinclair@pdx.edu, or by mail at Department of Psychology, P.O. Box 751, Portland State University, Portland, OR 97207. Or, you may contact Dr. Sue Davidson at the Oregon Nurses Association by telephone at (503) 293-0011, by e-mail at davidson@oregonm.org , or by mail at Oregon Nurses Association, 18765 SW Boones Ferry Rd., Ste 200, Tualatin, OR 97062.

\section{You may wish to print a copy of this letter to keep for your records.}

In order to participate, you must select "Yes" below. If you do not wish to participate, simply close this window.

C Yes, I wish to participate in the study. 
In the following table, please fill out a row for each shift that you worked in the past 7 days.

\begin{tabular}{|c|c|c|c|c|}
\hline Date & $\begin{array}{l}\text { Start } \\
\text { Time }\end{array}$ & $\begin{array}{l}\text { Stop } \\
\text { Time }\end{array}$ & $\begin{array}{c}\text { Were you sent home } \\
\text { because of patient } \\
\text { census? }\end{array}$ & Shift Type \\
\hline- & $\mathrm{am} / \mathrm{pm}$ & $\mathrm{am} / \mathrm{pm}$ & $\begin{array}{l}\square \quad \text { Yes } \\
\square \quad \text { No }\end{array}$ & $\begin{array}{l}\text { Scheduled } \\
\square \text { Called In } \\
\square \text { Scheduled OT }\end{array}$ \\
\hline- & $\mathrm{am} / \mathrm{pm}$ & $\mathrm{am} / \mathrm{pm}$ & $\begin{array}{ll}\square \quad \text { Yes } \\
\square \quad \text { No }\end{array}$ & $\begin{array}{l}\square \text { Scheduled } \\
\square \text { Called In } \\
\square \text { Scheduled OT }\end{array}$ \\
\hline-1 & $\mathrm{am} / \mathrm{pm}$ & $\mathrm{am} / \mathrm{pm}$ & $\begin{array}{ll}\square \quad \text { Yes } \\
\square \quad \text { No }\end{array}$ & $\begin{array}{l}\text { Scheduled } \\
\square \text { Called In } \\
\square \text { Scheduled OT }\end{array}$ \\
\hline-1 & $\mathrm{am} / \mathrm{pm}$ & $\mathrm{am} / \mathrm{pm}$ & $\begin{array}{ll}\square \quad \text { Yes } \\
\square \quad \text { No }\end{array}$ & $\begin{array}{l}\text { Scheduled } \\
\square \text { Called In } \\
\square \text { Scheduled OT }\end{array}$ \\
\hline $\begin{array}{l}1 \\
-\end{array}$ & $\mathrm{am} / \mathrm{pm}$ & $\mathrm{am} / \mathrm{pm}$ & $\begin{array}{ll}\square \quad \text { Yes } \\
\square \quad \text { No }\end{array}$ & $\begin{array}{l}\square \text { Scheduled } \\
\square \text { Called In } \\
\square \text { Scheduled OT }\end{array}$ \\
\hline-1 & $\mathrm{am} / \mathrm{pm}$ & $\mathrm{am} / \mathrm{pm}$ & $\begin{array}{ll}\square \quad \text { Yes } \\
\square \quad \text { No }\end{array}$ & $\begin{array}{l}\square \text { Scheduled } \\
\square \text { Called In } \\
\square \text { Scheduled OT }\end{array}$ \\
\hline-1 & $\mathrm{am} / \mathrm{pm}$ & $\mathrm{am} / \mathrm{pm}$ & $\begin{array}{l}\square \quad \text { Yes } \\
\square \quad \text { No }\end{array}$ & $\begin{array}{l}\square \text { Scheduled } \\
\square \text { Called In } \\
\square \text { Scheduled OT }\end{array}$ \\
\hline
\end{tabular}

How many on call hours did you work in the past 7 days? (hours)

\begin{tabular}{|l|c|c|c|c|c|c|c|}
\hline \multicolumn{7}{|l|}{ Over the past 7 days, during how many shifts did the following events occur? } \\
\hline & $\begin{array}{c}0 \\
\text { Shifts }\end{array}$ & $\begin{array}{c}1 \\
\text { Shift }\end{array}$ & $\begin{array}{c}2 \\
\text { Shifts }\end{array}$ & $\begin{array}{c}3 \\
\text { Shifts }\end{array}$ & $\begin{array}{c}4 \\
\text { Shifts }\end{array}$ & $\begin{array}{c}5 \\
\text { Shifts }\end{array}$ & $\begin{array}{c}6+ \\
\text { Shifts }\end{array}$ \\
\hline $\begin{array}{l}\text { I provided emotional support to my } \\
\text { patient/patient's family. }\end{array}$ & & & & & & & \\
\hline $\begin{array}{l}\text { I developed a close bond with my } \\
\text { patient. }\end{array}$ & & & & & & & \\
\hline I helped my patient die with dignity. & & & & & & & \\
\hline $\begin{array}{l}\text { I had a patient whose condition } \\
\text { unexpectedly improved. }\end{array}$ & & & & & & & \\
\hline I helped save the life of a patient. & & & & & & & \\
\hline $\begin{array}{l}\text { I realized I made a difference in } \\
\text { someone else's life. }\end{array}$ & & & & & & & \\
\hline $\begin{array}{l}\text { I helped my patient physically feel } \\
\text { better. }\end{array}$ & & & & & & & \\
\hline
\end{tabular}




\begin{tabular}{|l|l|l|l|l|l|l|l|}
\hline $\begin{array}{l}\text { I successfully implemented a } \\
\text { challenging procedure for my patient. }\end{array}$ & & & & & & & \\
\hline $\begin{array}{l}\text { I taught my patient a complex self-care } \\
\text { task. }\end{array}$ & & & & & & & \\
\hline $\begin{array}{l}\text { I educated my patient/family about } \\
\text { his/her condition(s). }\end{array}$ & & & & & & & \\
\hline $\begin{array}{l}\text { I figured out how to perform a difficult } \\
\text { task. }\end{array}$ & & & & & & & \\
\hline I overcame a challenge at work. & & & & & & & \\
\hline
\end{tabular}

\begin{tabular}{|l|c|c|c|c|c|c|c|}
\hline Over the past 7 days, during how many shifts did the following events occur? \\
\hline & $\begin{array}{c}0 \\
\text { Shifts }\end{array}$ & $\begin{array}{c}1 \\
\text { Shift }\end{array}$ & $\begin{array}{c}2 \\
\text { Shifts }\end{array}$ & $\begin{array}{c}3 \\
\text { Shifts }\end{array}$ & $\begin{array}{c}4 \\
\text { Shifts }\end{array}$ & $\begin{array}{c}5 \\
\text { Shifts }\end{array}$ & $\begin{array}{c}6+ \\
\text { Shifts }\end{array}$ \\
\hline A physician complimented my work. & & & & & & & \\
\hline A coworker complimented my work. & & & & & & & \\
\hline My manager complimented my work. & & & & & & & \\
\hline $\begin{array}{l}\text { A coworker and/or manager gave me } \\
\text { helpful feedback about my work. }\end{array}$ & & & & & & & \\
\hline $\begin{array}{l}\text { My coworkers and I worked well as a } \\
\text { team. }\end{array}$ & & & & & & & \\
\hline $\begin{array}{l}\text { At work, my coworkers and I shared a } \\
\text { laugh about something. }\end{array}$ & & & & & & & \\
\hline $\begin{array}{l}\text { People in my unit went out of their way } \\
\text { to be nice to each other. }\end{array}$ & & & & & & & \\
\hline $\begin{array}{l}\text { Other nurses shared knowledge with me } \\
\text { about nursing practice. }\end{array}$ & & & & & & & \\
\hline $\begin{array}{l}\text { A coworker taught me effective ways to } \\
\text { deal with people. }\end{array}$ & & & & & & & \\
\hline $\begin{array}{l}\text { My coworker taught me an effective } \\
\text { technique or strategy. }\end{array}$ & & & & & & & \\
\hline $\begin{array}{l}\text { Another nurse helped me when I really } \\
\text { needed it. }\end{array}$ & & & & & & & \\
\hline $\begin{array}{l}\text { My manager helped me when I really } \\
\text { needed it. }\end{array}$ & & & & & & & \\
\hline $\begin{array}{l}\text { A physician helped me when I really } \\
\text { needed it. }\end{array}$ & & & & & & & \\
\hline
\end{tabular}

\begin{tabular}{|l|c|c|c|c|c|c|c|}
\hline Over the past 7 days, during how many shifts did the following events occur? \\
\hline & $\begin{array}{c}0 \\
\text { Shifts }\end{array}$ & $\begin{array}{c}1 \\
\text { Shift }\end{array}$ & $\begin{array}{c}2 \\
\text { Shifts }\end{array}$ & $\begin{array}{c}3 \\
\text { Shifts }\end{array}$ & $\begin{array}{c}4 \\
\text { Shifts }\end{array}$ & $\begin{array}{c}5 \\
\text { Shifts }\end{array}$ & $\begin{array}{c}6+ \\
\text { Shifts }\end{array}$ \\
\hline A patient thanked me for my work. & & & & & & & \\
\hline $\begin{array}{l}\text { A patient's family member(s) thanked } \\
\text { me for my work. }\end{array}$ & & & & & & & \\
\hline A physician thanked me for my work. & & & & & & & \\
\hline $\begin{array}{l}\text { My charge nurse thanked me for my } \\
\text { work. }\end{array}$ & & & & & & & \\
\hline A coworker thanked me for my work. & & & & & & & \\
\hline
\end{tabular}




\begin{tabular}{|l|l|l|l|l|l|l|l|}
\hline $\begin{array}{l}\text { I shared knowledge about nursing } \\
\text { practice with a coworker. }\end{array}$ & & & & & & & \\
\hline $\begin{array}{l}\text { I helped a fellow nurse when s/he } \\
\text { needed me. }\end{array}$ & & & & & & & \\
\hline $\begin{array}{l}\text { I responded to the emotional needs of a } \\
\text { fellow worker. }\end{array}$ & & & & & & & \\
\hline
\end{tabular}

During the past 7 days, what has been the most positive/beneficial/rewarding event that occurred at your primary job? Please describe it below:

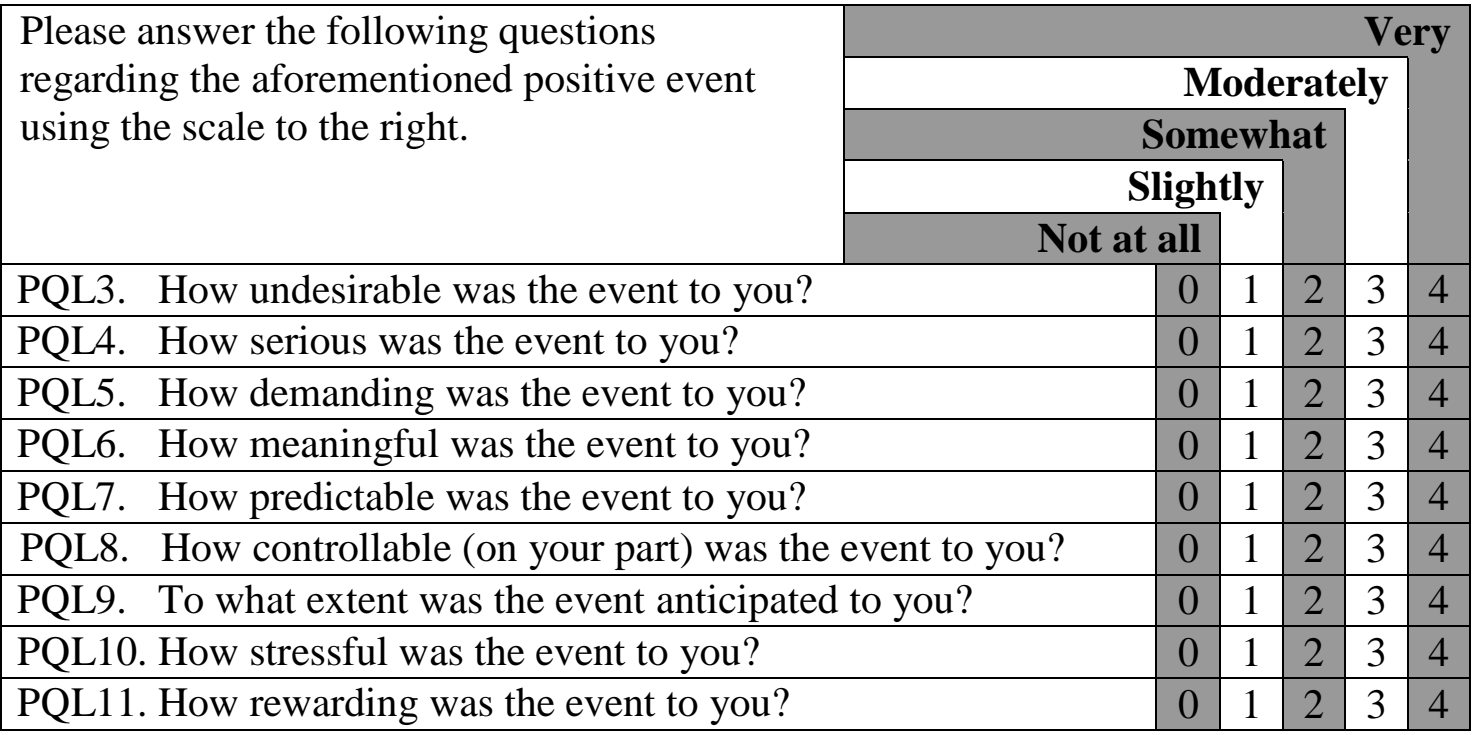

What, if anything, could your organization do to increase the likelihood that this event will occur again in the future? 


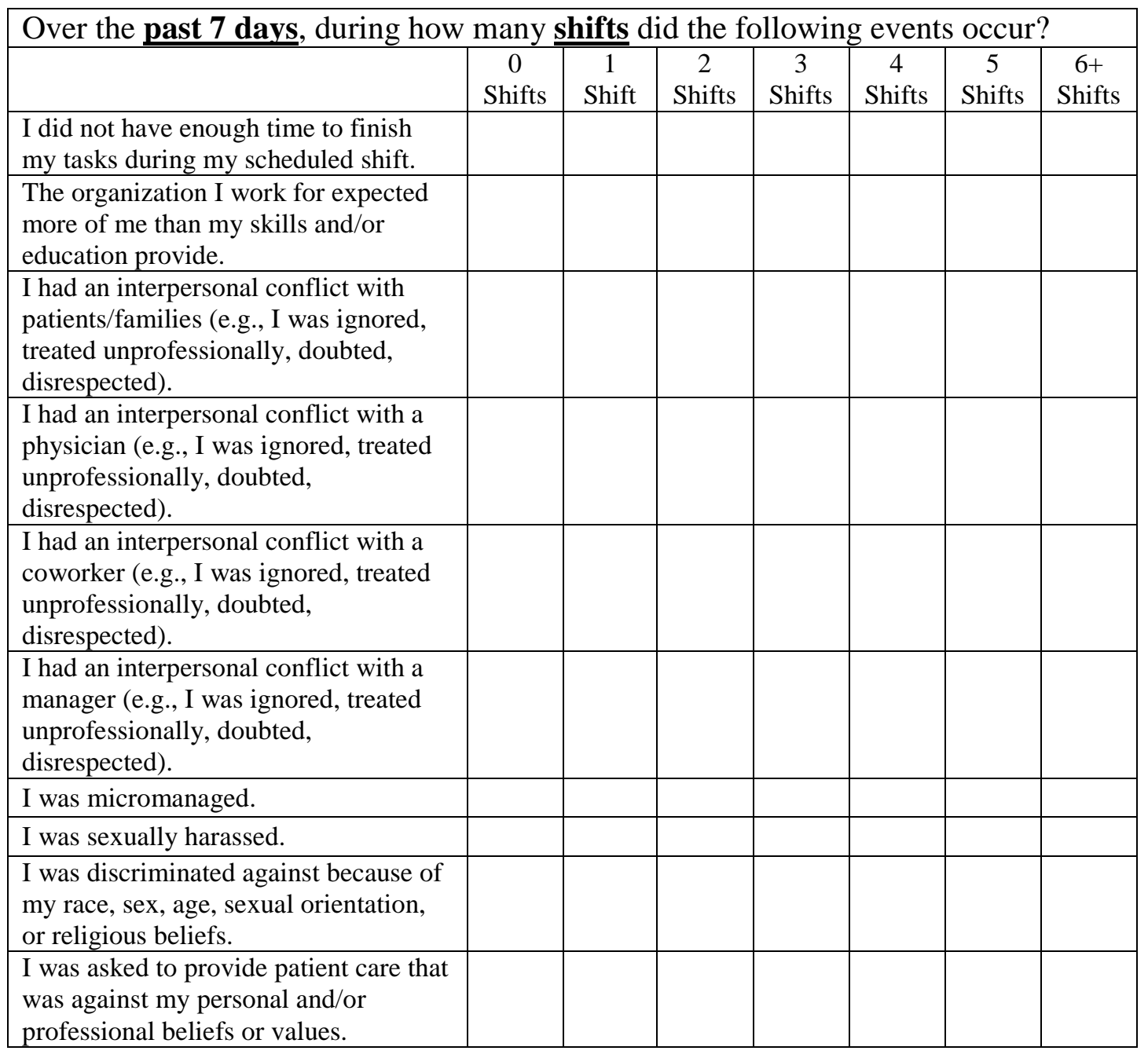

\begin{tabular}{|l|c|c|c|c|c|c|c|}
\hline Over the past 7 days, during how many & \multicolumn{7}{|c|}{ shifts did the following events occur? } \\
\hline & $\begin{array}{c}0 \\
\text { Shifts }\end{array}$ & $\begin{array}{c}1 \\
\text { Shift }\end{array}$ & $\begin{array}{c}2 \\
\text { Shifts }\end{array}$ & $\begin{array}{c}3 \\
\text { Shifts }\end{array}$ & $\begin{array}{c}4 \\
\text { Shifts }\end{array}$ & $\begin{array}{c}5 \\
\text { Shifts }\end{array}$ & $\begin{array}{c}6+ \\
\text { Shifts }\end{array}$ \\
\hline $\begin{array}{l}\text { I felt helpless when a patient failed to } \\
\text { improve. }\end{array}$ & & & & & & & \\
\hline $\begin{array}{l}\text { I had a patient whose health } \\
\text { unexpectedly declined. }\end{array}$ & & & & & & & \\
\hline $\begin{array}{l}\text { I received incomplete or unclear } \\
\text { information about a patient's } \\
\text { condition. }\end{array}$ & & & & & & & \\
\hline $\begin{array}{l}\text { I experienced problems with } \\
\text { equipment or supplies (e.g., } \\
\text { computer system problems, }\end{array}$ & & & & & & & \\
\hline
\end{tabular}




\begin{tabular}{|l|l|l|l|l|l|l|l|}
\hline $\begin{array}{l}\text { unfamiliar equipment, misplaced } \\
\text { supplies). }\end{array}$ & & & & & & & \\
\hline $\begin{array}{l}\text { I did not have enough RNs with the } \\
\text { specific education or skills needed } \\
\text { for this unit. }\end{array}$ & & & & & & & \\
\hline $\begin{array}{l}\text { I did not have enough experienced } \\
\text { RNs to take care of patient needs. }\end{array}$ & & & & & & & \\
\hline $\begin{array}{l}\text { I did not have enough RNs to meet } \\
\text { patient care demands. }\end{array}$ & & & & & & & \\
\hline $\begin{array}{l}\text { I did not have enough staff to } \\
\text { adequately cover the unit. }\end{array}$ & & & & & & & \\
\hline $\begin{array}{l}\text { Scheduled staff were late or absent } \\
\text { for a shift. }\end{array}$ & & & & & & & \\
\hline $\begin{array}{l}\text { Requests for additional staff were } \\
\text { denied. }\end{array}$ & & & & & & & \\
\hline $\begin{array}{l}\text { Requested staff were approved but } \\
\text { did not arrive in time. }\end{array}$ & & & & & & & \\
\hline
\end{tabular}

\begin{tabular}{|l|c|c|c|c|c|c|c|}
\hline \multicolumn{1}{|c|}{ Over the past 7 days, during how many shifts did the following events occur? } \\
\hline & $\begin{array}{c}0 \\
\text { Shifts }\end{array}$ & $\begin{array}{c}1 \\
\text { Shift }\end{array}$ & $\begin{array}{c}2 \\
\text { Shifts }\end{array}$ & $\begin{array}{c}3 \\
\text { Shifts }\end{array}$ & $\begin{array}{c}4 \\
\text { Shifts }\end{array}$ & $\begin{array}{c}5 \\
\text { Shifts }\end{array}$ & $\begin{array}{c}6+ \\
\text { Shifts }\end{array}$ \\
\hline I missed my rest break. & & & & & & & \\
\hline I missed my meal break. & & & & & & & \\
\hline $\begin{array}{l}\text { I worked voluntary overtime (extended } \\
\text { shift). }\end{array}$ & & & & & & & \\
\hline $\begin{array}{l}\text { I worked mandatory overtime } \\
\text { (extended shift). }\end{array}$ & & & & & & & \\
\hline $\begin{array}{l}\text { I worked pressured (but not mandated) } \\
\text { overtime (extended shift). }\end{array}$ & & & & & & & \\
\hline $\begin{array}{l}\text { I was sent home because of low patient } \\
\text { census. }\end{array}$ & & & & & & & \\
\hline $\begin{array}{l}\text { I had to stay after my scheduled shift } \\
\text { because no one came in to take over my } \\
\text { care. }\end{array}$ & & & & & & & \\
\hline $\begin{array}{l}\text { I had to stay after my shift to finish my } \\
\text { work (charting, etc.). }\end{array}$ & & & & & & & \\
\hline I worked an overtime shift. & & & & & & & \\
\hline
\end{tabular}

In the past 7 days, what has been the most negative/stressful event that occurred at your primary job? It can include things such as safety hazards, equipment or supply issues, staffing, dealing with death/dying, workload, or ethics/professionalism. Please describe it below: 


\begin{tabular}{|c|c|c|c|c|c|}
\hline \multirow{5}{*}{$\begin{array}{l}\text { Please answer the following questions about } \\
\text { the aforementioned event using the scale to the } \\
\text { right. }\end{array}$} & & & & \multicolumn{2}{|c|}{ Very } \\
\hline & \multicolumn{5}{|c|}{ Moderately } \\
\hline & \multicolumn{3}{|c|}{ Somewhat } & & \\
\hline & \multicolumn{3}{|c|}{ Slightly } & & \\
\hline & it all & & & & \\
\hline NQL3. How undesirable was the event to you? & 0 & 1 & 2 & 3 & 4 \\
\hline NQL4. How serious was the event to you? & 0 & 1 & 2 & 3 & 4 \\
\hline NQL5. How demanding was the event to you? & 0 & 1 & 2 & 3 & 4 \\
\hline NQL6. How meaningful was the event to you? & 0 & 1 & 2 & 3 & 4 \\
\hline NQL7. How predictable was the event to you? & 0 & 1 & 2 & 3 & 4 \\
\hline NQL8. How controllable (on your part) was the event to you? & 0 & 1 & 2 & 3 & 4 \\
\hline NQL9. To what extent was the event anticipated to you? & 0 & 1 & 2 & 3 & 4 \\
\hline NQL10. How stressful was the event to you? & 0 & 1 & 2 & 3 & 4 \\
\hline NQL11. How rewarding was the event to you? & 0 & 1 & 2 & 3 & 4 \\
\hline
\end{tabular}

What, if anything, could your organization do to decrease the likelihood that this event will occur again in the future?

Take a moment to think about the most negative/stressful interpersonal

interaction/conflict you had in the past 7 days with someone at your primary job. An interpersonal conflict may include anything from minor disagreements to physical assaults. The conflict may be overt (e.g., coworker was directly being rude to you) or may be covert (e.g., coworker spread rumors about you). Please describe it below:

Is the interpersonal conflict the same event you rated under the most negative/stressful event?

$\square$ Yes

$\square$ No

Who was this interaction/conflict with? (Please select only one choice)

$\square$ Physician

$\square$ Nurse Manager

$\square$ Nurse Peer

$\square$ Other (e.g., patient, patient family) 
When did this event occur?

$\square$ Sunday

$\square$ Monday

$\square$ Tuesday

Wednesday

$\checkmark$ Thursday

$\square$ Friday

$\square$ Saturday

Please provide a brief description of this event including causes of the conflict.

Please answer the following questions regarding the above-mentioned interpersonal conflict using the scale below.

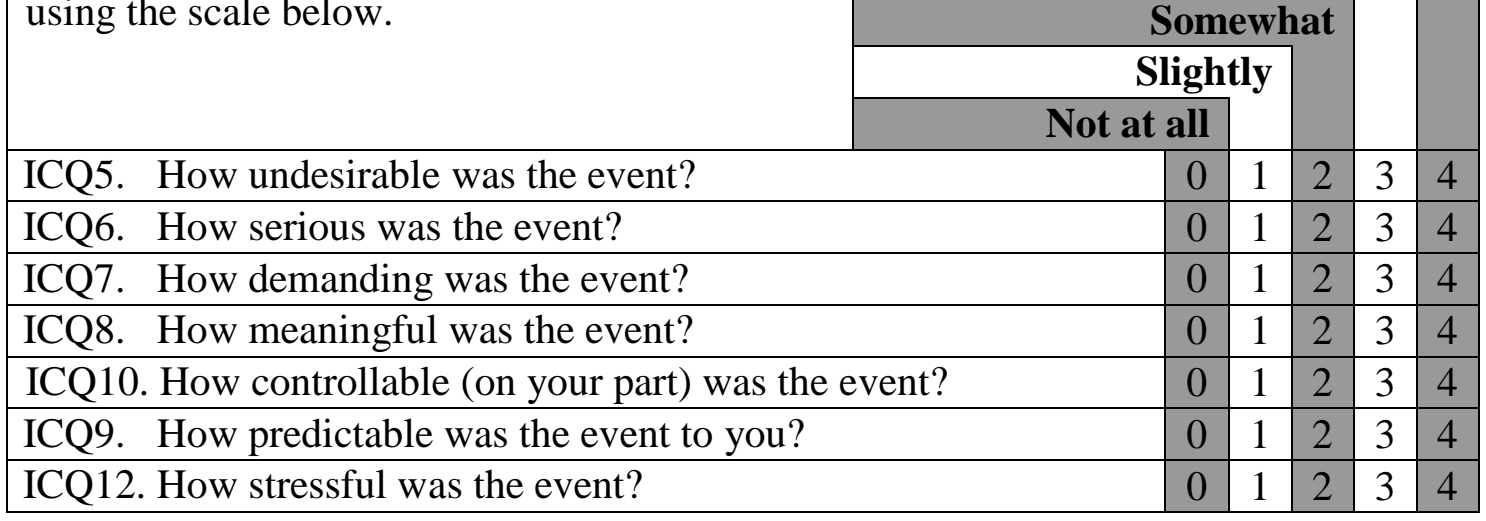

ICQ4. If anything, what could your organization do to decrease the likelihood of this occurring in the future? 
The following statements are ways people may respond when experiencing stressful events. Think about what you did with your above-identified most negative or stressful conflict this week at work and then indicate your response to each question below.

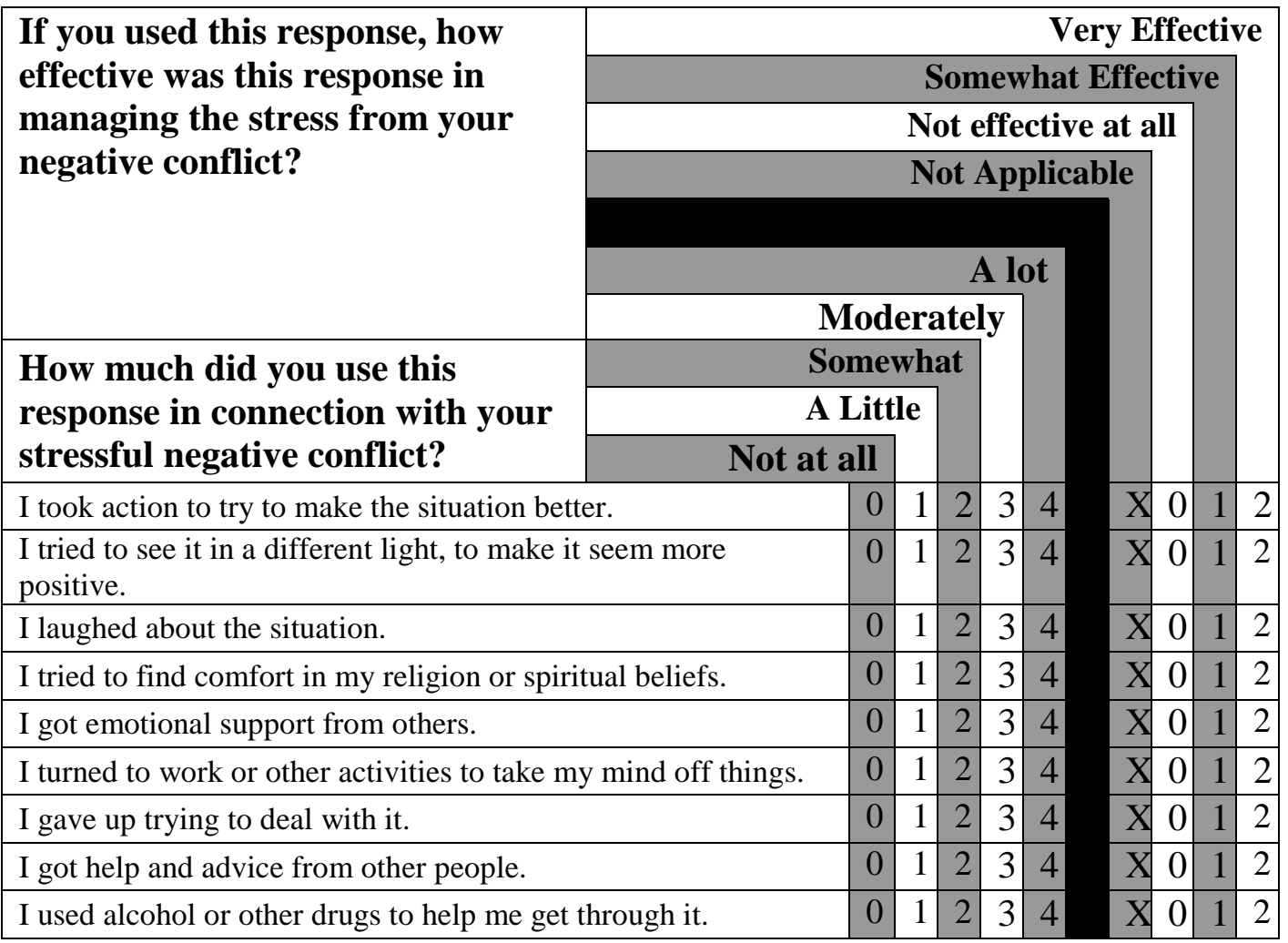

\begin{tabular}{|l|c|c|c|c|c|c|c|}
\hline Over the past 7 days, during how many shifts did the following events occur? \\
\hline & $\begin{array}{c}0 \\
\text { Shifts }\end{array}$ & $\begin{array}{c}1 \\
\text { Shift }\end{array}$ & $\begin{array}{c}2 \\
\text { Shifts }\end{array}$ & $\begin{array}{c}3 \\
\text { Shifts }\end{array}$ & $\begin{array}{c}4 \\
\text { Shifts }\end{array}$ & $\begin{array}{c}5 \\
\text { Shifts }\end{array}$ & $\begin{array}{c}6+ \\
\text { Shifts }\end{array}$ \\
\hline I was enthusiastic about my job. & & & & & & & \\
\hline My job inspired me. & & & & & & & \\
\hline I was proud of the work that I did. & & & & & & & \\
\hline $\begin{array}{l}\text { At my work, I felt bursting with } \\
\text { energy. }\end{array}$ & & & & & & & \\
\hline At my job, I felt strong and vigorous. & & & & & & & \\
\hline $\begin{array}{l}\text { When I got up in the morning, I felt } \\
\text { like going to work. }\end{array}$ & & & & & & & \\
\hline $\begin{array}{l}\text { I felt happy when I was working } \\
\text { intensely. }\end{array}$ & & & & & & & \\
\hline I was immersed in my work. & & & & & & & \\
\hline I was absorbed in my work. & & & & & & & \\
\hline I felt tired at work. & & & & & & & \\
\hline
\end{tabular}




\begin{tabular}{|l|l|l|l|l|l|l|l|}
\hline I felt physically drained at work. & & & & & & & \\
\hline $\begin{array}{l}\text { I felt like my "batteries" were "dead" } \\
\text { at work. }\end{array}$ & & & & & & & \\
\hline I had difficulty concentrating at work. & & & & & & & \\
\hline I was not thinking clearly at work. & & & & & & & \\
\hline $\begin{array}{l}\text { I was not focused in my thinking at } \\
\text { work. }\end{array}$ & & & & & & & \\
\hline $\begin{array}{l}\text { I was unable to be sensitive to the } \\
\text { needs of coworkers and patients. }\end{array}$ & & & & & & & \\
\hline $\begin{array}{l}\text { I was not capable of investing } \\
\text { emotionally in coworkers and patients. }\end{array}$ & & & & & & & \\
\hline $\begin{array}{l}\text { I was not capable of being } \\
\text { sympathetic to coworkers and } \\
\text { patients. }\end{array}$ & & & & & & & \\
\hline
\end{tabular}

Please indicate how often you experienced the following over the past 7 days on the scale below.

\begin{tabular}{|c|c|c|c|c|c|}
\hline I had trouble sleeping. & 1 & 2 & 3 & 4 & 5 \\
\hline I had a headache. & 1 & 2 & 3 & 4 & 5 \\
\hline I felt sick to my stomach/have indigestion or heartburn. & 1 & 2 & 3 & 4 & 5 \\
\hline I had a cold or flu. & 1 & 2 & 3 & 4 & 5 \\
\hline I had back or muscle aches. & 1 & 2 & 3 & 4 & 5 \\
\hline I had chest pain. & 1 & 2 & 3 & 4 & 5 \\
\hline I tried to eat healthy foods. & 1 & 2 & 3 & 4 & 5 \\
\hline I ate healthy foods. & 1 & 2 & 3 & 4 & 5 \\
\hline
\end{tabular}

In the past 7 days:

How often have you had trouble staying awake while driving, eating meals, or engaging in social activity?

$\square$ Not during the past week

$\square$ Once or twice this week

$\square$ Three or more times this week

How would you rate your sleep quality for the past 7 days overall?

$\square$ Very good

$\square$ Fairly good

$\square$ Fairly bad

$\square$ Very bad

In the past 7 nights/days, how many nights/days did you get adequate sleep? 
How many hours of actual sleep did you usually get per day? (This may be different than the number of hours you spend in bed)

Many times a person's chronological age is not the same age that you feel that you are. We are interested to know your impression of your subjective age. Please indicate the age you FEEL in the drop down box below:

I FEEL as though I am years old

\begin{tabular}{|c|c|c|c|c|c|}
\hline \multirow{5}{*}{$\begin{array}{l}\text { Rate your level of physical discomfort } \\
\text { (pain, aching, stiffness, numbness, } \\
\text { tingling, burning, etc.) in each of the } \\
\text { following parts of your body over the past } \\
\underline{\mathbf{7} \text { days. }}\end{array}$} & \multicolumn{5}{|c|}{ Worst Pain Ever in your Life } \\
\hline & \multicolumn{5}{|c|}{ Severe Pain } \\
\hline & \multicolumn{3}{|c|}{ Moderate Pain } & & \\
\hline & \multicolumn{3}{|c|}{ Mild Pain } & & \\
\hline & \multicolumn{3}{|l|}{ No Pain } & & \\
\hline PN1. Neck & 1 & 2 & 3 & 4 & 5 \\
\hline PN2. Shoulders & 1 & 2 & 3 & 4 & 5 \\
\hline PN3. Upper Back & 1 & 2 & 3 & 4 & 5 \\
\hline PN4. Lower Back & 1 & 2 & 3 & 4 & 5 \\
\hline PN5. Arm/Elbows & 1 & 2 & 3 & 4 & 5 \\
\hline PN6. Wrist/Hands & 1 & 2 & 3 & 4 & 5 \\
\hline PN7. Hip/Thighs & 1 & 2 & 3 & 4 & 5 \\
\hline PN8. Knees & 1 & 2 & 3 & 4 & 5 \\
\hline PN9. Ankle/Feet & 1 & 2 & 3 & 4 & 5 \\
\hline
\end{tabular}

Please think back over the previous 7 days and answer the following questions about your health.

On how many days did you engage in aerobic or physical activity, such as walking, jogging, or cycling?

In the past 7 days, how many minutes did you engage in aerobic or physical activity on an average day?

In the past 7 days, on how many days did you smoke a cigarette?

On the days that you smoked, how many cigarettes did you typically smoke?

In the past 7 days, on how many days did you drink a caffeinated beverage? 
How many caffeinated beverages did you drink on an average day?

In the past 7 days, on how many days did you drink alcohol?

On a typical day, how many alcoholic drinks did you have?

In the past 7 days, what is the maximum number of alcoholic drinks you had on any given occasion?

Compared to other people your age, how would you rate your health?

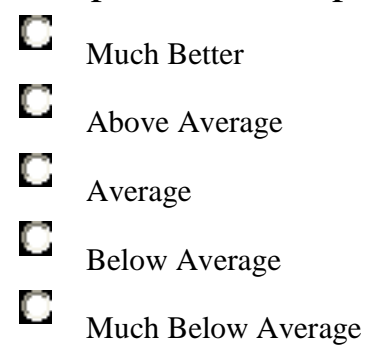

\begin{tabular}{|l|l|l|l|l|l|}
\hline $\begin{array}{l}\text { Using the scale, please indicate how much you agree or disagree with the } \\
\text { statement while keeping the past 7 days in mind. }\end{array}$ & $\begin{array}{l}\text { Strongly } \\
\text { Disagree }\end{array}$ & Disagree & Neutral & Agree & $\begin{array}{c}\text { Strongly } \\
\text { Agree }\end{array}$ \\
\hline $\begin{array}{l}\text { Overall, our unit was able to meet the } \\
\text { needs for nursing care this week. }\end{array}$ & & & & & \\
\hline $\begin{array}{l}\text { This week, my unit did a good job } \\
\text { applying available technology to patient } \\
\text { care needs. }\end{array}$ & & & & & \\
\hline $\begin{array}{l}\text { My unit functioned very well together as a } \\
\text { team this week. }\end{array}$ & & & & & \\
\hline $\begin{array}{l}\text { This week, my unit was very good at } \\
\text { responding to demanding situations. }\end{array}$ & & & & & \\
\hline $\begin{array}{l}\text { Given the severity of patients we nursed } \\
\text { this week, my unit's patients experienced } \\
\text { very good outcomes. }\end{array}$ & & & & & \\
\hline $\begin{array}{l}\text { I was satisfied with the quality of nursing } \\
\text { care I gave over the past week. }\end{array}$ & & & & & \\
\hline $\begin{array}{l}\text { Under the circumstances, I was happy } \\
\text { with the quality of care I provided over } \\
\text { the past 7 days. }\end{array}$ & & & & & \\
\hline $\begin{array}{l}\text { The patient care I gave this week met my } \\
\text { own standards for good patient care. }\end{array}$ & & & & & \\
\hline
\end{tabular}


Is there anything else that you would like to tell us about your job this week? 